\title{
Evolution of neuromodulation for lower urinary tract dysfunction
}

Citation for published version (APA):

Oerlemans, D. J. A. J. (2017). Evolution of neuromodulation for lower urinary tract dysfunction: past, present and future. [Doctoral Thesis, Maastricht University]. Maastricht University. https://doi.org/10.26481/dis.20170331do

Document status and date:

Published: 01/01/2017

DOI:

10.26481/dis.20170331do

Document Version:

Publisher's PDF, also known as Version of record

\section{Please check the document version of this publication:}

- A submitted manuscript is the version of the article upon submission and before peer-review. There can be important differences between the submitted version and the official published version of record.

People interested in the research are advised to contact the author for the final version of the publication, or visit the DOI to the publisher's website.

- The final author version and the galley proof are versions of the publication after peer review.

- The final published version features the final layout of the paper including the volume, issue and page numbers.

Link to publication

\footnotetext{
General rights rights.

- You may freely distribute the URL identifying the publication in the public portal. please follow below link for the End User Agreement:

www.umlib.nl/taverne-license

Take down policy

If you believe that this document breaches copyright please contact us at:

repository@maastrichtuniversity.nl

providing details and we will investigate your claim.
}

Copyright and moral rights for the publications made accessible in the public portal are retained by the authors and/or other copyright owners and it is a condition of accessing publications that users recognise and abide by the legal requirements associated with these

- Users may download and print one copy of any publication from the public portal for the purpose of private study or research.

- You may not further distribute the material or use it for any profit-making activity or commercial gain

If the publication is distributed under the terms of Article $25 \mathrm{fa}$ of the Dutch Copyright Act, indicated by the "Taverne" license above, 
Evolution of Neuromodulation

for Lower Urinary Tract Dysfunction

Past, Present and Future 
(C) Dennis Oerlemans, 2017

ISBN: 978-94-6233-559-2

Cover design \& typography: Patrick Ketelaars

Cover illustration: Draaideur by Hester Oerlemans

Print: Gildeprint

Publication of this thesis is financially supported by:

Stichting Wetenschappelijke Activiteiten Maastrichtse Urologie.

Allergan, Astellas, Cogentix Medical, Coloplast, Ferring Pharmaceuticals, Mayumana Healthcare, Olympus Nederland bv, Pohl Boskamp, Sanofi, Zambon.

No company has influenced the content of this thesis. 


\section{Evolution of Neuromodulation for Lower Urinary Tract Dysfunction}

\section{Past, Present and Future}

\section{Proefschrift}

ter verkrijging van de graad van doctor

aan de Universiteit Maastricht

op gezag van Rector Magnificus, prof. dr. Rianne M. Letschert volgens het besluit van het College van Decanen,

in het openbaar te verdedigen

op vrijdag 31 maart 2017 om 10.00 uur,

door

Dennis Johannes Adrianus Jozef Oerlemans 


\section{Promotores}

prof. dr. Ph.E.V.A. Van Kerrebroeck

prof. dr. G.A. van Koeveringe

Co-promotores

dr. E.H.J. Weil

dr. T.A.T. Marcelissen

\section{Beoordelingscommissie}

prof. dr. H.W.M. Steinbusch (voorzitter)

prof. dr. K. Everaert, Universiteit Gent, België

prof. dr. M. van Kleef

prof. dr. A.A.B. Lycklama à Nijeholt, Universiteit Leiden

prof. dr. A.A.M. Masclee 
Dokter, kunt u even komen

Breng uw beste medicijn

Ik heb er nu al jaren last vast

Ook al doet het niet echt pijn

Huub van der Lubbe (De Dijk), Groot Hart 



\section{CONTENTS}

10

Chapter 1

General Introduction and Aims of the Thesis

34 Chapter 2

Sacral Nerve Stimulation for Neuromodulation of the Lower

Urinary Tract

Neurourology and Urodynamics 27:28-33 (2008)

$58 \quad$ Chapter 3

Long Term Results of Neuromodulation by Sacral Nerve

Stimulation for Lower Urinary Tract Symptoms: a Retrospective Single Center Study

European Urology 49: 366-372 (2006)

8o Chapter 4

Medium-Term Experience of Sacral Neuromodulation by Tined

Lead Implantation

BJU International 99:107-110 (2006)

96 Chapter 5

Is On-Demand Sacral Neuromodulation in Patients With

OAB Syndrome a Feasible Therapy Regime?

Neurourology and Urodynamics 30:1493-1496 (2011)

114 Chapter 6

Sacral Neuromodulation in Patients with Idiopathic

Overactive Bladder after Initial Botulinum Toxin Therapy

The Journal of Urology 190: 2148-2152 (2013) 
132 Chapter 7

Sacral Neuromodulation in Patients with Combined

Micturition and Defecation Disorders

146 Chapter 8

General Discussion

175 Summary

179 Nederlandse samenvatting

183 Valorisation Addendum

189 Dankwoord

193 Lijst van publicaties

195 Curriculum Vitae

197 List of abbreviations

199 Thesis Defenses from MHeNs 

CHAPTER 1

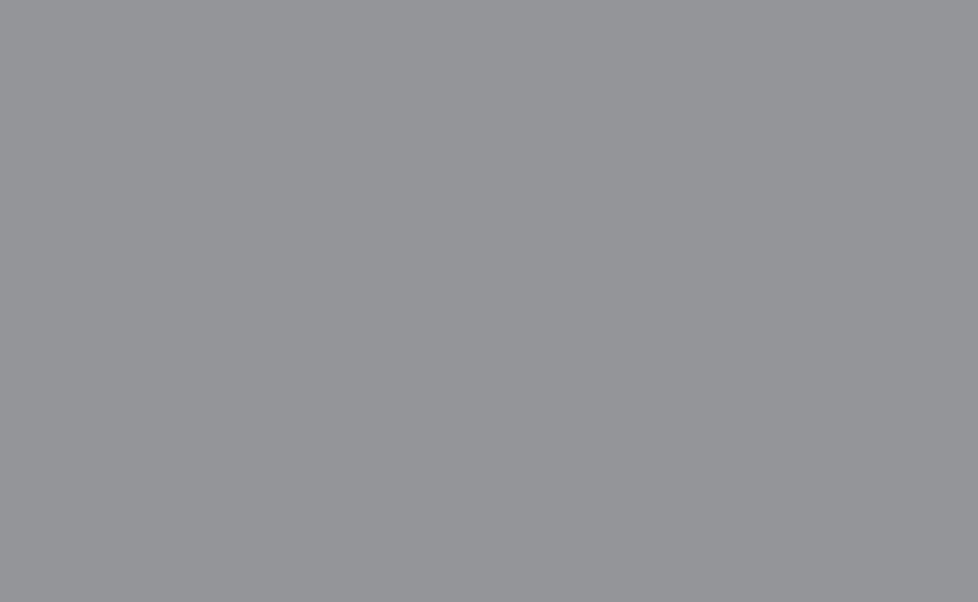


General Introduction and

Aims of the Thesis 


\section{$1 \cdot 1$}

\section{EVOLUTION OF NEUROMODULATION FOR LUTD (PAST, PRESENT, FUTURE)}

Chronic lower urinary tract dysfunction (LUTD) is a common disorder that has a significant impact on quality of life. The term 'dysfunction' refers to an abnormality in the physiology of the lower urinary tract, including the detrusor muscle, urinary sphincter, bladder neck and associated nervous system. According to the type of dysfunction, different clinical symptoms can arise. Overactive bladder syndrome $(\mathrm{OAB})$ is the most common disorder, and is defined as the presence of urinary urgency, usually accompanied by frequency and can occur with or without urinary incontinence. In contrast, LUTD can also result in the inability to evacuate urine. Hypocontractility of the detrusor muscle or hypertonicity of the urinary sphincter can cause chronic (non-obstructive) urinary retention.

Initial management consists of behavioural therapy. This includes bladder training, bladder control strategies, pelvic floor muscle training and fluid management. Behavioural therapy can target bladder function by modifying voiding habits with bladder training and delayed voiding, or target the bladder outlet with pelvic floor muscle strengthening or relaxation techniques, depending on the type of disorder. Patients with detrusor hypocontractility are usually managed with clean intermittent self catheterization.

Second line treatment for OAB consists of oral drug therapy. The use of antimuscarinic medications has been thoroughly studied and is considered safe and effective in the treatment of OAB with or without urinary incontinence. There are multiple antimuscarinics available and all have similar efficacies, but differ in their side-effect profiles. In general, antimuscarinics are frequently discontinued due to bothersome side-effects. A review of the literature demonstrated that $43-83 \%$ of women abandon antimuscarinic therapy within 1 month, and less than $35 \%$ of women continue their medication after the first year. ${ }^{1}$ Recently, the US Food and Drug Administration (FDA) approved the use of beta-3 agonist mirabegron as a new drug for OAB. The long-term effectiveness and tolerability of this drug still needs to be evaluated. 
A considerable amount of patients do not respond to first or second-line treatment due to insufficient response or intolerability. Treatment of these patients has long been a therapeutic challenge since there were no valid options except intrusive surgery, such as bladder augmentation or urinary diversion. Hence there was a need for less invasive procedures. Instead of focusing on the bladder and urethral muscle as a target for treatment, methods were investigated to modulate the neural pathways of micturition. Experiments with different ways of electrical stimulation led to the development of neuromodulation therapies that are nowadays implemented in the standard care of patients with LUT disorders. In the last two decades, various minimally-invasive treatments have become available, which have closed the gap between conservative and invasive treatment. In the early 9o's, sacral neuromodulation (SNM) was introduced as a treatment for patients with OAB. In this treatment, an electrical signal is applied to the sacral nerve roots S1-3 with an implantable electrode. This treatment proved to be beneficial for patients with overactive bladder as well as chronic urinary retention. At the same time, percutaneous and transcutaneous techniques were developed for the same purpose. Furthermore, chemical methods of neuromodulation have also arisen, in particular intravesical injections with botulinum toxin-A. Nowadays, these neuromodulatory techniques have achieved a prominent role in the treatment of patients with LUTD. Figure 1 illustrates their current position in the clinical treatment of OAB (EAU guidelines 2012). ${ }^{2}$

This thesis focuses on the evolution of neuromodulation in the treatment of LUTD, evaluating advancements in technique, patient selection and expanding indications. 


\section{FIGURE 1}

Treatment algorithm for women with urinary incontinence

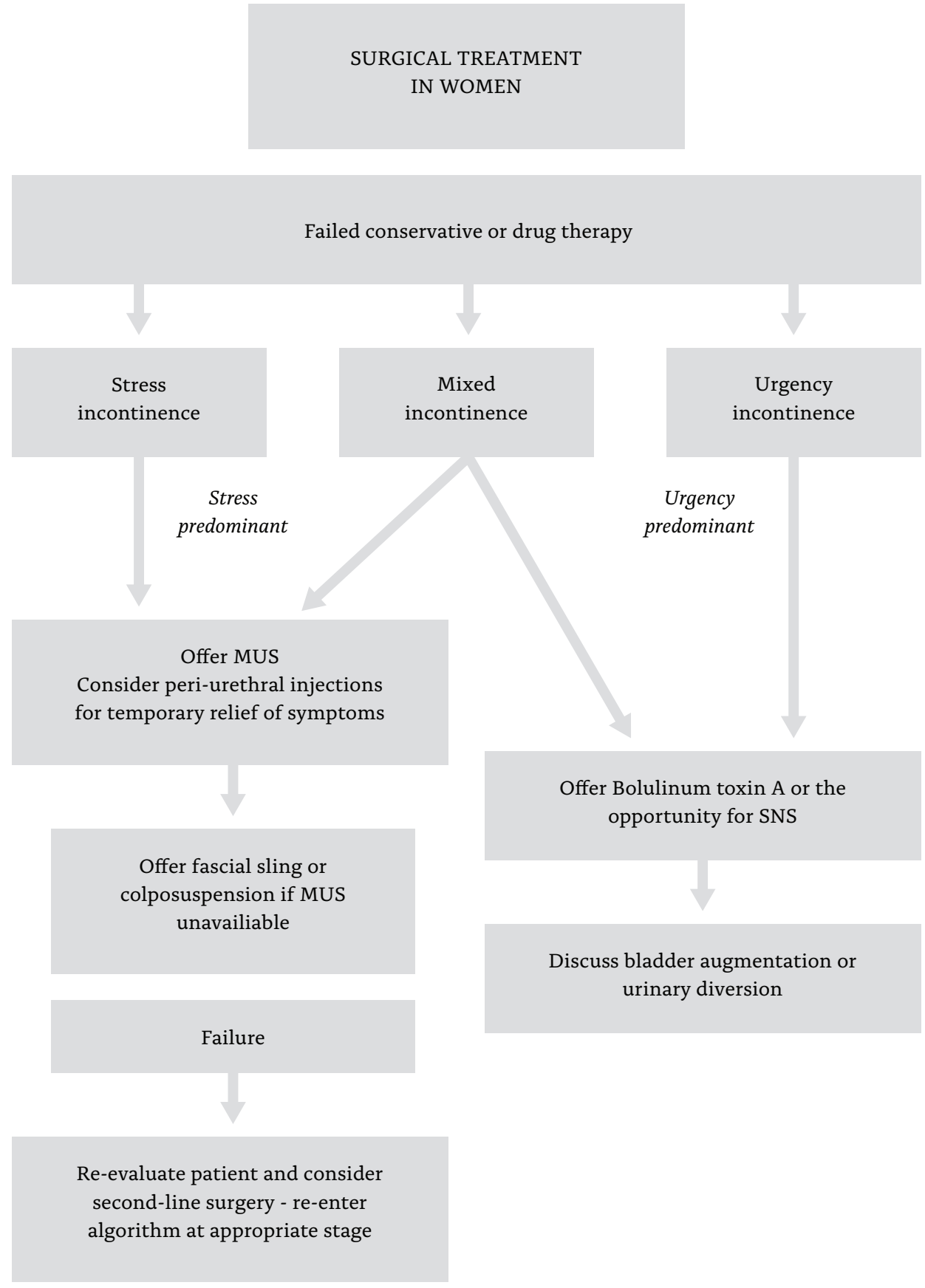




\section{2 \\ WHAT IS NEUROMODULATION?}

Neuromodulation is the physiologic principle that activity in one neural pathway modulates the pre-existing activity in another through synaptic interaction. During the micturition process, different neural pathways are involved to ensure correct interaction between muscle contraction and relaxation. Micturition includes two phases: urinary storage and elimination. During the storage phase, the bladder works as a reservoir and the urethra remains closed avoiding any urine leakage, while in the elimination phase, the bladder works as a pump that expels urine at the same time as the urethra opens, allowing a free conduct for urine flow. A good coordination of the antagonistic functions of the bladder and urethra demand a complex interaction between the peripheral and central nervous system. The main goal of neuromodulation is to restore the normal micturition reflexes that are involved in lower urinary tract (LUT) functioning. The neural control of the LUT involves three sets of peripheral nerves: the autonomic (parasympathetic, sympathetic) and the somatic nerves. Although dependent on autonomic reflexes, vesical and urethral functions are controlled by higher cortical centers to ensure the voluntary control of micturition. This feature differentiates these organs from other viscera innervated by the autonomic nervous system. The pelvic nerve is the main (parasympathetic) nerve responsible for bladder contraction in the elimination phase and it uses the neurotransmitter acetylcholine in its synapses. The hypogastric (sympathetic) nerve is originated in the thoracolumbar spinal cord nucleus (T10-L2), which is responsible for conducting the impulses resulting in urethral smooth muscle contraction in the storage phase. An inhibitory stimulus is sent to the detrusor smooth muscle and excitatory stimulus to the urethra. The somatic control is mediated by the pudendal nerve and the efferent motorneurons originate in the somatic nucleus in the sacral spinal cord (S2-S4), commonly referred to as Onuf's nucleus. Pudendal nerve stimuli cause (voluntary) contraction of the external urethral sphincter and pelvic muscles in the urinary storage phase.

Although the pathophysiology of LUT dysfunction is not well understood, 
multiple changes on different levels (e.g. detrusor, urethra, neural innervation) are involved. Continence is maintained by a synergic accommodation of the smooth muscle of the bladder wall to increasing volumes of urine without a change in detrusor pressure and closure of the bladder outlet. An involuntary contraction of the detrusor (as seen in detrusor overactivity) can lead to leakage of urine. Alternatively, insufficient contraction of the detrusor (detrusor underactivity) can lead to difficult elimination or urinary retention. It is hypothesized that by modulation of the disbalanced neural activity (either over- or underactivity), normal micturition can be restored.

\section{FIGURE 2}

Neuronal innervation of the lower urinary tract

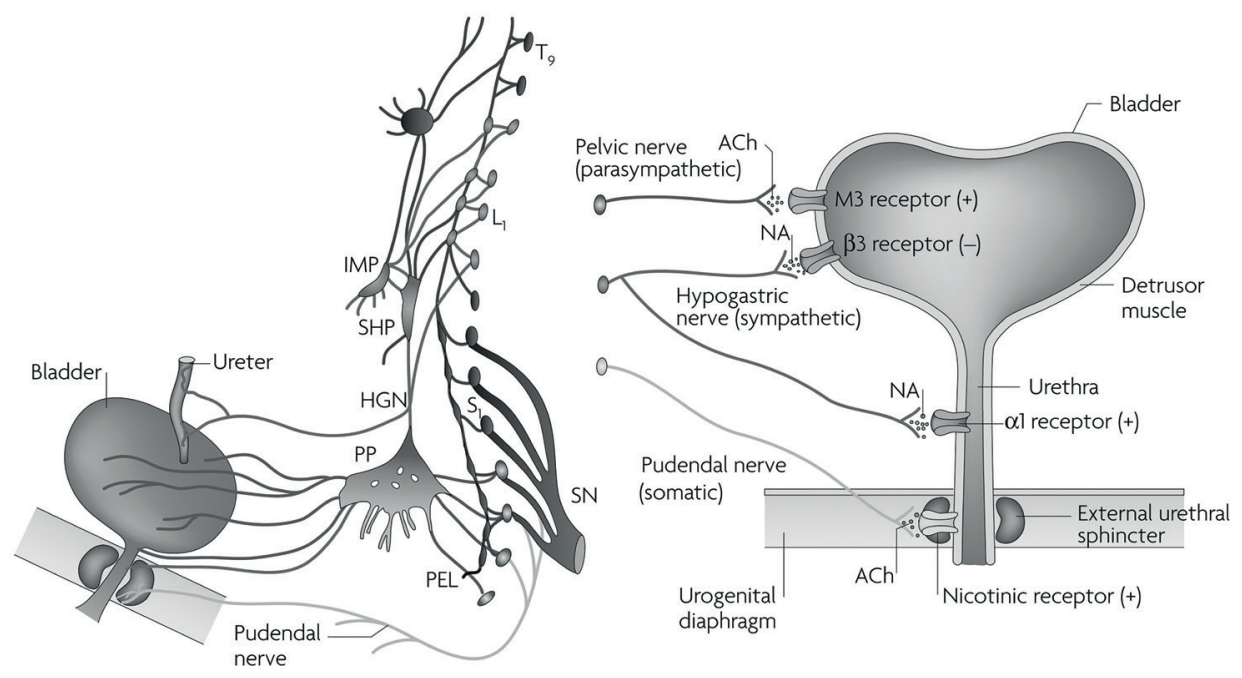

Fowler CJ, Griffiths D, de Groat WC. The neural control of micturition. Nat Rev Neurosci 20o8;9:453-66. 


\section{$1 \cdot 3$ \\ HOW DOES NEUROMODULATION WORK?}

Electrical stimulation of an organ can be done in different ways. A nerve or muscle can be stimulated directly or indirectly. In neurostimulation nerves or muscles are directly stimulated by efferent neurons to achieve immediate responses (e.g. muscle contraction). In neuromodulation electrical stimuli are applied to alter present neurotransmission processes by afferent neurons. Initially it was hypothesized that stimulation of the $S_{3}$ level amplified the continence reflex by stimulation of the pelvic nerve. A support for this theory was the contraction of perianal muscles during acute stimulation of the $\mathrm{S}_{3}$ roots. ${ }^{3}$ Fowler et al in contrast registered increased latencies with EMG of the anal sphincter on stimulation of the $\mathrm{S} 3$ root, compared to stimulation of the pudendal nerve, suggesting that SNM acts through a polysynaptic reflex. No distinction between a spinal or a supraspinal reflex was found. ${ }^{4}$ Schurch confirmed the involvement of afferent pathways and spinal reflexes by latency measurements in patients with complete spinal cord injury. ${ }^{5}$ However, SNM did not improve voiding in these patients and it was suggested that supraspinal mechanisms are also involved. SNM might act merely on the processing of the sensory information from the bladder and has an influence on the pathological transmission of afferent information from the bladder through the C-fibers. ${ }^{6}$ Furthermore, recent f-MRI images indicate that afferent information in the periaqueductal grey and in the cingulate cortex can be normalized with SNM?

\section{$O A B$}

In patients diagnosed with overactive bladder, SNM inhibits detrusor activity without affecting urethral resistance or the strength of detrusor contractions during voiding. ${ }^{8}$

Sievert et al showed that bilateral SNM in patients with a complete spinal cord injury initiated during the spinal shock phase could prevent the development of detrusor overactivity. ${ }^{9}$ This could be an indication for a modulatory effect at the level of the spinal cord itself. Studies by Blok and by Dasgupta showed 
activity of centers in the brain involved in micturition. SNM can potentially enhance or reduce the activity in these centers suggesting a central effect. ${ }^{8,10}$ Blok studied patients with chronic stimulation and compared them to patients with a new implanted system that was first activated (acute SNM) in a PET scanner. He showed that areas in the brain previously implicated in detrusor overactivity were influenced by SNM. During acute SNM, areas involved in sensorimotor learning were involved in change of activity.

\section{Urinary Retention}

Functional (non-obstructive) causes of urinary retention are a decreased or absent tonus of the detrusor muscle, or hypertonia of the sphincter. In patients with Fowler'syndrome, an abnormal electromyographic activity of the external urethral sphincter is seen in combination with polycystic ovaries." Dasgupta compared healthy controls with Fowler's syndrome patients. During PET scanning, activity was seen in the brain stem and limbic cortical regions in the healthy controls, whereas in the patients with Fowler's syndrome, this activity was absent with a full bladder. This activity could be restored by SNM. Functional MRI was also used to measure brain responses to a full bladder in patients with Fowler's syndrome, and compared to a situation with an empty bladder. These authors concluded that an overactive urethra generates abnormally strong inhibitory afferent signals, so effectively blocking bladder afferent activity at the sacral level and deactivating the periaqueductal grey and higher centres, with consequent loss of bladder sensation and ability to void. SNM has been postulated to suppress the guarding reflex, and as a consequence, decreased urethral sphincter tone facilitates voiding.?

The exact working mechanism of SNM in treating LUTD is complex and still not fully understood. It probably involves different modes of action and acts on various levels of the nervous system, including sacral, spinal, supraspinal and cortical areas of the brain. 


\section{4 \\ DEVELOPMENT OF NEUROMODULATION}

In 1878 Saxtorph reported (transurethral) intravesical stimulation in patients with hypocontractile bladder and urinary retention. ${ }^{12}$ In 1958 Katona's group started transurethral electrical stimulation, a technique derived from Saxtorph. Their hypothesis was that stimulation of intramural bladder receptors in patients with incomplete central or peripheral nerve lesions may reactivate these receptors. In 1975 they published results of 420 patients treated with this therapy. Of these patients, 314 achieved micturition control. ${ }^{13}$

McGuire performed direct bladder stimulation in dogs in 1954. Boyce et al found that two or more bipolar wire electrodes produced a more uniform response than a single lead and stimulated a group of 3 paraplegic men directly in the bladder musculature. One of these male subjects had a successful response and another had partial success. ${ }^{14}$ New electrodes and techniques were developed over the years, but results remained disappointing. In 1978 Jonas reported on eleven patients, of whom only one was successfully treated with this therapy. ${ }^{15}$ Other groups studied stimulation of pelvic nerves, but pelvic nerves were thought not to tolerate stimulation for long periods of time. ${ }^{16}$

During the 196o's various implantable pelvic floor stimulators were developed. There was a high incidence in technical failure and electrode dislocation. ${ }^{17}$ At the same time experiments started with spinal cord stimulation in animal models. Nashold et al reported the first results in humans in $1972 .{ }^{18}$ Jonas and Tanagho showed that muscle response of the bladder during stimulation in patients with spinal cord transection rose and dropped more slowly than that of the urethral sphincter. After the stimulus there was a short time when bladder pressure exceeded sphincteric pressure, and thus repetitive stimuli resulted in complete bladder evacuation. ${ }^{19}$

Later, Brindley developed an electrode for long term stimulation and in 1978 the first patients were implanted with this prosthesis for direct sacral nerve stimulation. ${ }^{20}$ Tanagho and Schmidt demonstrated that stimulation of the sacral root $\mathrm{S} 3$ modulates detrusor and sphincter action and could be used in 
daily practice in patients with lower urinary tract dysfunction. ${ }^{21}$ This technique was further improved and in 1997 the FDA in the United States approved SNM for therapeutic use in urgency urinary incontinence. 


\section{5 \\ NEUROMODULATION TECHNIQUES}

Sacral Neuromodulation (SNM)

In order to achieve SNM a sacral root is stimulated with a quadripolar lead positioned in the sacral foramen (in general $\mathrm{S}_{3}$ ). The lead is connected to an implantable pulse generator which is located in a subcutaneous pocket, mostly in the buttock area. Since 1999, a percutaneous nerve evaluation (PNE) has been used to monitor the effect of SNM prior to implantation. During the PNE, a wire electrode is placed in a sacral foramen and connected to an external stimulator. The test duration is limited to $3-7$ days due to the high risk of lead migration. Improvement of symptoms is monitored during the test with voiding diaries. An improvement of at least $50 \%$ compared to baseline is defined as success. After a successful test period a permanent IPG can be implanted. Later, the twostage procedure was introduced by Janknegt et al. Patients who experienced an initial good effect of PNE (but loss of this effect after one or two days) were tested again with a definitive lead implanted and connected to an external stimulator (first stage). If this showed a positive effect, a definitive device was implanted at a second stage. ${ }^{22}$

In 2002 a self-anchoring lead was introduced by Spinelli et al, which allowed a percutaneous placement of the lead, the so-called Tined Lead Procedure (TLP). Originally, the permanent lead was implanted under direct vision using an open surgical procedure with a midline incision over the sacrum. Advantages of the percutaneous lead placement are a shorter operation time and less invasiveness of the procedure. Furthermore, the lead can be introduced under local anesthesia so that sensory responses can be evaluated during the procedure. ${ }^{23}$ After the implantation of the tined lead, a test phase can be performed for a prolonged period of time (up to 4 weeks) in the same way as the original two-stage procedure.

\section{TENS}

Transcutaneous electrical nerve stimulation (TENS) applies a small electrical current to the skin that can usually be felt and stimulates sensory nerves. TENS 
is often used in patients with chronic pain syndromes. In urology it was first reported to have had positive results in patients with interstitial cystitis. It relieved not only pain, but patients also reported less frequency. ${ }^{24} \mathrm{~A}$ disadvantage of TENS is the time-consuming effect. TENS is applied for 30 minutes a day for 60 days. Almost all published studies report only short term results.

\section{Vaginal stimulation}

As mentioned earlier, different ways of electrical stimulation have been tested and used over the past few decades. Uncontrolled studies using vaginal and also rectal electrical stimulation reported success rates of up to $80 \%{ }^{25}$ Many variables such as duration of therapy and electrical parameters differ. Spruijt et al. concluded after a randomized feasibility study to treat incontinent elderly women, that vaginal stimulation of the pelvic floor has a high physical and emotional cost for the individual. The effectiveness is low and it is not reasonable to advise elderly women with urinary incontinence to undertake this treatment procedure. Their study was terminated because of the negative outcome. ${ }^{26}$ Compared to SNM, this therapy is time-consuming, less effective and physically and emotionally more invasive.

\section{PTNS}

Percutaneous tibial nerve stimulation (PTNS) is a peripheral neuromodulation technique. The nervus tibialis posterior is stimulated above the median malleolus of the tibia. PTNS uses an acupuncture point that originates from Chinese medicine. The possible working mechanism is neuromodulation at the spinal level. ${ }^{27}$ PTNS requires repeated sessions of stimulation. In patients with OAB for example, 8 weekly sessions are performed. PTNS is used in patients with OAB, urinary incontinence, fecal incontinence and in patients with chronic pelvic pain. A review by Biemans et al reported an improvement in incontinence episodes, frequency and urgency of $60-80 \%$ in patients with OAB. Furthermore, they concluded that only minimal evidence is provided for the efficacy of PTNS in fecal incontinence and chronic pelvic pain. The CONFIDeNT trail (double-blind, multicentre, pragmatic, parallel-group, randomised controlled trial) studied 227 patients, 115 patients treated with PTNS, 112 patients treated with a sham therapy. They concluded PTNS given for 12 weeks does not confer significant clinical benefit over sham electrical stimulation in the treatment of adults with faecal incontinence. ${ }^{28}$

PTNS is considered a safe intervention. ${ }^{29}$ A recent review that compared PTNS to SNM reported good results for both in OAB patients. SNM is the only 
treatment that has shown efficacy in UR. Regarding cost-effectiveness, after a period of 2 years SNM began to show improved effectiveness and favorable cost value compared to PTNS. This study concluded that PTNS is an office-based procedure that provides a carryover clinical effect. SNM on the contrary offers constant stimulation without the burden of weekly or monthly visits. ${ }^{30}$

\section{Pudendal Nerve Stimulation}

The pudendal nerve is one of the major nerves that innervates the pelvic floor, the external and urethral sphincters and the pelvic organs. In 1990 Schmidt et al reported on two patients with myelomeningocele implanted with chronic pudendal stimulation (PS). ${ }^{31}$ Patients not responding to SNM were the main reason for Spinelli et al to study a less invasive procedure to stimulate the pudendal nerve. ${ }^{32}$ Most patients tested can be helped with SNM (60-70\%), those who do not respond to SNM seem to be good candidates for a test stimulation with PS. Peters et al. retrospectively studied a group of patients having a tined lead placed at the pudendal nerve via the ischial-rectal approach. The majority of 84 patients had interstitial cystitis/painful bladder syndrome, or overactive bladder. Pudendal response occurred in 6o/84 (71.4\%). Almost all (93.2\%) who had previously failed sacral neuromodulation responded to pudendal stimulation. Outcomes were evaluated in 55 continuing on PS (median follow up 24.1 months). Over time, significant improvements in frequency $(\mathrm{P}<0.0001)$, voided volume $(\mathrm{P}<0.0001)$, incontinence $(\mathrm{P}<0.0001)$, and urgency $(\mathrm{P}=0.0019)$ occurred. ICSI-PI scores significantly improved over 12 months $(\mathrm{P}<0.0001)$. Survey responses indicated that most still had a device (35/40; 87.5\%) continuously in use $(24 / 29 ; 82.8 \%)$, and overall bladder, pelvic pain, incontinence, urgency, and frequency symptoms had improved. They concluded that PS is a reasonable alternative in complex patients refractory to other therapies including sacral neuromodulation. ${ }^{3}$ 


\section{6}

\section{CLINICAL APPLICATIONS}

The lower urinary tract, anorectal channel and pelvic floor are closely related, both anatomically and functionally. Dysfunction of the lower urinary tract and dysfunction of the bowel are problems that can have a major impact on the quality of life. Patients may complain about multiple symptoms: urgency and frequency, urgency urinary incontinence, urinary retention, genito-urinary pain syndromes and also fecal incontinence and constipation.

$O A B$

Various forms of neuromodulation have proven to be effective in the treatment of OAB. In a worldwide clinical study, Van Voskuilen et al described SNM success rates of $58 \%$ in leaking episodes, to a $68 \%$ success rate in patients with heavy leaking episodes five years after implantation. At a 1 year follow-up, the average number of voids had decreased from $19.3 \pm 7$ to $13 \pm 7.9$. This reduction was maintained through to the long-term follow-up after 5 years. ${ }^{34}$

A systematic review by Siddiqui reported efficacy results of 67 to $84 \%$ based on 5 clinical studies. They concluded that SNM results in significantly less pad use and fewer incontinent episodes per day in women with refractory OAB. Almost half of the subjects reported no daily incontinence episodes after treatment with SNM, and results were maintained up to 5 years after implantation. ${ }^{35}$

Very recently the results of a prospective, randomized, multicenter study evaluating SNM compared to standard medical therapy (SMT) were published. In total, 147 subjects were randomized (70 to SNM and 77 to SMT). The primary intent to treat analysis showed OAB therapeutic success was significantly greater in the SNM group (61\%) than the SMT group (42\%; P=0.02). In the as treated analysis, OAB therapeutic success was $76 \%$ for SNM and $49 \%$ for SMT $(\mathrm{P}=0.002){ }^{36}$

A paper by Guo et al. reported on the results of TENS in post-stroke patients with urinary incontinence. Patients in the treatment group were treated daily for 30 minutes for a period of 60 days. TENS improved incontinence symptoms 
and enhanced quality of life compared to the control group..$^{37}$

A review by Gaziev et al found an overall percentage of patients classified as successfully treated for OAB symptoms with PTNS of $54 \cdot 5-79.5 \%$. The definition of success differs among studies from the use of urodynamic data to clinical parameters and quality of life measures. ${ }^{38}$

As mentioned earlier, Spinelli et al performed pudendal stimulation in patients that did not respond to SNM. A perineal approach in 6 patients and a posterior approach in 9 was studied. All were neurogenic patients. The average number of incontinent episodes decreased from 7 to 2.6 episodes, eight patients became continent. Four out of seven patients with associated constipation at baseline reported normalization of bowel evacuation..$^{32}$

\section{Urinary Retention}

Most patients with functional urinary retention perform clean intermittent catheterization (CIC) for bladder emptying. SNM can restore micturition in patients with non-obstructive urinary retention. An average decrease in the number of catheterizations in the group treated with SNM was seen from $5.3 \pm 2.8$ at baseline to $1.9 \pm 2.8$, with a clinical efficacy of $58 \%$ five years after implantation. ${ }^{34}$

Results from a 10-year experiment in this group treated with SNM showed a success rate of $72 \%$ (43 out of 60 patients voided spontaneously after implantation) and $50 \%$ of patients no longer needed to use CIC. Women with a normal urethral sphincter electromyogram had worse outcomes than women with an abnormal test (43\% vs $76 \%$ ). ${ }^{39}$

PTNS is also proposed as a treatment option for these patients. Vandoninck et al published one of the few studies with results for this group. The primary outcome measure was a reduction of the total catheterized volume per 24 hours. Using a reduction of $>50 \%$, the percentage of responders was $41 \% .4^{\circ}$

\section{Bowel Dysfunction}

Fecal incontinence (FI) is a common symptom that can have a devastating impact on the quality of life. Reported prevalence ranges from $7-15 \%$ in community dwelling men and women. ${ }^{41}$ Because healthcare providers do not often screen for these symptoms and patients do not voluntarily report them, the real problem is probably underreported. Constipation presumably has less impact on the quality of daily life; however it represents a significant burden and is often associated with FI or urinary problems. A recent survey among Brazilian women with pelvic floor dysfunction reported a prevalence of $67 \%$ for constipation in a group of women with defecation disorders. Of the women with UI $23 \%$ had 
associated FI. ${ }^{42}$ Both of these conditions can be treated with SNM. A review by Thin et al reported success rates of $63 \%$ (short term) to $54 \%$ (long term) for the treatment of fecal incontinence. ${ }^{43}$ Thomas et al presented a review on the use of SNM in patients with constipation. Test stimulation was successful in 42-100 per cent of patients. In those who proceeded to permanent SNM, up to 87 per cent showed an improvement in symptoms at a median follow-up of 28 months. The success of stimulation varied depending on the outcome measure being used. 44

In patients with fecal incontinence PS was also tested. A pilot study showed $>50 \%$ short-term improvement in $50 \%(5 / 10)$ of the tested patients. These patients were implanted and at a median follow-up time of 24 months, three of the patients had maintained these results. ${ }^{45}$

Edenfield et al recently published a review on the effect of PTNS in patients with fecal incontinence. They found 13 case series and 4 randomized controlled trials. In total, 745 subjects were studied, and of those, $90 \%$ were women and $10 \%$ were men. Studies involved PTNS in $57 \%(428 / 745)$ of the subjects, transcutaneous electrical nerve stimulation in $30 \%(223 / 745)$, and sham technique in $13 \%$ (94/745). Therapy frequency, maintenance therapy, and follow-up time varied across the studies. Eleven studies assessed FI episodes and bowel movement deferment time; all but 1 showed statistical improvement after therapy. They concluded that multiple low-quality studies show improvement in FI after PTNS. ${ }^{46}$ These promising results could not be confirmed in the CONFIDeNT trial, a double-blind, multicentre, pragmatic, parallel-group, randomized controlled trial. They had to conclude that PTNS given for a period of 12 weeks did not confer significant clinical benefit over sham electrical stimulation in the treatment of adults with fecal incontinence. ${ }^{28}$

\section{Chronic Pelvic Pain}

Urological pain syndromes like interstitial cystitis and chronic pelvic pain syndrome or non-bacterial prostatitis can be treated with medication, analgetics, physiotherapy and sometimes dietary manipulation. If these therapies are not effective, other (invasive) therapies including SNM can be considered. ${ }^{47}$

Marcelissen et al evaluated the role of SNM in the treatment of chronic pelvic pain and identified 12 papers on this subject. Of these papers, 10 mainly addressed the efficacy of SNM in patients with interstitial cystitis/bladder pain. The percentage of patients who responded to test stimulation was reported to be between $51 \%$ and $77 \%$. Of the 10 articles, 7 reported on treatment outcome after implantation. The duration of follow-up ranged between 5 and 87 months. 
The mean reduction in pain scores was reported to be between $40 \%$ and $72 \% 48$. Although these results seem promising, the evidence of SNM in the treatment of pelvic pain is still limited due to the lack of high-quality trials and long-term follow-up.

The efficacy of PTNS in the treatment of chronic pelvic pain was evaluated in one study. A total of 89 patients with therapy-resistant pelvic pain were randomized to receive either PTNS $(n=45)$ or sham treatment $(n=44)$. An objective response was observed with the VAS and NIH-CPSI scores after 12 weeks of PTNS in 18 (40\%) and 30 (66.6\%) of the patients. The mean NIH-CPSI scores did not differ significantly in the sham treatment group. ${ }^{49}$ 


\section{7}

\section{OUTLINE OF THIS THESIS}

This thesis focuses on the evolution of SNM and further differentiation of this technique.

Chapter 1 contains the introduction, in which the working mechanism, techniques and clinical applications of neuromodulation are discussed.

Chapter 2 presents an overview of the clinical results published on SNM, including aspects of patient selection, efficacy and adverse events of this treatment.

Chapter 3 presents the long-term results of a transversal cohort study on 149 SNM implants performed in our center between 1990 and 2003. In this group of 149 patients, 20 patients had a neurological cause for their urological complaints. In 129 patients re-operations were performed, but no severe adverse events were seen.

Chapter 4 reports on our experience with 49 tined lead implants. This new technique changed the treatment algorithm and results. With a mean follow-up of 15.5 months, $90.3 \%$ of the patients maintained more than $50 \%$ improvement in at least one of the relevant voiding diary parameters. One of the reasons for re-intervention is IPG battery depletion. With the introduction of the Interstim II with a smaller battery capacity, we studied the feasibility of on-demand SNM use in patients with OAB syndrome (Chapter 5 ).

Due to the upcoming use of intravesical botulin toxin for OAB syndrome, many of our patients were treated with this therapy before a test stimulation with SNM was planned. Chapter 6 explores whether SNM is still effective in this patient group.

Numerous reports have been published on the results of SNM on purely urinary or fecal incontinence. However, results of patients with double incontinence are rarely published. We performed a study on patients with double incontinence (fecal and urinary) treated with SNM. The results and predictive factors are described in Chapter 7.

Chapters 8 and 9 conclude this thesis with a discussion and an overview on the topics of this thesis, and also present future perspectives of the SNM technique. 


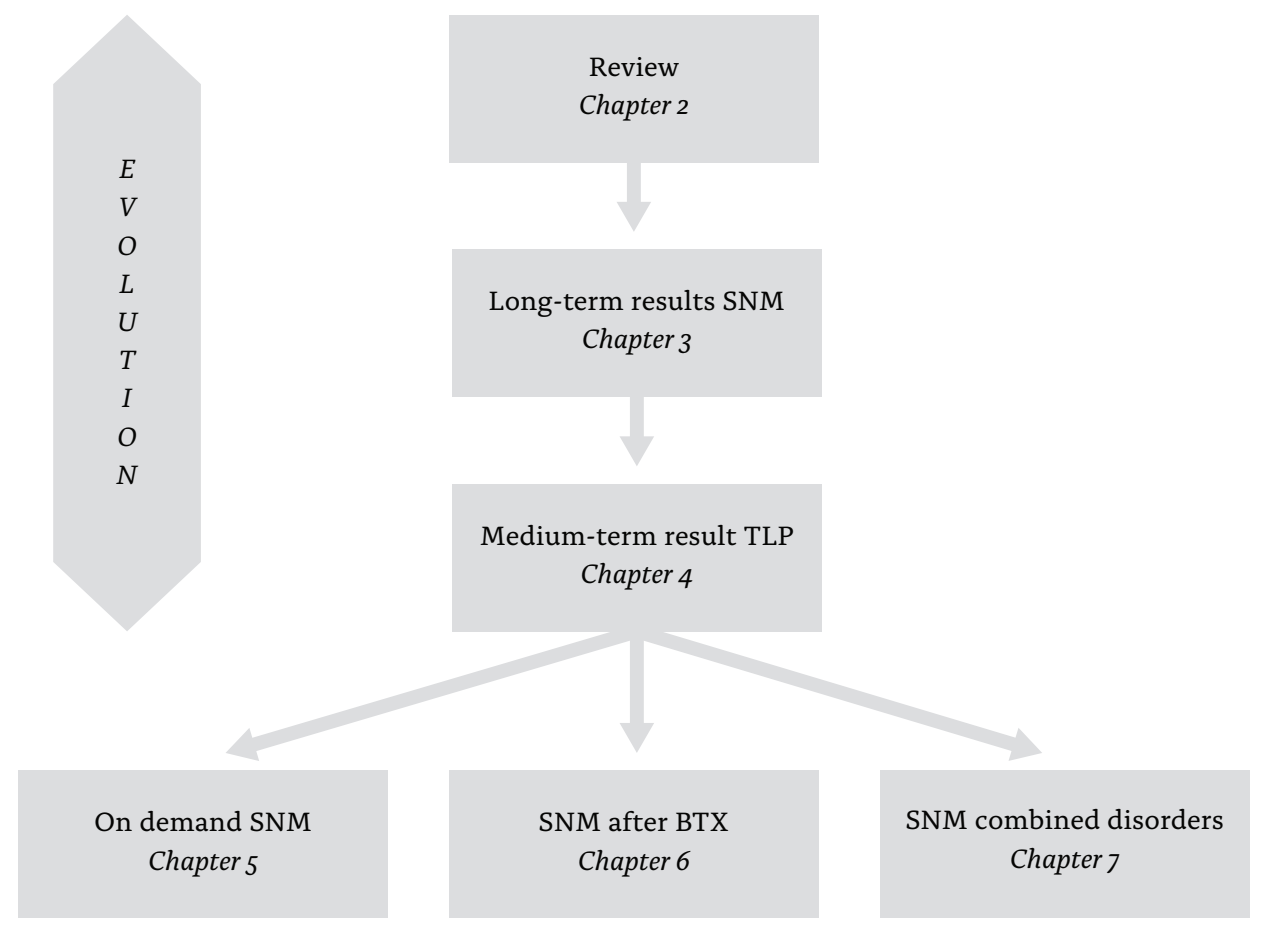




\section{REFERENCES}

1 Sexton CC, Notte SM, Maroulis C, et al. Persistence and adherence in the treatment of overactive bladder syndrome with anticholinergic therapy: a systematic review of the literature. Int J Clin Pract, 2011. 65:567-85.

2 Lucas MG, Bosch RJ, Burkhard FC, et al. EAU guidelines on surgical treatment of urinary incontinence. Eur Urol, 2012. 62:1118-29.

3 Lindstrom S, Fall M, Carlsson CA, Erlandson BE. The neurophysiological basis of bladder inhibition in response to intravaginal electrical stimulation. J Urol, 1983. 129:405-10.

4 Fowler CJ, Swinn MJ, Goodwin RJ, Oliver S, Craggs M. Studies of the latency of pelvic floor contraction during peripheral nerve evaluation show that the muscle response is reflexly mediated. J Urol, 2000. 163:881-3.

5 Schurch B, Reilly I, Reitz A, Curt A. Electrophysiological recordings during the peripheral nerve evaluation (PNE) test in complete spinal cord injury patients. World J Urol, 2003. 20:319-22.

6 Shaker H, Wang Y, Loung D, Balbaa L, Fehlings MG, Hassouna MM. Role of C-afferent fibres in the mechanism of action of sacral nerve root neuromodulation in chronic spinal cord injury. BJU Int, 2000. 85:905-10.

7 Kavia R, Dasgupta R, Critchley H, Fowler C, Griffiths D. A functional magnetic resonance imaging study of the effect of sacral neuromodulation on brain responses in women with Fowler's syndrome. BJU Int, 2010. 105:366-72.

8 Blok BF, Groen J, Bosch JL, Veltman DJ, Lammertsma AA. Different brain effects during chronic and acute sacral neuromodulation in urge incontinent patients with implanted neurostimulators. BJU Int, 2006. 98:1238-43.

9 Sievert KD, Amend B, Gakis G, et al. Early sacral neuromodulation prevents urinary incontinence after complete spinal cord injury. Ann Neurol, 2010. 67:74-84.

10 Dasgupta R, Critchley HD, Dolan RJ, Fowler CJ. Changes in brain activity following sacral neuromodulation for urinary retention. J Urol, 2005. 174:2268-72.

11 Fowler CJ, Christmas TJ, Chapple CR, Parkhouse HF, Kirby RS, Jacobs HS. Abnormal electromyographic activity of the urethral sphincter, voiding dysfunction, and polycystic ovaries: a new syndrome? BMJ, 1988. 297:1436-1438.

12 Saxtorph M. Strictura urethrae-Fistula perinei-Retentio urinae. Clinisk Chirurgi. 1878, Gyldendalske Forlag p. 265.

13 Katona F. Stages of vegatative afferentation in reorganization of bladder control during intravesical electrotherapy. Urol Int, 1975 · 30:192-203.

14 Boyce WH, Lathem JE, Hunt LD. Research Related to the Development of an Artificial Electrical Stimulator for the Paralyzed Human Bladder: A Review. J Urol, 1964. 91:41-51.

15 Jonas U, Hohenfellner R. Late results of bladder stimulation in 11 patients: followup to 4 years. J Urol, 1978. 120:565-8. 
16 Tanagho EA. Induced micturition via intraspinal sacral root stimulation: clinical implications In: Hambrecht FT, Reswick JB, eds. Biomedical engineering and instrumentation series, F.T. Hambrecht and J.B. Reswick, Editors. 1977, Marcel Dekker: New York. p. 157-71

17 Caldwell KP, Flack FC, Broad AF. Urinary Incontinence Following Spinal Injury Treated by Electronic Implant. Lancet, 1965. 1:846-7.

18 Nashold BS, Jr., Friedman H, Glenn JF, Grimes JH, Barry WF, Avery R. Electromicturition in paraplegia. Implantation of a spinal neuroprosthesis. Arch Surg, 1972. 104:195-202.

19 Jonas U, Tanagho EA. Studies on the feasibility of urinary bladder evacuation by direct spinal cord stimulation. II. Poststimulus voiding: a way to overcome outflow resistance. Invest Urol, 1975. 13:151-3

20 Brindley G, Polkey C, Rushton D. Sacral anterior root stimulators for bladder control in paraplegia. Paraplegia, 1982. 20:365-381.

21 Tanagho EA, Schmidt RA. Bladder pacemaker: scientific basis and clinical future. Urology, 1982. 20:614-9.

22 Janknegt RA, Weil EH, Eerdmans PHA. Improving neuromodulation technique for refractory voiding dysfunctions: two-stage implant. Urology, 1997. 49:358-362.

23 Spinelli M, Giardiello G, Gerber M, Arduini A, van den Hombergh U, Malaguti S. New sacral neuromodulation lead for percutaneous implantation using local anesthesia: description and first experience. J Urol, 2003. 170:1905-7.

24 Fall M, Carlsson CA, Erlandson BE. Electrical stimulation in interstitial cystitis. J Urol, 1980. 123:192-5.

25 Caputo RM, Benson JT, McClellan E. Intravaginal maximal electrical stimulation in the treatment of urinary incontinence. J Reprod Med, 1993. 38:667-71.

26 Spruijt J, Vierhout M, Verstraeten R, Janssens J, Burger C. Vaginal electrical stimulation of the pelvic floor: a randomized feasibility study in urinary incontinent elderly women. Acta Obstet Gynecol Scand, 2003. 82:1043-8.

27 Pal F, Heesakkers JP, Bemelmans LH. Current opinion on the working mechanisms of neuromodulation in the treatment of lower urinary tract dysfunction. Current Opinion in Urology, 2006. 2006:261-267.

28 Knowles $\mathrm{CH}$, Horrocks EJ, Bremner SA, et al. Percutaneous tibial nerve stimulation versus sham electrical stimulation for the treatment of faecal incontinence in adults (CONFIDeNT): a double-blind, multicentre, pragmatic, parallel-group, randomised controlled trial. Lancet, 2015. 386:1640-8.

29 Biemans JM, van Balken MR. Efficacy and effectiveness of percutaneous tibial nerve stimulation in the treatment of pelvic organ disorders: a systematic review. Neuromodulation, 2013. 16:25-33; discussion 33 .

30 Gupta P, Ehlert M, Sirls L, Peters K. Percutaneous Tibial Nerve Stimulation and Sacral Neuromodulation: an Update. Curr Urol Rep 2015;16:1-6.

31 Schmidt RA, Kogan BA, Tanagho EA. Neuroprostheses in the management of incontinence in myelomeningocele patients. J Urol, 1990. 143:779-82.

32 Spinelli M, Malaguti S, Giardiello G, Lazzeri M, Tarantola J, Van Den Hombergh U. A new minimally invasive procedure for pudendal nerve stimulation to treat neurogenic bladder: description of the method and preliminary data. Neurourol Urodyn, 2005. 24:305-9.

33 Peters KM, Killinger KA, Boguslawski BM, Boura JA. Chronic pudendal neuromodulation: expanding available treatment options for refractory urologic symptoms. Neurourol Urodyn, 2010. 29:1267-71.

34 van Kerrebroeck PE, van Voskuilen AC, Heesakkers JP, et al. Results of sacral neuromodulation therapy for urinary voiding dysfunction: outcomes of a prospective, worldwide clinical study. J Urol, 2007. 178: 2029-34. 
35 Siddiqui NY, Wu JM, Amundsen CL. Efficacy and adverse events of sacral nerve stimulation for overactive bladder: A systematic review. Neurourol Urodyn, 2010. 29 Suppl 1: p. S18-23.

36 Siegel S, Noblett K, Mangel J, et al. Results of a prospective, randomized, multicenter study evaluating sacral neuromodulation with InterStim therapy compared to standard medical therapy at 6-months in subjects with mild symptoms of overactive bladder. Neurourol Urodyn, 2015. 34:224-30.

37 Guo ZF, Liu Y, Hu GH, Liu H, Xu YF. Transcutaneous electrical nerve stimulation in the treatment of patients with poststroke urinary incontinence. Clin Interv Aging, 2014. 9:851-6.

38 Gaziev G, Topazio L, Iacovelli V, et al. Percutaneous tibial nerve stimulation (PTNS) efficacy in the treatment of lower urinary tract dysfunctions: a systematic review. BMC Urol, 2013. 13(61).

39 Datta SN, Chaliha C, Singh A, et al. Sacral neurostimulation for urinary retention: 10-year experience from one UK centre. BJU Int, 2008. 101: 192-6.

40 Vandoninck V, van Balken MR, Finazzi Agr E, et al. Posterior tibial nerve stimulation in the treatment of idiopathic nonobstructive voiding dysfunction. Urology, 2003. 61:567-572.

41 Bharucha AE, Dunivan G, Goode PS, et al. Epidemiology, Pathophysiology, and Classification of Fecal Incontinence: State of the Science Summary for the National Institute of Diabetes and Digestive and Kidney Diseases (NIDDK) Workshop. Am J Gastroenterol, 2015. 110:127-136.

42 Bezerra LR, Vasconcelos Neto JA, Vasconcelos CT, et al. Prevalence of unreported bowel symptoms in women with pelvic floor dysfunction and the impact on their quality of life. Int Urogynecol J, 2014. 25:927-33.

43 Thin NN, Horrocks EJ, Hotouras A, et al. Systematic review of the clinical effectiveness of neuromodulation in the treatment of faecal incontinence. The Br J Surg, 2013. 100:1430-47.

44 Thomas GP, Dudding TC, Rahbour G, Nicholls RJ, Vaizey CJ. Sacral nerve stimulation for constipation. Br J Surg, 2013. 100:174-81.

45 Thomas GP, George AT, Dudding TC, Nicholls RJ, Vaizey CJ. A pilot study of chronic pudendal nerve stimulation for faecal incontinence for those who have failed sacral nerve stimulation. Tech Coloproctol, 2014. 18:731-7.

46 Edenfield AL, Amundsen CL, Wu JM, Levin PJ, Siddiqui NY. Posterior tibial nerve stimulation for the treatment of fecal incontinence: a systematic evidence review. Obstet Gynecol Surv, 2015. 70:329-41.

47 Hanno P, Andersson KE, Birder L, Elneil S, Kanai A, Pontari M. Chronic pelvic pain syndrome/bladder pain syndrome: Taking stock, looking ahead: ICI-RS 2011. Neurourol Urodyn, 2012. 31:375-83.

48 Marcelissen T, Jacobs R, van Kerrebroeck PE, de Wachter S. Sacral neuromodulation as a treatment for chronic pelvic pain. J Urol, 2011. 186:387-93.

49 Kabay S, Kabay SC, Yucel M, Ozden H. Efficiency of posterior tibial nerve stimulation in category IIIB chronic prostatitis/chronic pelvic pain: a Sham-Controlled Comparative Study. Urol Int, 2009. 83:33-8. 
CHAPTER 2

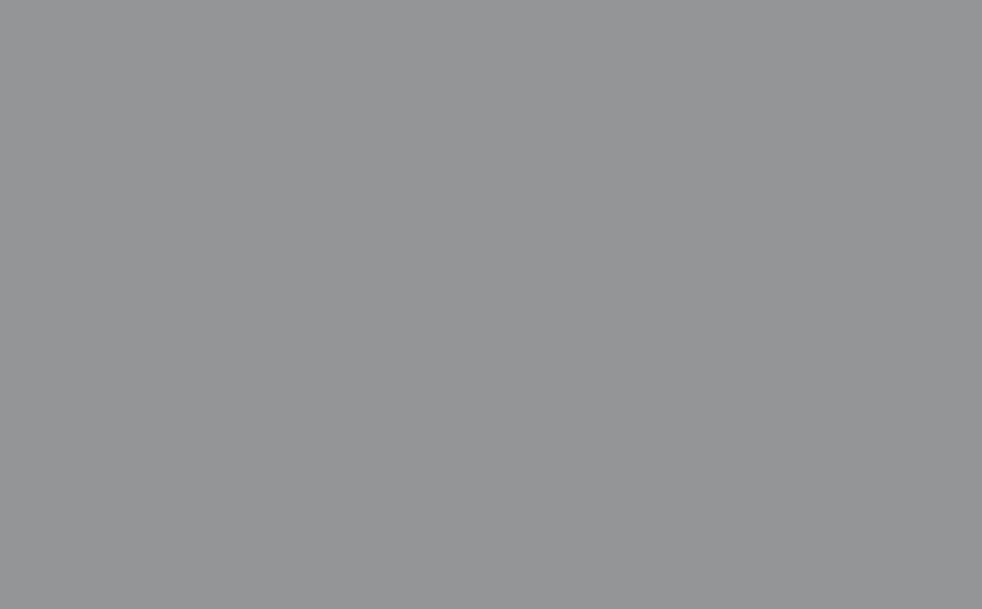




\section{Sacral Nerve Stimulation for Neuromodulation of the Lower Urinary Tract}

Dennis J.A.J. Oerlemans, Philip E.V.A. Van Kerrebroeck Neurourology and Urodynamics 27:28-33 (2008) 


\section{$2 \cdot 1$}

\section{ABSTRACT}

Patients with symptoms of overactive bladder syndrome or non-obstructive urinary retention, refractory to conservative therapy, can nowadays be treated minimally invasively with sacral nerve stimulation (SNS). The use of electric currents to treat urological pathology has a long history but SNS therapy only received FDA approval in 1997. The mechanisms of action are still not known so there are different theories explaining the modulation effect. Recent studies have shown a central modulation effect. Predictive factors which can help to identify the perfect candidates are not known. Over the years the technique of SNS has become less invasive and because of two stage implantation test results have proven to be more reliable. The clinical results for this therapy have proven to be safe and effective and with the technical improvements over the years the re-operation and complication rates have decreased significantly. The clinical results have led to expanding indications because of positive effects in other symptoms. In the field of urology this has resulted in the use of SNS therapy for interstitial cystitis, neurogenic lower urinary dysfunction, and pediatric voiding dysfunction. In the field of gastro-intestinal pathology, SNS therapy is used to treat faecal incontinence and constipation. 


\section{$2 \cdot 2$}

\section{INTRODUCTION}

Chronic types of lower urinary tract dysfunction, including urgency incontinence (UI) and urgency-frequency (UF) and non-obstructive urinary retention (UR), still present a therapeutic challenge. Most patients are initially treated with conservative therapies including bladder retraining, pelvic floor exercises and biofeedback. In the majority of patients this standard regimen is supported with pharmacological therapy (anticholinergics). However, approximately 40\% either do not achieve an acceptable level of therapeutic benefit or remain completely refractory to treatment. Alternative surgical procedures such as bladder transsection, transvesical phenol injection of the pelvic plexus, augmentation cystoplasty and even urinary diversion have been advocated for these chronic conditions. However these procedures have variable efficacy and have been associated with significant morbidity and risk. Therefore research into the use of electrical currents for the treatment of lower urinary tract dysfunction has been initiated.

In 1878, Saxtorph reported intravesical electrostimulation in patients with an acontractile bladder and complete urinary retention. ${ }^{1}$ He inserted a special catheter with a metal electrode transurethrally. Later both Ascoli ${ }^{2}$ and Katona ${ }^{3}$ applied electrostimulation in patients with chronic neurogenic retention and neurogenic overactivity. In the field of urology electrical currents were and are used particularly in the bladder, the pelvic floor muscles and the sacral roots. ${ }^{4-6}$ Electrostimulation of the sacral nerves was developed to empty the bladder and later post stimulus voiding was developed.-10 Brindley developed an electrode for long-term stimulation of the spinal roots and the first patient was implanted in $1972 .{ }^{11}$ The first paraplegic patients were implanted in 1978, using direct stimulation. ${ }^{12}$ A study by Nashold et al. reported on a successful implantation of a neural prosthesis in the sacral segment of the spinal cord..$^{13}$ The implant was used to activate voiding in a patient with spinal cord injury. Jonas and Tanagholo tried to improve this prosthesis because this type of stimulation resulted not only in bladder contractions but also in urinary sphincter contractions as 
well. ${ }^{9}$ Later Tanagho and Schmidt demonstrated that the stimulation of sacral root S3 generally modulates detrusor and sphincter action and could be used in clinical practice. ${ }^{17}$

After two decades of experimentation with sacral root stimulation, finally in October of 1997, sacral neuromodulation for treatment of refractory UI was approved by the Food and Drug Administration in the United States. More than 25,00o patients have undergone SNS since then.

The stimulation of afferent nerve fibers by electrical currents modulates reflex pathways involved in the filling and evacuation phase of the micturition cycle through spinal circuits mediating somato-visceral interactions within the sacral spinal cord. SNS is proposed to activate or 'reset' the somatic afferent inputs that play a pivotal role in the modulation of sensory processing and micturition reflex pathways in the spinal cord..$^{18,19}$ As beneficial effects can be demonstrated at intensities of stimulation that do not activate movements of striated muscle, the afferent system is the most likely affected..$^{20}$ Fowler et al. demonstrated that the anal sphincter reaction seen by acute testing is the result of an afferent mediated response. ${ }^{21}$

UR and dysfunctional voiding can be resolved by inhibition of the guarding reflexes. Detrusor overactivity can be suppressed by one or more pathways, that is, direct inhibition of bladder pre-ganglionic neurons, as well as inhibition of interneuronal transmission in the afferent limb of the micturition reflex. ${ }^{19}$

Recent research with PET-studies indicates that at the level of the brain, the activity of centers in the paraventricular gray involved in activation or inhibition of the micturition reflex, can be enhanced or reduced by SNS. This results in up- or down-grading of lower urinary tract activity. ${ }^{22-24}$ Blok et al. reported on the acute and chronic effects of SNS on the brain in patients with urge-incontinence. They registered differences between newly and chronically implanted patients in brain areas involved in sensory and motor learning. No differences were seen in regional cerebral blood flow $(\mathrm{rCBF})$ in areas that are part of the micturition reflex. Changes in $\mathrm{rCBF}$ were seen in specific areas: areas known to be involved in micturition and areas involved in awareness and consciousness. Acute SNS modulates sensorimotor learning areas and these become less active during chronic SNS. ${ }^{25}$ 


\section{2-3 \\ SELECTING PATIENTS FOR SNS}

All patients with symptoms of voiding dysfunction and who cannot be helped by other measures should be considered for SNS. Patient selection begins with a careful history, physical examination, routine urine tests, and very importantly, voiding diaries. Voiding diaries are a valuable instrument for selection and have to be filled out carefully. Urodynamics are used to identify the patients with detrusor overactivity with or without urinary leakage or UR. Koldewijn et al.studied predictors of success in 100 test stimulation patients but could not identify any. ${ }^{26}$ Scheepens et al. studied data from 211 patients who underwent a test stimulation (percutaneous nerve evaluation [PNE]) to determine the clinical parameters that can enhance the prediction of PNE success. ${ }^{27}$ They found that intervertebral disk prolapse (IPD) surgery, duration of complaints, neurogenic bladder dysfunction, and UI were significant predictive factors. IPD surgery can compromise the sacral root function and can therefore cause UI and UR. ${ }^{28,29}$ In the study by Scheepens et al. patients who underwent IPD surgery had a 3.7 times higher chance of a positive test. Patients with UI responded 2.51 times better to the PNE test. Duration of complaints for more than 7 months and neurogenic bladder dysfunction were both found to be negative predictors of success. However, a PNE remains necessary to evaluate a patient's chance of permanent implant success objectively.

Cohen et al. recently published a study on motor versus sensory response. They concluded that a good motor response during implantation was a predictive factor (in 95\% of successfully treated patients) for success while a sensory response was not. All these patients were implanted under local anesthesia but with intravenous sedation therefore the sensory perception of these patients may be unreliable. ${ }^{30}$

Although not clearly reported before, it is known that a substantial portion of the patients selected for SNS therapy have a history of psychological dysfunction and/or sexual abuse. Weil et al. reported that special attention is needed for this group of patients..$^{31}$ They noted that patients with a history of psychological 
disorders, who had a good response during temporary test-stimulation, had a far greater chance of losing the primary effect after permanent implantation. Of these patients $82 \%$ showed a poor result after definitive implantation compared to $28 \%$ of the patients without a history of psychological disorders. Besides this lack of effect $25 \%$ of the re-operations were done in this group, most of them with no effect. Psychological testing or psychiatric evaluation in case of doubt was advised before implantation of a permanent system.

A study by Everaert et al.showed similar findings. ${ }^{32}$ In this study the twostage procedure was compared with the single-stage procedure. In the twostage implant group there were no failures during the first stage while in the single-stage procedure three patients had an immediate failure. They suggested that these results might be strongly influenced by psychological factors. Mental disorders were not related to objective or subjective success but these cofactors surely interfere with symptomatology and therefore co-influence the results of therapy. 


\section{4 \\ TECHNIQUE OF SACRAL NEUROMODULATION}

SNS incorporates a temporary test stimulation procedure that allows patients and physicians to assess SNS over a trial period. ${ }^{33}$ This test stimulation, also known as a PNE test, is conducted as an outpatient procedure. It is preferably performed under local anesthetic and comprises two steps, acute testing and the home evaluation phase. The original technique was described by Schmidt et al. ${ }^{33}$ A test needle is inserted into the third sacral foramen to stimulate the sacral root. During 4-14 days patients are tested with a temporary lead. Lead migration is a known complication of this test, other complications are technical failures or pain. ${ }^{34}$

Some patients who fail a PNE test are still good candidates for SNS therapy. For this reason a two-stage implant technique was developed. ${ }^{35} \mathrm{With}$ this technique a permanent electrode is implanted and connected to an external stimulator. Less lead migration and a longer test period made it possible to separate non-responders from technical failures. Using this two stage implant technique 8 out of 10 patients who failed a PNE test had a good result with SNS therapy and were implanted with a permanent system. Also long-term results for patients implanted with this technique are promising. ${ }^{36} \mathrm{~A}$ less invasive technique in combination with a newly designed self-anchoring lead made it possible to test patients with this two-stage technique. ${ }^{37,38}$ This 'tined lead' has four sets of self anchoring tines and made a minimally invasive percutaneous placement possible. The procedure can be performed under local anesthesia, requiring no additional incision and no additional anchoring. Besides these advantages this method of implantation made it possible to test the sensitive responses during implantation resulting in a reduction of operating time. The 'tined lead' staged implant technique is widely used in Europe and the US. ${ }^{39}$

The acute phase, whether a PNE or Tined Lead procedure is performed, is used to test the neural integrity, therefore sensory and motor responses should be obtained during this test. The motor responses are important to identify the correct sacral root. Typical S3 stimulation results in bellows movement of 
the pelvic floor, plantar flexion of the great toe and paraesthesia in the rectum, perineum, scrotum, or vagina. Stimulation of the other sacral roots results in different motor responses: S2 stimulation results in clamp movement or twisting and pinching of the anal sphincter and plantar flexion with lateral rotation of the entire foot; $\mathrm{S}_{4}$ stimulation results in bellows motion of the pelvic floor, no lower extremity activity and a sensation of pulling in the rectum. ${ }^{40}$

It can be difficult to palpate the bony landmarks necessary to identify the right puncture place in obese patients. Fluoroscopy can then be used for S3 localization. More importantly, the use of lateral imaging helps to determine the depth required for implanting the $S_{3}$ lead. The use of fluoroscopic localization of $\mathrm{S}_{3}$ allowed the introduction of a tined $\mathrm{S}_{3}$ lead and transformed the placement of a lead from an open procedure to a completely percutaneous one. ${ }^{37,38} \mathrm{The}$ widely adopted percutaneous use of a tined lead approach abandoned the need for fixation of the lead by methods such as bone anchors.

If the patient's symptoms improve by at least $50 \%$ then the patient is a candidate to undergo the stage II or permanent step in which the permanent implantable pulse generator (IPG) is implanted in the soft tissue of the patient's buttock.

Relatively low amplitudes (o $-3.0 \mathrm{~V}$ ) are sufficient for stimulation of the somatic nerve fibers and to minimize the potential for nerve damage due to over-stimulation. Within the recommended stimulation parameters $(210 \mathrm{msec}$, $10-16 \mathrm{~Hz}$ ) continuous stimulation is possible without pain sensation. 


\section{5 \\ UNILATERAL OR BILATERAL STIMULATION}

Although temporary and chronic SNS can result in significant permanent clinical improvement, some patients improve only partially or temporarily. ${ }^{41,42}$ For these patients several methods have been developed to improve the results. ${ }^{37-39}$ The most widely accepted method to test a patient for SNS therapy is unilateral stimulation. In some clinics bilateral stimulation has been suggested as a method to obtain better results. ${ }^{43,44}$ The bilateral innervation of the bladder is the basis for this type of intervention. ${ }^{45,46}$ Animal studies were performed to find a scientific basis for the application of bilateral stimulation. Schultz-Lampel et al. suggest that bilateral SNS can be a more effective technique for voiding dysfunction. ${ }^{47}$ They conclude that bilateral stimulation may be more effective at lower stimulation intensities with positive side-effects such as longer stimulator-battery life and less potential nerve damage.

The only prospective randomized crossover trial to compare the unilateral approach with bilateral sacral nerve stimulation was performed by Scheepens et $a l .4^{8}$ In this study 33 patients with chronic voiding dysfunction underwent unilateral as well as bilateral test stimulation to assess the possible advantages of bilateral stimulation. All patients were stimulated during at least $72 \mathrm{hr}$ in a unilateral and a bilateral setting with a washout period of at least $48 \mathrm{hr}$ between these two test periods. Standardized voiding records were used and urine was measured using standard measuring cups. They analyzed results of 12 patients with UI and 13 patients with non-obstructive UR. They did not find any significant differences comparing the results of unilateral with bilateral stimulation. However, two patients from the retention group started voiding during bilateral stimulation, while during unilateral stimulation they were still in complete retention. The reason for this result could be that with bilateral stimulation sufficient sacral nerve afferents are stimulated to achieve a marked effect at the central level.

In conclusion unilateral stimulation should be performed before bilateral sacral stimulation is considered. However, a bilateral test stimulation could 
be indicated when a unilateral test fails. ${ }^{48,49}$ Further research with clinical follow-up could identify suitable patients for bilateral sacral nerve stimulation. 


\section{6 \\ CLINICAL RESULTS}

In 1999 a prospective randomized study was published in which the results of SNS therapy for UI were evaluated..$^{\circ}$ In total 76 patients were treated in a multi-center trial; 34 patients were implanted and received chronic stimulation for 6 months, after which they completed a therapy evaluation test (on vs. off), 42 patients in a delay group were treated with standard medical therapy for 6 months and were offered implantation after this period. After 6 months the number of daily incontinence episodes, the number of daily replaced diapers and the severity of incontinence was significantly reduced in the stimulation group. In the stimulation group 16 patients (47\%) were completely dry and 10 patients (29\%) showed a greater than $50 \%$ reduction in incontinence episodes. In the delay group (controls) the average incontinence episodes increased during 6 months of conservative therapy from 2.63 .5 heavy episodes a day at baseline to 3.9 3.8 heavy episodes a day at 6 months. After 18 months the efficacy appeared to be sustained. During the therapy evaluation at 6 months the stimulation group returned to baseline symptoms when stimulation was stopped..$^{51}$

Hassouna et al. reported in 2000 on the treatment of UF symptoms with SNS therapy. In total 51 patients, a stimulation group of 25 patients and a control group of 26 patients, enrolled in this multicenter trial. All these patients had been tested with a PNE test and showed satisfactory responses. The stimulation group was implanted directly after this test and the control group was implanted after a 6 month delay period. Statistically significant improvements were seen in the stimulation group for diary parameters such as: number of voids daily $(16.9 \pm 9.7$ to $9.3 \pm 5.1)$, volume per void $(118 \pm 74$ to $226 \pm 124 \mathrm{ml})$ and degree of urgency (rank $2.2 \pm 0.6$ to $1.6 \pm 0.9$ ). In the control group no significant changes were seen. After 6 months the stimulation group underwent an evaluation test and urinary symptoms returned to baseline when stimulation was turned off. After reactivation of the stimulation sustained efficacy was seen at 12 and 24 months.

A report of the use of SNS in UR was published in 2001 by Jonas et al..$^{2} 177$ 
patients with UR refractory to conservative therapy were enrolled in this multicenter trial between 1993 and 1998. Thirty-seven patients were assigned to treatment and 31 to the control group. At 6 months the stimulation group showed $69 \%$ elimination of catheterization and an additional $14 \%$ with greater than $50 \%$ reduction in catheter volume per catheterization. Temporary inactivation (3 days) of SNS therapy resulted in significant increase in residual volume. The effectiveness of SNS therapy was sustained for 18 months after implantation.

The first medium term follow-up results of the above mentioned patient-series were published in 2000. ${ }^{34}$ Results were reported for $1.5-3$ years of follow-up. Of 41 UI patients $59 \%$ showed a greater than $50 \%$ reduction in leaking episodes with $46 \%$ of these patients being completely dry after 3 years. After 2 years of follow-up $56 \%$ of the UF patients showed a greater than $50 \%$ reduction in voids per day. In the retention group $70 \%$ of 42 patients showed a greater than $50 \%$ reduction in catheter volume per catheterization.

During the MDT-103 trial depression and health related quality of life (HRQOL) was assessed in 89 patients. ${ }^{53}$ Patients were divided to a direct implant group or a delayed implant group. At baseline they noted detectable levels of depression in $73 \%$ of all patients. After 3 months patients in the implanted group had a significant improvement in depression scores. These improved scores remained at the 6-and 12-month visits. The scores on the SF-36 questionnaire, a questionnaire to investigate pain, vitality, physical functioning, social functioning, and mental health, increased in the implant group for role-physical, pain, and social functioning. This study demonstrated the serious impact unresolved voiding dysfunction has on quality of life. SNS was associated with significant improvement in depression and HRQOL.

Recently, the 5-year follow-up results of patients included in the trial in order to obtain FDA approval were analyzed. Of 163 patients enrolled, 152 have been implanted. Of the 163 patients, 103 (64\%) had UI, 28 (17\%) UF, and 31 (19\%) UR. Voiding diaries were collected annually over 5 years. For UI patients, the mean number of leaking episodes per day declined from $9.6 \pm 6$.0 to $3.9 \pm 4$.o. For UF patients, mean voids per day decreased from $19.3 \pm 7.0$ to $14.8 \pm 7$.6. Mean volume voided per void increased from $92.3 \pm 52.8$ to $165.2 \pm 147.7 \mathrm{ml}$. In the UR group the average number of catheterizations per day decreased from $5.3 \pm 2.8$ at baseline to $1.9 \pm 2.8$ at 5 years post implant. No life-threatening or irreversible adverse events occurred. Of 152 patients, 102 experienced 31 device-related and 240 therapy-related adverse events. Among these therapy related events, the most frequently reported event was new pain or undesirable change in stimulation (6o times in 41 patients). Pain at the implant site or related to the implanted pulse 
generator (IPG) was the second most reported event (40 times in 30 patients). However an important finding in this study is the high correlation between the 1 - and 5 -year success rates. Of the implanted patients, $84 \%$ with UI, $71 \%$ with UF and $78 \%$ with UR continued to have a successful outcome at 5 -year follow-up if successful at 1 year. ${ }^{54}$

Different groups have published their long term results in recent years. ${ }^{55-57}$ They all conclude that SNS therapy is safe and effective (Table 1).

TABLE 1

Results

\begin{tabular}{lcccc}
\hline Study & \# Patients & Indication & $\begin{array}{c}>\mathbf{5 0 \%} \\
\text { improvement }\end{array}$ & Follow-up \\
\hline Schmidt et al. ${ }^{50}$ & 34 & UI & $76 \%$ & 6 months \\
\hline Hassouna et al..$^{51}$ & 21 & UF & $43 \%$ & 2 years \\
\hline Jonas et al. ${ }^{52}$ & 29 & UR & $83 \%$ & 6 months \\
\hline Siegel et al. ${ }^{34}$ & 41 & UI & $59 \%$ & 3 years \\
\hline & 29 & UF & $56 \%$ & 2 years \\
\hline Dasgupta et al. ${ }^{55}$ & 42 & UR & $70 \%$ & 18 months \\
\hline Voskuilen et al..$^{57}$ & 26 & UR & $77 \%$ & 37 months \\
\hline & 149 & UI/UF/UR & $59.7 \%$ & 46.2 months \\
\hline
\end{tabular}

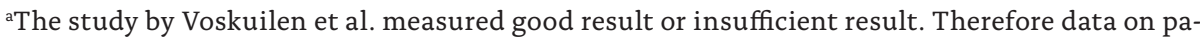
tients with $>50 \%$ improvement are not available. 


\section{7}

\section{COMPLICATIONS}

Most of the studies mentioned in the clinical results section, reported on complications during SNS. Siegel et al. ${ }^{34}$ summarized the complications in patients that were included in the original trials of SNS. The complications were by PNE related and implant related. Of the 914 test stimulation procedures done on the 581 patients, 181 adverse events occurred in 166 of these procedures $(18.2 \%$ of the 914 procedures). The vast majority of complications were related to lead migration (108 events, $11.8 \%$ of procedures). Technical problems and pain represented $2.6 \%$ and $2.1 \%$ of the adverse events. For the 219 patients who underwent implantation of the permanent system, the following adverse events were seen during follow-up: pain at neurostimulator site (15.3\%), new pain (9\%), suspected lead migration (8.4\%), transient electric shock (5.5\%), pain at lead site (5.4\%), adverse change in bowel function (3.0\%) and some less frequent events like technical problems, device problems, change in menstrual cycle, and others. Surgical revisions of the implanted neurostimulator or lead system were performed in $33.3 \%$ of cases (73 of 219 patients) to resolve an adverse event. Mostly this was done to relocate the stimulator because of pain or because of suspected lead migration. No serious adverse events, side effects or permanent injury were reported.

Recently our long term follow-up results with complication rates were published.57 Of 149 patients analyzed, 107 had overactive bladder symptoms and 42 had urinary retention. Mean follow-up was 64.2 ( $\mathrm{SD}=38.5)$ months. In the whole group 194 adverse events occurred. Six patients had infection in their implanted system and one was explanted for infection. Most events could be solved by giving advice or by reprogramming the stimulator. One hundred and twenty-nine reoperations were performed and 21 patients had their system explanted. The most frequent surgical procedures performed were repositioning of the IPG, revision of the electrode because of suspected lead migration, and reoperation for parameter changes in patients implanted with the Itrell-I IPG (the first model used for SNS). Analysis of the data shows a striking difference in the incidence of reoperations, but small differences in subjective results in 
the groups of patients implanted before or after 1996, suggesting that a proactive approach towards adverse events is worthwhile. In our experience with the tined lead implantation we see a clear decrease in reoperation rate..$^{8}$ Of 39 patients implanted with the tined lead Voskuilen van et al. described, there were seven severe adverse events in the medium term and three of these needed a re operation. Three patients could be treated with one or two reprogramming sessions. Three patients had a reoperation to reposition the IPG after complaints of pain. These three patients had good results afterwards. One patient with an incomplete spinal cord lesion had no benefit of the implanted system.

Of 161 patients implanted with the tined lead between July 2002 and September 2004. Hijaz et al.described the complications seen in their institute. ${ }^{59}$ They used three categories for complications: infections, mechanical problems, and response related dysfunction. In total they reported 17 explantations (10.5\%). Eight explantations were done due to infection and seven due to loss of effect. In $26(16.1 \%)$ patients they performed a revision after these patients presented with a decrease in clinical response. The reasons for revision were: mechanical (lead) problems, IPG site discomfort, lead migration and infectious causes. These complication rates show a decrease over the years mainly due to technical and procedural improvements. Gaynor-Krupnick et al. ${ }^{60}$ as well as Hijaz and Vasavada ${ }^{61}$ presented an algorithm for evaluation and management of a malfunctioning neuromodulation system. 


\section{8}

\section{EXPANDING INDICATIONS}

With the widespread use, incidental improvements were published for other pathological conditions. Use of SNS for other off-label applications have been reported for treatment of interstitial cystitis, chronic pelvic pain, pediatric voiding dysfunction, and neurogenic lower urinary dysfunction seen in multiple sclerosis.

Long-term results for the use of SNS in neurogenic UI patients were presented by Chartier-Kastler et al. ${ }^{62}$ The results for nine patients were shown with a mean follow-up of 43.6 months. All patients had significant clinical improvement and 5 (56\%) patients were completely dry. The number of leakages per day went from an initial 7.3 to 0.3 (at last follow-up). Frequency improved after implantation from 16.1 to 8 voids per day and the mean volume per void increased from 115 to $249 \mathrm{ml}$.

In 2000 the first papers were published with positive results for the use of SNS in interstitial cystitis. ${ }^{63,64}$ Comiter evaluated the effect of SNS therapy for interstitial cystitis in a prospective study in 2003. ${ }^{65}$ Seventeen out of 25 patients were implanted with a permanent system. After a mean follow-up of 14 months there were significant improvements in daytime frequency, and nocturia improved from 17.1 to 8.7 and 4.5 to 1.1 , respectively $(\mathrm{P}<0.01)$. Mean voided volume increased from 111 to $264 \mathrm{ml}(\mathrm{P}<0.01)$. Average pain score decreased from 5.8 to 1.6 points on a scale of $\mathrm{O}-10(\mathrm{P}<0.01)$. Interstitial Cystitis Symptom and Problem Index scores decreased from 16.5 to 6.8 and 14.5 to 5.4 , respectively $(P<0.01)$.

In 2001, Siegel et al. ${ }^{66}$ implanted 10 patients with chronic pelvic pain. These patients all had a history of at least 6 months of pelvic pain refractory to conventional treatment without a primary complaint of voiding dysfunction. After 9 months of follow-up 9 out of 10 patients had a decrease in most severe pain scores, and after a median follow-up of 19 months 6 out of 10 patients reported significant improvement in pain symptomatology.

After the clinical implication of SNS therapy for voiding dysfunction, Matzel together with Schmidt and Tanagho started to investigate SNS therapy in bow- 
el dysfunction. ${ }^{67,68}$ In a prospective non-randomized multicenter study 37 patients underwent a test stimulation with SNS therapy for faecal incontinence. ${ }^{69}$ Thirty-four patients were implanted with a permanent system. The effect on incontinence was assessed by daily bowel habit diaries and a disease specific quality of life questionnaire. The frequency of incontinent episodes per week decreased from 16.4 to 3.1 at 12 months and to 2.0 at 24 months for both urge and passive incontinence. The mean number of incontinence episodes per week, staining and pad use declined significantly too. Quality of life improved significantly in ASCRS scales, although in the SF-36 scales only social functioning improved significantly.

Jarrett et al. did a systematic review of SNS for faecal incontinence and constipation..$^{70}$ They reported total continence in $41-75 \%$ of the patients, $75-100 \%$ experienced improvement in the incontinence symptoms. The results for patients treated with SNS for constipation discussed in this review seem promising but only limited data is available at this time.

The results of SNS therapy in children with neurogenic bladder dysfunction is described by Guys et al.71 In total 2 children with neurogenic bladder dysfunction, mainly due to spina bifida, enrolled in this prospective randomized controlled trial. Twenty-one patients were treated conservatively while the other 21 patients were treated with SNS therapy. After 12 months no significant improvement in results were seen in the group treated with SNS. The authors stated that probably the intervention group was too small or the bladder dysfunction in these patients too severe.

Pauls et al. recently reported a pilot study to determine if sacral neuromodulation has an effect on the patient's subsequent sexual function..$^{22}$ Eleven patients with a permanent system implanted were questioned about their sexual function before and after implantation. With SNS therapy sexual frequency and Female Sexual Function Index (FSFI) increased significantly. No correlation was found between improvement in urinary symptoms and FSFI scores. 


\section{9}

\section{CONCLUSIONS}

After years of experimental therapy, initiated by Tanagho and Schmidt, SNS is nowadays a widely used therapy. Although the mechanisms of action are still not fully understood, the therapy has been proven effective in the long term. Due to the less invasive technique and other technical improvements it is expected that complication rates will further decrease within the coming years.

The expanding use of SNS therapy in fields other than urology will probably result in FDA approval for gastro-intestinal indications.

Further research, possibly with the help of animal models, has to be performed to understand in a more precise way the mechanism of SNS therapy. Other goals in research could be: patient selection (finding ways to identify the best candidates), the effect of SNS in combined (urological/gynaecological/gastro-enterological) pathology and the effect of bilateral versus unilateral stimulation. 


\section{2-10}

\section{ACKNOWLEDGMENTS}

This work was supported by an educational grant from Medtronic Europe, Tolochenaz, Switzerland and by the WAMU foundation, Maastricht, the Netherlands. 


\section{$2 \cdot 11$ REFERENCES}

1 Saxtorph M. Stricture urethrae-Fistula perinee-Retentio urinae. Clinsk Chirurgi, Gyldendalske Fortag Copenhagen, 1878; pp $265-80$.

2 Ascoli R. Treatment of neurogenic bladder. Acta Urol Belg 1965;33:76 - 83.

3 Katona F. Stages of vegatative afferentation in reorganization of bladder control during intravesical electrotherapy. Urol Int 1975;30:192-203.

4 Boyce WH, Lathem E, Hunt LD. Research related to the development of an artificial electrical stimulator for the paralyzed human bladder: A review. Trans Am Assoc Genitourin Surg 1963;55:81-91.

5 Boyce WH, Lathem JE, Hunt LD. Research related to the development of an artificial electrical stimulator for the paralyzed human bladder: A review. J Urol 1964;91:41-51.

6 Dees JE. Contraction of the urinary bladder produced by electric stimulation. Preliminary Report Invest Urol 1965;15:539-47.

7 Brindley GS. An implant to empty the bladder or close the urethra. J Neurol Neurosurg Psychiatry 1977;40:358-69.

8 Eidelberg E, Bors E, Woodbury CM, et al. Effect of electrical stimulation of dorsal and ventral spinal cord roots on the cat's urinary bladder. Urol Int 1974;29:375 - 81.

9 Jonas U, Heine JP, Tanagho EA. Studies on the feasibility of urinary bladder evacuation by direct spinal cord stimulation. I. Parameters of most effective stimulation. Invest Urol 1975;13:142-50.

10 Jonas $\mathrm{U}$, Tanagho EA. Studies on the feasibility of urinary bladder evacuation by direct spinal cord stimulation. II. Poststimulus voiding: A way to overcome outflow resistance. Invest Urol 1975;13:151-3.

11 Liverpool. Brindley GS. History of the sacral anterior root stimulator, $1969-1982$. Neurourol Urodyn 1993;12:481 - 3 .

12 Nashold BS, Jr., Friedman H, Boyarsky S. Electrical activation of micturition by spinal cord stimulation. J Surg Res 1971;11:144 - 7 .

14 Schmidt RA, Bruschini H, Tanagho EA. Sacral root stimulation in controlled micturition. Peripheral somatic neurotomy and stimulated voiding. Invest Urol 1979;17:130 - 4.

15 Tanagho EA, Schmidt RA. Bladder pacemaker: Scientific basis and clinical future. Urology 1982;20:614- 9 .

16 Tanagho EA, Schmidt RA. Electrical stimulation in the clinical management of the neurogenic bladder. J Urol 1988;140:1331 - 9 .

17 Tanagho EA, Schmidt RA, Orvis BR. Neural stimulation for control of voiding dysfunction: A preliminary report in 22 patients with serious neuropathic voiding disorders. J Urol 1989;142:340 - 5 .

18 Fall M, Lindstrom S. Electrical stimulation. A physiologic approach to the treatment of urinary incontinence. Urol Clin North Am 1991;18:393 - 407. 
19 Leng WW, Chancellor MB. How sacral nerve stimulation neuromodulation works. Urol Clin North Am 2005;32:11 - 8.

20 Vodusek DB, Light JK, Libby JM. Detrusor inhibition induced by stimulation of pudendal nerve afferents. Neurourol Urodyn 1986;5:381 - 9.

21 Fowler CJ, Swinn MJ, Goodwin RJ, et al. Studies of the latency of pelvic floor contraction during peripheral nerve evaluation show that the muscle response is reflexly mediated. J Urol 2000;163:881- 3 .

22 Blok BF. Central pathways controlling micturition and urinary continence. Urology 2002;59:13-7.

23 Blok BF, Groen J, Veltman D, et al. Brain plasticity and urge incontinence: PET studies during the first hours of sacral neuromodulation. Neurourol Urodyn 2003;22:490 - 91.

24 Dasgupta R, Critchley HD, Dolan RJ, et al. Changes in brain activity following sacral neuromodulation for urinary retention. J Urol 2005;174:2268 - 72 .

25 Blok BF, Groen J, Bosch JL, et al. Different brain effects during chronic and acute sacral neuromodulation in urge incontinent patients with implanted neurostimulators. BJU Int 2006;98:1238-43.

26 Koldewijn EL, Rosier PF, Meuleman EJ, et al. Predictors of success with neuromodulation in lower urinary tract dysfunction: Results of trial stimulation in 100 patients. J Urol 1994;152:2071- 5 .

27 Scheepens WA, Jongen MM, Nieman FH, et al. Predictive factors for sacral neuromodulation in chronic lower urinary tract dysfunction. Urology 2002; 60:598 - 602.

28 O'Flynn KJ, Murphy R, Thomas DG. Neurogenic bladder dysfunction in lumbar inter-vertebral disc prolapse. Br J Urol 1992;69:38 - 40.

29 Yamanishi T, Yasuda K, Sakakibara R, et al. Detrusor overactivity and penile erection in patients with lower lumbar spine lesions. Eur Urol 1998;34: 360-4.

30 Cohen BL, Tunuguntla HS, Gousse A. Predictors of success for first stage neuromodulation: Motor versus sensory response. J Urol 175:2178 - 80; discussion 2006; 2180 - 71 .

31 Weil EH, Ruiz-Cerda JL, Eerdmans PH, et al. Clinical results of sacral neuromodulation for chronic voiding dysfunction using unilateral sacral foramen electrodes. World J Urol 1998;16:313-21.

32 Everaert K, Kerckhaert W, Caluwaerts $\mathrm{H}$, et al. A prospective randomized trial comparing the 1-stage with the 2-stage implantation of a pulse generator in patients with pelvic floor dysfunction selected for sacral nerve stimulation. Eur Urol 2004;45:649 - 54.

33 Schmidt RA, Senn E, Tanagho EA. Functional evaluation of sacral nerve root integrity. Report of a technique. Urology 1990;35:388 - 92.

34 Siegel SW, Catanzaro F, Dijkema HE, et al. Long-term results of a multicenter study on sacral nerve stimulation for treatment of urinary urge incontinence, urgency-frequency, and retention. Urology 2000;56:87 - 91.

35 Janknegt RA, Weil EH, Eerdmans PH. Improving neuromodulation technique for refractory voiding dysfunctions: Two-stage implant. Urology 1997;49: 358 - 62.

36 Scheepens WA, Van Koeveringe GA, De Bie RA, et al. Long-term efficacy and safety results of the two-stage implantation technique in sacral neuromodulation. BJU Int 2002;90:840 - 5 .

37 Spinelli M, Giardiello G, Arduini A, et al. New percutaneous technique of sacral nerve stimulation has high initial success rate: Preliminary results. Eur Urol 2003;43:70 - 4 .

38 Spinelli M, Giardiello G, Gerber M, et al. New sacral neuromodulation lead for percutaneous implantation using local anesthesia: Description and first experience. J Urol 2003;170:1905 7.

39 Spinelli M, Weil E, Ostardo E, et al. New tined lead electrode in sacral neuromodulation: Experience from a multicentre European study. World J Urol 2005;23:225 - 9. 
40 Daneshgari F. Applications of neuromodulation of the lower urinary tract in female urology. Int Braz J Urol 2006;32:262 - 72.

41 Bosch JL, Groen J. Sacral nerve neuromodulation in the treatment of patients with refractory motor urge incontinence: Long-term results of a prospective longitudinal study. J Urol 2000;163:1219- 22.

42 Weil EH, Ruiz-Cerda JL, Eerdmans PH, et al. Sacral root neuromodulation in the treatment of refractory urinary urge incontinence: A prospective randomized clinical trial. Eur Urol 2000;37:161 - 71 .

43 Braun P, Seif C, Scheepe J, et al. Chronic sacral bilateral neuromodulation. Using a minimal invasive implantation technique in patients with disorders of bladder function. Urologe A 2002;41:44- 7 .

44 Hohenfellner M, Schultz-Lampel D, Dahms S, et al. Bilateral chronic sacral neuromodulation for treatment of lower urinary tract dysfunction. J Urol 1998;160:821 - 4.

45 Diokno AC, Davis R, Lapides J. The effect of pelvic nerve stimulation on detrusor contraction. Invest Urol 1973;11:178 - 81.

46 Ingersoll EH, Jones LL, Hegre ES. Effect on urinary bladder of unilateral stimulation of pelvic nerves in the dog. Am J Physiol 1957;189:167 - 72.

47 Schultz-Lampel D, Jiang C, Lindstrom S, et al. Neurophysiologische Effekte unilateraler und bilateraler sakraler Neuromodulation. Aktuel Urol 1998;29:354 - 60.

48 Scheepens WA, de Bie RA, Weil EH, et al. Unilateral versus bilateral sacral neuromodulation in patients with chronic voiding dysfunction. J Urol 2002; 168:2046 - 50.

49 van Kerrebroeck PE, Scheepens WA, de Bie R, et al. European experience with bilateral sacral neuromodulation in patients with chronic lower urinary tract dysfunction. Urol Clin North Am 2005;32:51 - 7 .

50 Schmidt RA, Jonas U, Oleson KA, et al. Sacral nerve stimulation for treatment of refractory urinary urge incontinence. Sacral Nerve Stimulation Study Group. J Urol 1999;162:352 - 7.

51 Hassouna MM, Siegel SW, Nyeholt AA, et al. Sacral neuromodulation in the treatment of urgency-frequency symptoms: A multicenter study on efficacy and safety. J Urol 2000;163:1849 -54 .

52 Jonas U, Fowler CJ, Chancellor MB, et al. Efficacy of sacral nerve stimulation for urinary retention: Results 18 months after implantation. J Urol 2001; 165:15 - 9.

53 Das AK, Carlson AM, Hull M. Improvement in depression and health-related quality of life after sacral nerve stimulation therapy for treatment of voiding dysfunction. Urology 2004;64:62 - 8 .

54 van Kerrebroeck PE, van Voskuilen AC, Heesakkers JP, et al. Results of sacral neuromodulation therapy for urinary voiding dysfunction: outcomes of a prospective, worldwide clinical study. J Urol 2007;178:2029-34.

55 Dasgupta R, Wiseman OJ, Kitchen N, et al. Long-term results of sacral neuromodulation for women with urinary retention. BJU Int 2004;94:335 - 7. 56. Elhilali MM, Khaled SM, Kashiwabara T, et al. Sacral neuromodulation: Long-term experience of one center. Urology 2005;65:1114- 7 .

57 van Voskuilen AC, Oerlemans DJ, Weil EH, et al. Long term results of neuromodulation by sacral nerve stimulation for lower urinary tract symptoms: A retrospective single center study. Eur Urol 2006;49:366 - 72. 58. Voskuilen van AC, Oerlemans DJ, Weil EH, et al. Medium-term experience of sacral neuromodulation by tined lead implantation. BJU Int 2007;99:107-10.

59 Hijaz A, Vasavada SP, Daneshgari F, et al. Complications and troubleshooting of two-stage sacral neuromodulation therapy: A single-institution experience. Urology 2006;68:533 - 7 . 
6o Gaynor-Krupnick DM, Dwyer NT, Rittenmeyer H, et al. Evaluation and management of malfunctioning sacral neuromodulator. Urology 2006;67: 246-9.

61 Hijaz A, Vasavada S. Complications and troubleshooting of sacral neuromadulation therapy. Urol Clin North Am 2005;32:65 - 9.

62 Chartier-Kastler EJ, Ruud Bosch JL, Perrigot M, et al. Long-term results of sacral nerve stimulation $\left(\mathrm{S}_{3}\right)$ for the treatment of neurogenic refractory urge incontinence related to detrusor hyperreflexia. J Urol 2000;164:1476 - 80.

63 Chai TC, Zhang C, Warren JW, et al. Percutaneous sacral third nerve root neurostimulation improves symptoms and normalizes urinary HB-EGF levels and antiproliferative activity in patients with interstitial cystitis. Urology 2000;55:643-6.

64 Zermann DH, Weirich T, Wunderlich H, et al. Sacral nerve stimulation for pain relief in interstitial cystitis. Urol Int 2000;65:120 - 1 .

65 Comiter CV. Sacral neuromodulation for the symptomatic treatment of refractory interstitial cystitis: A prospective study. J Urol 2003;169: 1369-73.

66 Siegel S, Paszkiewicz E, Kirkpatrick C, et al. Sacral nerve stimulation in patients with chronic intractable pelvic pain. J Urol 2001;166:1742 - 5.

67 Matzel KE, Schmidt RA, Tanagho EA. Neuroanatomy of the striated muscular anal continence mechanism. Implications for the use of neurostimulation. Dis Colon Rectum 1990;33:666 - 73 .

68 Matzel KE, Stadelmaier U, Hohenfellner M, et al. Electrical stimulation of sacral spinal nerves for treatment of faecal incontinence. Lancet 1995;346: 1124 - 7.

69 Matzel KE, Kamm MA, Stosser M, et al. Sacral spinal nerve stimulation for faecal incontinence: Multicentre study. Lancet 2004;363:1270 - 6.

70 Jarrett ME, Mowatt G, Glazener CM, et al. Systematic review of sacral nerve stimulation for faecal incontinence and constipation. Br J Surg 2004;91: 1559-69.

71 Guys JM, Haddad M, Planche D, et al. Sacral neuromodulation for neurogenic bladder dysfunction in children. J Urol 2004;172:1673-6.

72 Pauls RN, Marinkovic SP, Silva WA, et al. Effects of sacral neuromodulation on female sexual function. Int Urogynecol J Pelvic Floor Dysfunct 2007;18: 391-5. 
CHAPTER 3 


\section{Long Term Results of Neuromodulation by Sacral Nerve Stimulation for Lower Urinary Tract Symptoms: A Retrospective Single Center Study}

Anco C. van Voskuilen, Dennis J.A.J. Oerlemans,

Ernest H.J. Weil, Rob A. de Bie, Philip E.V.A. Van Kerrebroeck European Urology 49 (2006) $366-372$ 


\section{3-1}

\section{ABSTRACT}

\section{Objective}

To analyse the influence of technical improvements of Sacral Nerve Stimulation (SNS) on the incidence of surgical interventions and subjective long-term results of SNS.

\section{Methods}

Retrospective, transversal study analyzing records of implanted patients at our department of Urology.

\section{Results}

Of 149 patients analyzed, 107 had overactive bladder symptoms and 42 had urinary retention. Mean follow-up was $64,2(s d=38,5)$ months. In the whole group 194 adverse events occurred. 6 Patients had infection in their implanted system, one was explanted for infection. Most events could be solved by giving advice or by reprogramming the stimulator. 129 reoperations have been performed and 21 patients had their system explanted. Analysis of the data shows a striking difference in the incidence of reoperations, but small differences in subjective results in the groups of patients implanted before or after 1996, suggesting that a proactive approach towards adverse events is worthwhile.

\section{Conclusions}

SNS gives lasting benefit in patients with refractory symptoms of overactive bladder and non-obstructive urinary retention. The differences in outcomes and incidence of reoperation can be attributed to the learning curve and technical and surgical improvements in the application of SNS. 


\section{3-2}

\section{INTRODUCTION}

In 1994 neuromodulation by sacral nerve stimulation (SNS) became available as a new treatment option for patients with refractory urge incontinence, urgency-frequency and nonobstructive urinary retention. ${ }^{1-4}$ The SNS procedure is non-mutilating compared to other surgical procedures. Therefore, it gained a place in the list of therapeutic options a physician can offer to patients with the above-mentioned symptoms. Sacral neuromodulation is placed between non-invasive therapies such as anticholinergics or pelvic floor training and surgical procedures, such as bladder augmentation and urinary deviation. Initially patients were implanted according to the protocol of a multicenter study initiated by the manufacturer of the implanted equipment in order to achieve FDA approval (MDT-103)..$^{-8}$ From early application of SNS until now continuous research is carried out to improve this therapy and to determine the exact mechanism of action. This research has yielded some technical improvements in SNS. In the period covering our study the design of the Implantable Pulse Generator (IPG) has been changed two times, lead design has changed and there have been advancements in surgical techniques. In our experience these advancements have contributed to decreased complications rates as well as increased satisfaction rates in our patients receiving SNS therapy. ${ }^{9-11}$ This study is set up to confirm this hypothesis. 


\section{3-3}

\section{METHODS}

This is a transversal cohort study. The surgery logs of the department of Urology of the University Hospital Maastricht covering the period between 1990 and December 2003 have been scrutinized for patients who received a new definitive neuromodulation system or had revision surgery. Patients were included if they had follow-up of longer than one year and if complete data were available. All patients were implanted according to the open procedure as proposed by Tanagho and Schmidt. ${ }^{12}$ Patients, implanted through the percutaneous tined lead procedure were excluded, as well as patients receiving bilateral stimulation. ${ }^{9}$

All patients had shown successful on screening using the Percutaneous Nerve Evaluation (PNE) with a temporary lead or after a two-stage procedure. ${ }^{13}$ 12 patients had their definitive implant through a 2-stage procedure.

The first series of patients were implanted with the Itrel-I IPG, which was the first model used for SNS. Parameter change was done in an open surgical procedure. The Itrell-II was introduced in 1994 and allowed parameter modification by the physician using an external programmer.

Patients, implanted with the Itrell-II could switch the IPG on and off and choose between two preprogrammed settings with a magnet. Since 1999 the Interstim IPG is used. This latest model is supplied with a patient remote control for switching the device on/off and for changing amplitude within a preprogrammed range. In our opinion this decreases outpatient visits as patients can handle amplitude related events by themselves.

The first model of the implanted lead had separate fixation connectors, which had to be sutured onto the lead. In 1994 a new lead model was introduced with fixation connectors integrated into the lead. From 2000 the IPG was implanted gluteally instead of abdominally, as this placement gave less complications.10

From the files of the implanted patients the following data were collected: type of complaints, date of implantation, date and result of neuromodulation of last check-up, number of adverse events and if adverse events had successfully been addressed, date of reoperations for adverse events. 
Patient complaints were stratified on an 'urge' category and 'retention' category. The urge category comprises patients suffering from urge incontinence or urgency-frequency with or without pelvic pain complaints. The retention category consists of patients with non-obstructive retention, regardless of the cause of the complaints.

The subjective results of the patients at their last visit were categorized as 'good' or as 'insufficient'. The criteria for success were complete and lasting disappearance of symptoms or satisfactory symptoms for the patient.

An insufficient result was noted when the patient was not satisfied, when symptoms recurred, in the case of an uncorrected adverse event or when the system was explanted. If at last follow-up visit the stimulation parameters of the IPG were changed, the chart of the patient was analyzed a second time during the revision period of this manuscript and the result was noted. This is based on the fact that patients are instructed to report back if symptoms do not improve. The patients are encouraged to contact the clinic when they suspect neuromodulation-related complaints.

The number of adverse events the patient had in the period from implant until last follow-up was counted as well. An adverse event was noted when the patient had neuromodulation-related complaints, not solved by changing the stimulation amplitude. Necessary outpatient reprogramming sessions of the stimulator for loss of sensation were only recorded as an adverse event if loss of sensation was accompanied by loss of efficacy. Battery depletion of the IPG was recorded separately if the procedure was not combined with other neuromodulation related surgery.

The data of the patients were analyzed using SPSS 11.5 for Windows (SPSS inc., USA).

Analysis of contributing factors to the results was done using bivariate correlation analysis by Pearson's Chi-square test. Further statistical analysis was performed using the Mann-Whitney test. 


\section{4 \\ RESULTS}

Between October 1990 and December 2002190 patients were implanted. 15 patients were implanted less than a year ago, and therefore not analyzed. The data in the files of 26 patients was incomplete. In 17 of these files data on the implant was missing as these patients were implanted in another hospital and had reoperations in our department. In the remaining 9 files other data on adverse events or reoperations were missing. A total of 149 patients met the inclusion criteria and were analyzed. The group consisted of 27 male $(18,1 \%)$ and 122 female patients $(81,9 \%)$. Among these patients $129(86,6 \%)$ had an idiopathic cause of their symptoms and $20(13,4 \%)$ patients had a neurologic cause of their urinary complaints. Table 1 shows the diagnosis and results of these patients.

Indications for implant were overactive bladder symptoms in 107 (71.8\%) and retention in $42(28.2 \%)$ patients. Mean age at implantation was $46.7(\mathrm{sd}=10.0)$ years, range between 21 and 72 years.

Follow-up ranged between 12 and 154 months after implant, mean follow-up period was $64.2(\mathrm{sd}=38.5)$ months.

The mean follow-up for patients who were implanted for urgency symptoms was 69,8 months $(s d=41.6)$ and for patients with urinary retention 70.5 months $(\mathrm{sd}=38.0)$. In the whole group $89(59.7 \%)$ patients were scored having a good result, 44 (29.5\%) patients had insufficient results and 16 patients (10.7\%) had a reprogramming session at their last visit.

During the revision process of this manuscript the follow-up of these 16 patients after database closure showed that $11(68.8 \%)$ had good results after a period of three to 6 months after their visit and 5 (31.3\%) patients kept insuffcient results after reprogramming. With these 16 patients included a total of 100 patients $(67.1 \%)$ had a good result and $49(32.9 \%)$ had insufficient results. 68 (63.6\%) of the 107 patients with urgency had good results at the time of their last follow-up and $39(36.4 \%)$ patients with urgency had insufficient results. In the retention group of 42 patients $32(76.2 \%)$ patients had good results and 10 patients $(23.8 \%)$ had insufficient results. 
TABLE 1

Diagnosis

\# patients

Result

Result

good

insufficient

\begin{tabular}{llll}
\hline ALS & 1 & 1 & 0 \\
\hline Cauda Equina syndrome & 3 & 1 & 2 \\
\hline Peripheral polyneuropathy & 2 & 2 & 0 \\
\hline Guillain Barre & 1 & 1 & 0 \\
\hline IDP & 5 & 3 & 2 \\
\hline SCI & 1 & 1 & 0 \\
\hline Multiple Sclerosis & 1 & 1 & 0 \\
\hline Paraplegia & 1 & 0 & 1 \\
\hline Spina Bifida & 1 & 1 & 0 \\
\hline Spondyolysis & 1 & 1 & 0 \\
\hline Whiplash & 2 & 1 & 1 \\
\hline Unknown neorologic & 1 & 0 & 1 \\
\hline Total & $20(100 \%)$ & $13(65 \%)$ & $7(35 \%)$ \\
\hline
\end{tabular}

ALS: Amyotrophic Lateral Sclerosis, IDP: Intevertebral Disk Prolaps, SCI: Spinal Cord Injury. 
TABLE 2

Diagnosis

Pain/undesirable change in stimulation 64

Undesirable change in voiding function/Loss of efficacy 42

Oain at IPG implant site 41

\begin{tabular}{ll}
\hline Afverse change in bowel function & 15
\end{tabular}

Suspected lead migration 10

\begin{tabular}{lc}
\hline Suspected device problem (including lead breakage) & 6
\end{tabular}

Infection 6

Technical problem $\quad 5$

Suspected neuropraxia 2

Other 3

$\begin{array}{lr}\text { Total } & 194\end{array}$

The figures described in this table are numbers of adverse events. Some patients had more than one ad-verse event. Pain or undesirable stimulation was only recorded as an adverse event when changing the stimulator amplitude could not solve the problem. Suspected neuropraxia was seen in 2 patients who set their amplitudes very high and was in both patients successfully solved by switching the IPG off for 6 months. The events in the 'Other' category all were with one occurrence. 
In the group of 149 patients with a total of 9562 months of SNS experience 194 adverse events occurred in 106 patients. There were no irreversible or severe adverse events. The most frequently occurring adverse events were changes in stimulation sensation, loss of efficacy and pain at the Implantable Pulse Generator (IPG) site (Table 2).

Of all analyzed patients $48.3 \%$ had at least one reoperation due to an adverse event. A total of 129 reoperations were performed in the total patient group to address adverse events, averaging 0.87 reoperations per patient.

The most frequent surgical procedures were repositioning of the IPG for pain, revision of the electrode because of suspected lead migration and reoperation for parameter change in patients implanted with the Itrell-I IPG. The 129 surgical procedures do not include replacement of the IPG for battery depletion.

19 revisions for battery depletion took place in 18 patients. One patient who was implanted in 1992 has had two revisions for battery depletion. The mean life span of the replaced IPGs in these patients was 73,7 months with a range between 28 to 127 months.

Chi-square correlation analysis between implantation date, the sex of the patient, type of complaints, follow-up duration, number of adverse events and whether adverse events had been resolved at last follow-up and the result at last follow-up was performed.

The analysis showed a significant correlation between result and adverse events being resolved: $0.830(\mathrm{p}=0.000)$. Also the surgery per year ratio and the final results were correlated: $0.397(p=0,000)$.

However an inverse correlation was noted between result and the number of adverse events: $0.355(\mathrm{p}=0.000)$. Patients without adverse events had the highest chance of a good result, but when patients had an adverse event, it was in most cases satisfactorily resolved.

The Chi-square test did not show a significant difference between the results of patients with or without a neurologic cause for their complaints. Analysis of the data shows a clear decrease in the number of the adverse events and reoperations during the study period.

Fig. 1 shows the ratio of revisions and the ratio of adverse events in the patients implanted in each year divided by the number of implanted patients in that year (Table 3 ).

In the patients implanted before 1995 the mean number of reoperations was 1.56, while in the patients implanted after 1995 the mean decreased to 0.49 reoperations. This difference is statistically significant $(\mathrm{p}<0.0001)$.

The difference in the results between both groups changed as well: $60.7 \%$ of 
the patients implanted before 1995 had a good result while $65.9 \%$ of the patients implanted after 1995 had a good result. This difference is not statistically significant (Fig. 2).

In $21(14.1 \%)$ of the 149 patients the neuromodulation system had been removed. The most common indication for explant was loss of efficacy (18 patients). The remaining three patients have been explanted for uncorrectable side effects, one of these patients had an explant due to infection.

Of the explanted patients 15 (71.4\%) had urge symptoms, while 6 patients (28.6\%) suffered from retention. The mean follow-up was 53.6 months ( $\mathrm{sd}=40.7)$, while the follow-up ranged between 12 and 145 months. Statistical analysis in the 21 explanted patients shows a higher mean surgery/year ratio of $0.56(\mathrm{sd}=$ 0.09) than in the non-explanted group with a ratio of $0.12(\mathrm{sd}=0.2)(\mathrm{p}<0.005)$. In 14 patients $(66.7 \%)$ the operation in which the IPG was explanted was their only reoperation, in the remaining explanted patients the number of surgical sessions before the explant ranged between 1 and 11 sessions. 
TABLE 3

The number of implants, adverse events, reoperations and the ratios of revisions/implants and adverse events/ implants for each year

\begin{tabular}{llllll}
\hline Year & \# Implants & \# Revisions & $\begin{array}{c}\text { \# Adverse } \\
\text { events }\end{array}$ & $\begin{array}{c}\text { Revision } \\
\text { ratio }\end{array}$ & $\begin{array}{c}\text { Events } \\
\text { ratio }\end{array}$ \\
\hline 1990 & 4 & 17 & 18 & 4,25 & 4,50 \\
\hline 1991 & 14 & 19 & 24 & 1,36 & 1,71 \\
\hline 1992 & 8 & 10 & 14 & 1,25 & 1,75 \\
\hline 1993 & 9 & 5 & 10 & 0,56 & 1,11 \\
\hline 1994 & 14 & 21 & 26 & 1,50 & 1,86 \\
\hline 1995 & 12 & 10 & 14 & 0,83 & 1,17 \\
\hline 1996 & 12 & 10 & 12 & 0,83 & 1,00 \\
\hline 1997 & 10 & 11 & 16 & 1,10 & 1,60 \\
\hline 1999 & 14 & 9 & 17 & 0,64 & 1,21 \\
\hline 2000 & 16 & 5 & 21 & 0,31 & 1,31 \\
\hline 2001 & 17 & 6 & 17 & 0,35 & 1,00 \\
\hline 14 & 0 & 0 & 0,00 & 0,36 \\
\hline 2003 & 1 & 0,00 & 0,25 \\
\hline
\end{tabular}


FIGURE 1

Revision/implant ratio over time. The ratio of revisions carried out in the patients implanted in each year divided by the number of the implants in each ear. Revisions for empty batteries are excluded.

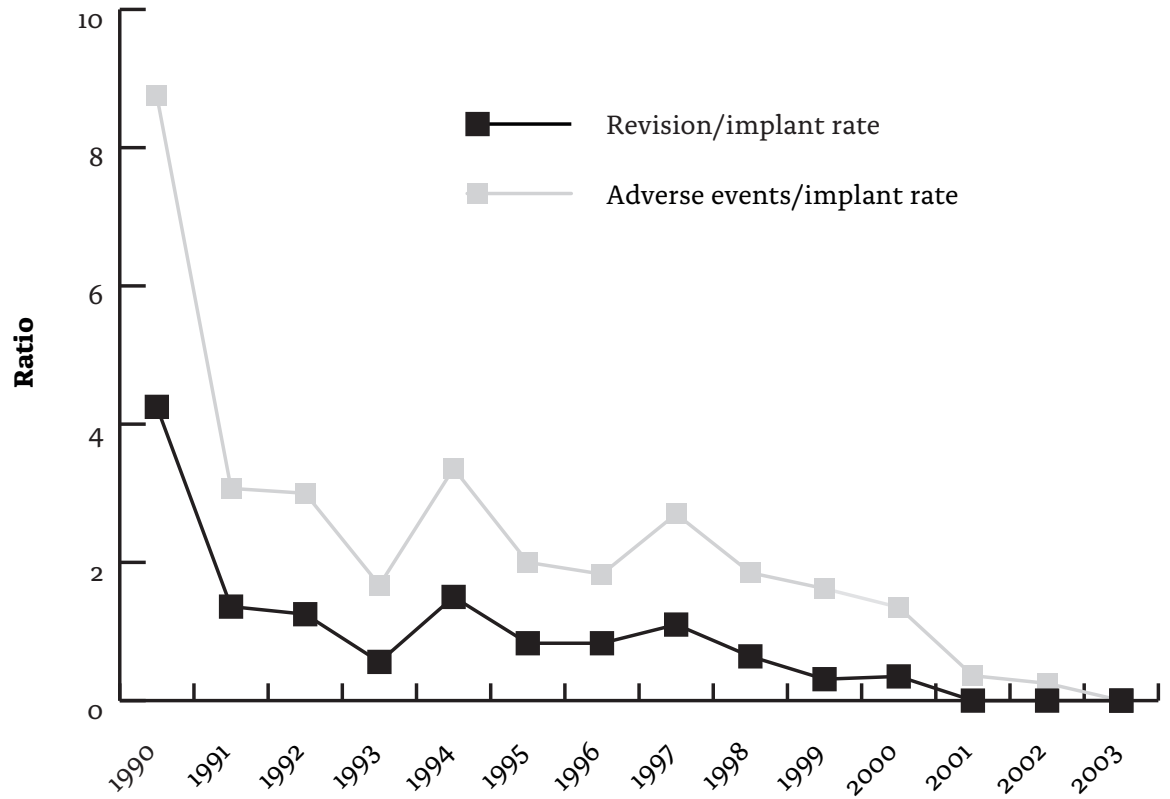

Time 
FIGURE 2

Number of surgical revisions/patient before and after 1995. The amount of patients with surgical revisions of their implanted neuromodulation system before/in 1995 and after 1995.

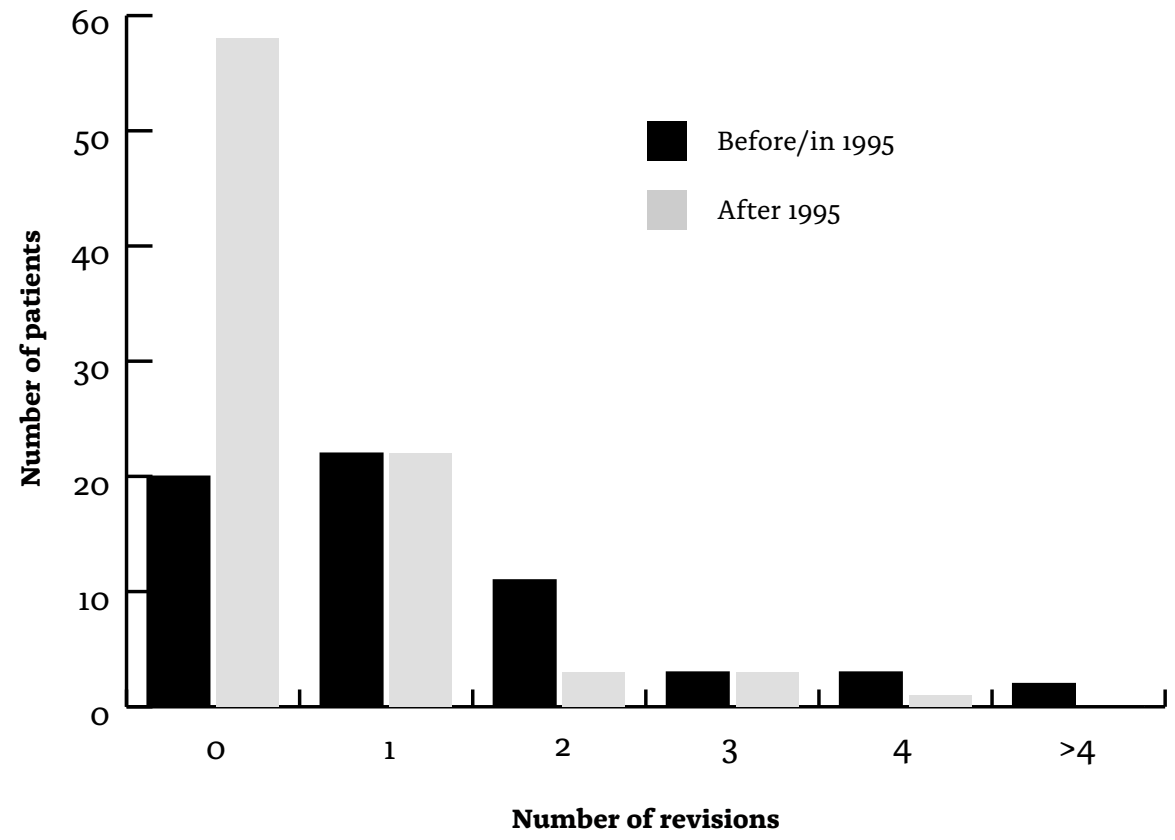




\section{5}

\section{DISCUSSION}

Sacral neuromodulation gives lasting and satisfactory relief in patients with refractory symptoms of overactive bladder and urinary retention. $4,7,14-16$

Because the study setup is retrospective and voiding diaries were not always found in the file, the outcome of the patients was based on patient and physician satisfaction, instead of on voiding diary parameters.

Usually patients are satisfied when they are without symptoms or have very few symptoms. These results, however, are not comparable to other studies because of the subjective measurement of the outcome.

Some retrospective studies have been performed to identify predictive factors regarding the success of the Percutaneous Nerve Evaluation. ${ }^{17,18}$

So far no research has been done about predictive factors for the success of the definitive implant. In this study logistic regression analysis between result and date of implantation, sex of the patient, the type of complaints, follow-up time, the number of adverse events and whether adverse events had been resolved at last follow-up has been carried out. However, none of these factors seemed to predict the outcome of neuromodulation. The therapy-dependent factors for a successful result that we identified were a low number of adverse events and surgical correction of an adverse event. There was no significant difference in the results between patients with complaints of neurogenic origin and idiopathic patients. The number of neurogenic patients (20 patients) was low in comparison with the non-neurogenic group (120 patients). Previous studies by Weil and by Everaert show that psychiatric factors play a role in the firstyear results of SNS. ${ }^{4,19}$

Weil et al. described that patients with a previous history of psychiatric disorder are more prone to loose effect of their implanted neuromodulation system, while Everaert et al. demonstrated a greater chance of different outcomes between the temporary test and definitive implant in patients diagnosed with psychiatric disease. To avoid bias by placebo effect in patients, we established the criterion of a minimum follow-up of one year. 
The rate of surgery for adverse events seems high, compared to other studies. In the literature the percentage of patients needing a reoperation is around $33 \%$, while in our study we found a reoperation rate of $48,3 \%$. $^{4,5,7}$

This suggests a considerable learning curve in patient selection. Throughout the years we select patients more strictly. In the early years potential candidates underwent up to five PNE's when there was doubt if the patient reacted. Now patients get maximal two PNE's before offering them a two-stage tined lead procedure or excluding them from neuromodulation. Furthermore improved temporary lead design made the PNE more reliable. ${ }^{20}$ Another reason for higher reoperation rates is that the used equipment was still under development in the early years of SNS. Fig. 1 shows that the greatest decrease in both adverse events and reoperations is between 1991 and 1992. An important reason for the decrease in adverse events and surgical interventions is the experience that is accumulated, particularly in the first years of the application of sacral neuromodulation. In 1994 both the Itrell-II and a new definitive electrode became available. Fig. 1 shows that in 1994 the adverse events ratio and the reoperation ratio rises. This suggests a learning curve after the introduction of the new techniques.

The years thereafter the ratios drop, showing the benefits of the new techniques. The same phenomenon is seen in 1999 when the Interstim IPG is available and in 2000 when the buttock placement is introduced. Despite the drop in adverse events and reoperation rate a center that embarks on this therapy needs to schedule regular follow-up visits and can expect inbetween visits for troubleshooting patients.

In 1997 Janknegt et al. tried to improve the testing procedure for neuromodulation by testing through the 2-stage procedure. ${ }^{13}$ The long-term efficacy of the 2-stage procedure has been determined by Scheepens et al. ${ }^{11}$ Recently the implant through the 2-stage procedure has been compared with the single-stage procedure in a randomized trial and gives a higher chance of a good result. ${ }^{21}$

Since 2002 a new procedure has been developed for lead implant. ${ }^{22}$ This procedure allows for minimal invasive lead placement under local anesthesia. The procedure takes place on outpatient basis and the tined lead can easily be removed. Therefore two-stage testing through the tined lead has become a valuable addition to the PNE. Experience shows that the rate of patients with positive screening results increases by using the tined lead. 9 This is probably because the tined lead is less susceptible to lead migration and the tined lead allows for longer screening periods.

Since mid-2002 about 50 patients were implanted with the tined lead proce- 
dure in our department. These patients are not included in this study.

We suspect that use of patients' sensory responses during the tined lead implant to guide lead placement gives less chance of failure of the definitive SNS system. Furthermore we expect a decrease in adverse events due to minimal invasive lead implant. These hypotheses have yet to be confirmed in a trial, which is currently ongoing in our clinic.

In 2000 buttock placement of the IPG has been investigated. ${ }^{10}$ The buttock placement reduced average operating time from 2.5 to 1.5 hours because patient turning during surgery was not necessary and the flank incision was not performed. More importantly, postoperative pain, postoperative infections and the need for reoperations for IPG position-related pain were reduced. Despite the lack of a randomized trial the buttock placement has quickly become the standard method of IPG implant.

The occurrence of late failures remains still problematic in SNS. In this study 18 out of 149 patients have been explanted due to loss of efficacy.

This group had more reoperations than the patients that were not explanted. No other predictors were found.

The cause of this phenomenon is still unknown, although we suspect that neuro-plasticity of the micturition center could play an important role.

Currently a trial is ongoing, investigating the effect of bilateral stimulation in patients with therapy failure in our department. Still, more research is needed in this particular group of patients. 


\section{6}

\section{CONCLUSION}

Sacral neuromodulation produces prolonged subjective benefit in a group of patients with highly therapy resistant lower urinary tract symptoms. The decrease in the number of adverse events and reoperations over the study period suggests a considerable learning curve in selecting, implanting and follow-up of patients with neuromodulation. Other contributing factors are the development and implementation of new surgical techniques and the new technical developments as the external patient programmer and new implant hardware. 


\section{7}

\section{ACKNOWLEDGEMENT}

This study was supported by an unrestricted grant from the WAMU Foundation, Maastricht, the Netherlands. 


\section{8}

\section{REFERENCES}

1 Siegel SW. Management of voiding dysfunction with an implantable neuroprosthesis. Urol Clin N Am 1992;19(1): 163-70.

2 Dijkema HE, Weil EH, Mijs PT, Janknegt RA. Neuromodulation of sacral nerves for incontinence and voiding dysfunctions. Clinical results and complications. Eur Urol 1993; 24(1):72-6.

3 Bosch JL, Groen J. Sacral ( $\left.\mathrm{S}_{3}\right)$ segmental nerve stimulation as a treatment for urge incontinence in patients with detrusor instability: results of chronic electrical stimulation using an implantable neural prosthesis. J Urol 1995; 154(2 Pt 1):504-7.

4 Weil EH, Ruiz Cerda JL, Eerdmans PH, Janknegt RA, Van Kerrebroeck PE. Clinical results of sacral neuromodulation for chronic voiding dysfunction using unilateral sacral foramen electrodes. World J Urol 1998;16(5):313-21.

5 Siegel SW, Catanzaro F, Dijkema HE, Elhilali MM, Fowler CJ, Gajewski JB, et al. Long-term results of a multicenter study on sacral nerve stimulation for treatment of urinary urge incontinence, urgency-frequency, and retention. Urology 2000;56(6 Suppl. 1):87-91.

6 Hassouna MM, Siegel SW, Nyeholt AA, Elhilali MM, van Kerrebroeck PE, Das AK, et al. Sacral neuromodulation in the treatment of urgency-frequency symptoms: a multicenter study on efficacy and safety. J Urol 2000;163(6): 1849-54.

7 Janknegt RA, Hassouna MM, Siegel SW, Schmidt RA, Gajewski JB, Rivas DA, et al. Longterm effectiveness of sacral nerve stimulation for refractory urge incontinence. Eur Urol 2001;39(1):101-6.

8 Jonas U, Fowler CJ, Chancellor MB, Elhihali MM, Fall M, Gajewski J, et al. Efficacy of sacral nerve stimulation for urinary retention: results 18 months after implantation. J Urol 2001;165:15-9.

9 Spinelli M, Giardiello G, Arduini A, van den Hombergh U. New percutaneous technique of sacral nerve stimulation has high initial success rate: preliminary results. Eur Urol 2003;43(1):70-4.

10 Scheepens WA, Weil EH, van Koeveringe GA, Rohrmann D, Hedlund HE, Schurch B, et al. Buttock placement of the implantable pulse generator: a new implantation technique for sacral neuromodulation-a multicenter study. Eur Urol 2001;40(4):434-8.

11 Scheepens WA, Van Koeveringe GA, De Bie RA, Weil EH, Van Kerrebroeck PE. Long-term efficacy and safety results of the two-stage implantation technique in sacral neuromodulation. BJU Int 2002;90(9):840-5.

12 Tanagho EA, Schmidt RA. Bladder pacemaker: scientific basis and clinical future. Urology 1982;20(6):614-9.

13 Janknegt RA, Weil EH, Eerdmans PH. Improving neuromodulation technique for re-fractory voiding dysfunctions: two-stage implant. Urology 1997;49(3):358-62. 
14 Spinelli M, Bertapelle P, Cappellano F, Zanollo A, Carone R, Catanzaro F, et al. Chronic sacral neuromodulation in patients with lower urinary tract symptoms: results from a national register. J Urol 2001;166(2):541-5.

15 Jonas U, Fowler CJ, Chancellor MB, Elhilali MM, Fall M, Gajewski JB, et al. Efficacy of sacral nerve stimulation for urinary retention: results 18 months after implantation. J Urol 2001;165(1):15-9.

16 Weil EH, Ruiz Cerda JL, Eerdmans PH, Janknegt RA, Bemelmans BL, van Kerrebroeck PE. Sacral root neuromodulation in the treatment of refractory urinary urge incontinence: a prospective randomized clinical trial. Eur Urol 2000;37(2):161-71.

17 Koldewijn EL, Rosier PF, Meuleman EJ, Koster AM, Debruyne FM, van Kerrebroeck PE. Predictors of success with neuromodulation in lower urinary tract dysfunction: results of trial stimulation in 100 patients. J Urol 1994; $152(6$ Pt 1):2071-5.

18 Scheepens WA, Jongen MMGJ, Nieman FHM, de Bie RA, Weil EHJ, van Kerrebroeck PEV. Predictive factors for sacral neuromodulation in chronic lower urinary tract dysfunction. Urology 2002;60(4):598.

19 Everaert K, De Ridder D, Baert L, Oosterlinck W, Wijndaele JJ. Patient Satisfaction and Complications Following Sacral Nerve Stimulation for Urinary Retention, Urge incontinence and Perineal Pain: A Munticenter Evaluation. Int Urogynaecol J 2000;11:231-6.

20 Weil EH, Ruiz Cerda JL, van den Bogaard AE, van Kerrebroeck PE. Novel test lead designs for sacral nerve stimulation: improved passive fixation in an animal model. J Urol 2000;164(2):551-5.

21 Everaert K, Kerckhaerdt W, Caluwaerdts H, Audenaerdt M, Vereecke H, De Cuypere G, et al. A Prospective Randomized Trial Comparing the 1-Stage with the 2-Stage Implantation of a Pulse Generator in Patients with Pelvic Floor Dysfunction Selected for Sacral Nerve Stimulation. Eur Urol 2004;45:649-54.

22 Spinelli M, Giardiello G, Gerber M, Arduini A, van den Hombergh U, Malaguti S. New sacral neuromodulation lead for percutaneous implantation using local anesthesia: description and first experience. J Urol 2003;170(5): 1905-7. 
CHAPTER 4 


\section{Medium-term Experience of Sacral Neuromodulation by Tined Lead Implantation}

Anco C. Van Voskuilen, Dennis J.A.J. Oerlemans, Ernest H.J. Weil, Ubi van den Hombergh, Philip E.V.A. Van Kerrebroeck BJU INTERNATIONAL (2006) 99, 107-110 


\section{4-1 \\ ABSTRACT}

Objective

To describe patient selection for sacral neuromodulation, also known as Interstim therapy, and the results of tined-lead implantation in the medium term.

\section{Patients and Methods}

In all, 49 patients, 39 with refractory overactive bladder symptoms and 10 with urinary retention, were implanted with the tined lead under local anaesthesia. The mean (SD) test period was 12.4 (5.8) days. Patients were implanted when they had $\mathrm{a} \geq 50 \%$ improvement in voiding diary variables during the test period. The mean follow-up for implanted patients was 15.5 (7.9) months. Changes in voiding variables were compared using a t-test.

\section{Results}

Ten patients had a one-stage and 39 a two-stage implant; of the latter group, 31 (80\%) had a positive response and eight (21\%) did not. In all, 31 patients were included in the follow-up. At the last follow-up, 28 (90\%) patients had a >50\% improvement in diary variables and three (10\%) did not. In 21 patients with urgency symptoms the mean (SD) number of voids decreased from 11.7 (8.9)/day at baseline to $7.3(3.4) /$ day $(\mathrm{P}=0.1)$; the voided volume increased from 160.2 (70.7) $\mathrm{mL}$ to 231.1 (119.5) $\mathrm{mL}(\mathrm{P}=0.001)$; and the number of leakages decreased from 9.5 (8.7) to $3.3(2.2) /$ day $(\mathrm{P}=0.17)$. In the 10 patients with retention, the number of catheterizations decreased from 5.44 (1.6)/day with a volume of 297.6 (76.8) mL, to $1.2(1.7) /$ day and 111.6 (158.1) $\mathrm{mL}$; the mean number of voids increased from 3.7 (3.8)/day with a volume of 123.3 (141.7) $\mathrm{mL}$, to 4.2 (2.4)/day and 248.3 (146.0)mL. There were no significant differences in the variables in the patients with retention. Seven patients had an adverse event. There was one incomplete electrode migration that was treated conservatively. 


\section{Conclusion}

This new minimally invasive approach gives positive results in the medium term. Two-stage testing with the tined lead seems more reliable than the classic percutaneous nerve evaluation. The lead anchoring method seems sufficient for fixing the electrode in the medium term.

Keywords

electric nerve stimulation, LUTS, minimally invasive 


\section{4-2 \\ INTRODUCTION}

Since its introduction about a decade ago, sacral neuromodulation (SNM) is applied in patients with refractory urge incontinence, urgency-frequency and unobstructive urinary retention. ${ }^{1-5}$ Typically, potential candidates are tested with a temporary lead before the implantation of a definitive neuromodulation system is considered. Clinical experience with the test procedure for neuromodulation showed that the temporary electrode is prone to migration during the subchronic test phase. Consequently, and due to the probability of bacterial infections in temporary electrodes that are implanted for a prolonged period, the duration of the subchronic test phase is limited to a maximum of $\approx 1$ week. Moreover, there are some implanted patients who experience inconsistencies between the results of the test period and the results of the definitive implant. In 2003, Spinelli et al.6,7 introduced a new, minimally invasive technique for implanting a definitive electrode. Using this electrode, the duration of the subchronic test phase can be prolonged, and if the test results are insufficient, the electrode can easily be removed. In this article we describe our clinical results with this procedure. 


\section{PATIENTS AND METHODS}

In all, 49 patients (nine men and 40 women, mean age 50.0 years, SD 13.2, range 18-73) with symptoms of overactive bladder (OAB) or urinary retention refractory to conservative therapy, had the new electrode implanted. All patients had a percutaneous nerve evaluation (PNE) with the conventional temporary electrode before the tined-lead implant. Under local anaesthesia, in a minimally invasive procedure, a lead (Model 3889, Medtronic, Minneapolis, MN, USA) was placed in the left or right $\mathrm{S}_{3}$ foramen. ${ }^{6}$ The lead is equipped with silicone tines that allow it to be fixed in the tissue above the sacrum. Local anaesthesia allowed both patients' sensory and motor responses to be used as a guide. The depth of the electrode was controlled by lateral fluoroscopy.

The patients with a positive result from the classic PNE had a one-stage implant. In these patients, the electrode was tunnelled to an incision made at the ipsilateral gluteal area and connected to an extension cable. This extension cable is connected to an implantable pulse generator (IPG, model 3023, Medtronic) inserted into a s.c. pocket in the gluteal area. The one-stage procedure took a mean of $40 \mathrm{~min}$. The patients with inconclusive results of the classic PNE had a two-stage procedure. In these, the lead was tunnelled to a small ipsilateral gluteal incision. After connecting it to an extension cable, the lead was tunnelled to a contralateral exit point and connected to an external stimulator (model 3625, Medtronic). The subchronic phase of the two-stage test took a mean (SD, range) of $12.4(5.8,5-31)$ days. During this phase, patients were asked to keep voiding diaries to record changes in their urinary symptoms. These data were compared to baseline voiding diaries.

Patients with a $\geq 50 \%$ improvement in their relevant urinary symptoms were implanted with an IPG as a second stage. In these, the ipsilateral incision was re-opened and the extension cable was disconnected and removed. A short extension cable was connected to the lead and an IPG was implanted. When the subchronic phase was unsuccessful the lead and extension cable were disconnected and removed. The lead could easily be removed up to a month after 
implantation by applying moderate traction. The first stage of the two-stage procedure took a mean of $30 \mathrm{~min}$, while the second stage took $15 \mathrm{~min}$. All procedures were done under local anaesthesia on an outpatient basis. After implanting the IPG, patients were followed at regular intervals of 6 weeks, 6 months, and yearly thereafter.

Patients were told to contact the clinic when they had problems related to the implanted system. All patients were told to avoid stretching, bowing and bicycling for at least 6 weeks after implantation, after which they were encouraged to resume all activities that they did before the implant. Each patient with loss of efficacy during follow-up had a radiograph taken to check whether there was electrode migration. In June 2005, all implanted patients were asked to complete another set of voiding diaries; these were compared with the baseline diaries. The variables at baseline and at the last follow-up were compared using a t-test. 


\section{4 \\ RESULTS}

Of the 49 patients, $39(80 \%)$ were implanted for OAB symptoms, and $10(20 \%)$ had urinary retention. Ten patients had a positive classic PNE and were implanted in a one-stage procedure. The other 39 patients had an inconclusive PNE, and were re-tested in a two-stage procedure. Reasons for the inconclusive results were: discrepancy in recorded 24-h output between the baseline and test period (13 patients), suspected 108 electrode migration (seven) and technical failure of the PNE (two). One patient with a technical failure had a different anatomy due to spina bifida; in another patient the external stimulator switched off by itself. The other 17 patients with an inconclusive PNE had subjective improvement, while analysis of the voiding diary showed a $<50 \%$ improvement. In the 39 patients who had two-stage testing, 31 (80\%) were eligible for the definitive implant and eight (21\%) were not.

Of all 49 patients, 41 had a definitive implant either in one stage or after twostage testing, and 31 of them were included in the further follow-up. Four of the 10 patients who were not included had a follow-up of $<6$ weeks. A fifth patient with a follow-up of 18.3 months had loss of efficacy after a vaginal wall correction. A sixth patient died 8.9 months after implantation from causes unrelated to neuromodulation, and the seventh patient was explanted for to psychiatric reasons after 13.3 months of follow-up, but had had good results. This patient had hallucinations after the implant and blamed adverse life-events on having the nerve stimulation system. Three patients were lost to follow-up and did not respond to repeated requests to complete an additional diary. These 10 patients were excluded from the analysis.

The mean (SD, range) follow-up of the 31 analysed patients was 15.5 (7.9, 3.232.4) months. At the last follow-up, 28 (90\%) of the patients still had a >50\% improvement in at least one of the relevant voiding diary variables and three $(10 \%)$ patients had a $<50 \%$ improvement. In all implanted patients, the voided volume was increased from 153.0 (86.9) to 234.5 (122.5) $\mathrm{mL}$ at the last follow-up $(\mathrm{P}<0.001)$. Table 1 shows the overall success rates in each patient category. 
In the patients with urge incontinence or urgency-frequency the mean number of voids at baseline was 11.7 (8.9)/day. At last follow-up this decreased to 7.3 (3.4)/day ( $\mathrm{P}=0.1)$. The voided volume increased from 160.2 (70.7) to 231.1 (119.5) $\mathrm{mL}$ at the last follow-up $(\mathrm{P}=0.001)$.

The patients who had urge incontinence had a mean of 9.5 (8.7) incontinent episodes/day at baseline; at the last follow-up this decreased to 3.3 (2.2)/day $(\mathrm{P}=0.17)$. In the 10 patients with retention, the mean number of catheterizations was 5.44 (1.6)/day and the catheterized volume was 297.6 (76.8) $\mathrm{mL}$ at baseline. At last the follow-up the mean number of catheterizations was 1.2 (1.7)/day and the catheterized volume was $111.6(158.1) \mathrm{mL}$. Three of these 10 patients had complete retention at baseline. At the last follow-up, two of these three patients had a normal voiding pattern. Six of the patients with retention no longer catheterize, and the other four catheterize up to a maximum of twice a day. The mean number of voids at baseline was 3.7 (3.8)/day with a volume of 123.3 (141.7) $\mathrm{mL}$. At the last follow-up the number of voids was 4.2 (2.4)/day with a volume of 248.3 (146.0) $\mathrm{mL}$. There were no significant differences in the variables in patients with retention, when analysed by a t-test.

Of 31 patients, seven had a significant adverse event. Two patients had loss of efficacy after non-urological surgery; both patients had positive results after up to two re-programming sessions. Another patient with retention due to an incomplete spinal cord lesion still has no benefit from his system. Two patients complained of pain at the IPG implant site; one had a re-operation under local anaesthesia to reposition the IPG, and the other had a buttock implant of the IPG, while she was dependent on a wheelchair. In this patient the IPG was re-positioned from the gluteal region to the abdominal wall. Both patients are now free of pain and have positive results.

A patient with positive results had an open revision because of troublesome leg stimulation. Investigation showed that the tined lead was placed too deep and stimulated the S2 nerve that runs below the level of the S3 root.

During the follow-up there was one incomplete electrode migration. The patient presented at 5.3 months of follow-up with different sensations and decreased efficacy after a fall. A radiograph image of the sacrum showed that the electrode had migrated by $2-3 \mathrm{~mm}$. The patient was successfully treated by re-programming the stimulator. 
TABLE 1

The overall success rates for patients with urge urinary incontinence and patients with urinary retention.

Patients, \# (\%)

Success

Failure

Total

\begin{tabular}{lccc}
\hline OAB patients & $19(90)$ & $2(10)$ & 21 \\
\hline Retention & 9 & 1 & 10 \\
\hline Total & $28(90)$ & $3(10)$ & 31 \\
\hline
\end{tabular}




\section{5 DISCUSSION}

Successful neuromodulation largely depends on the careful selection of candidates. To date the only means of selecting patients likely to benefit was the classic PNE. ${ }^{8}$ However, the PNE has its limitations. The duration of the subchronic phase of the PNE is limited to a maximum of $\approx 1$ week because of the chance of infection. Furthermore there is a chance of electrode migration. Edlund et al. ${ }^{8}$ reported that eight of 20 patients had a loss of efficacy during the subchronic phase of the PNE; this was probably due to electrode migration, but not in all patients with an insufficient result, as electrode migration could be shown by a sacral radiograph.

In 1997, the two-stage approach was introduced., ${ }^{9,10}$ Testing with a definitive electrode enabled longer testing periods and decreased the chance of a negative test due to electrode migration. Recently Everaert et al. ${ }^{11}$ proved in a randomized trial that testing with the two-stage procedure gave more positive subjective results and a greater improvement in diary variables. As the tined lead can be implanted using a minimally invasive procedure under local anaesthesia, and can easily be removed, testing through the two-stage procedure is easier and applied more often.

The proportion of patients in the present study who had a positive two-stage procedure $(80 \%)$ is higher than the published positive results with the classic PNE, of $30-62 \% .^{8,11-14}$ This comparison is not entirely fair, because some nonresponders were filtered out by the classic PNE, but shows that $\approx 20 \%$ of the negative classic PNEs are false-negative, possibly because the testing period was too short to show clear results, or perhaps because of undetected electrode migration. In a recent study, Kessler et al. ${ }^{15}$ reported a significant difference between $50 \%$ positive responders after $4-7$ days vs $80 \%$ after $\geq 14$ days. These results indicate that the duration of the test period is important in selecting candidates for SNM. It would be interesting to compare the results of the PNE and the twostage tined lead in a randomized prospective trial.

However, in our experience the PNE still has value for initial screening, as it 
is a cost-effective way of establishing whether a patient is a suitable candidate for SNM. Until there are better predictors for the success of SNM, the only tool for patient selection is a temporary test procedure. The PNE keeps the threshold for testing potential candidates low, while filtering unsuitable candidates at an early stage of the assessment. Examples of unsuitable candidates are patients who cannot cope with the testing equipment or the diaries, or those who do not tolerate SNM.

Indications for a two-stage tined lead procedure are a $<50 \%$ symptom reduction during the PNE and/or technical difficulties of the PNE, including suspected lead migration. The patients with urgency had significant improvements at the last follow-up vs baseline. Although patients with retention improved, the variables did not change significantly, but the present group of patients with retention is small and most patients had no complete retention at baseline. The differences in number of voids and voided volume are relatively small, accounting for high $\mathrm{P}$ values. However, the number of catheterizations decreased to a mean of $1 /$ day, and the need to catheterize is bothersome for patients. Of the implanted patients, 90\% maintained a favourable result during the follow-up. This suggests that, in addition to better selection, placing the definitive lead implant under local anaesthesia gives a better chance of lasting positive results than the open implant.

Minimally invasive lead placement is guided by both sensory and motor responses, rather than only motor responses in the open procedure. We suspect that the use of sensory responses in addition to motor responses provides better electrode placement and less chance of complications and therapy failure. This has yet to be confirmed in a study with more patients and a longer follow-up.

One patient had an incomplete electrode migration after a fall. We took sacral radio-graphs in each patient who had loss of efficacy. Despite this rigorous screening policy, there was one migration of $2-3 \mathrm{~mm}$, that was treated conservatively. Kessler et al.described a patient with bilateral electrode migration, who was successfully treated by re-programming the modulator. ${ }^{16}$

In the present study, two patients needed a re-operation due to pain at the implant site. One patient uses a wheelchair, and the IPG implanted in the gluteal area caused discomfort due to the pressure of the wheelchair on the region of the implanted IPG. After repositioning the IPG in the abdominal wall, the patient is free of complaints. Therefore abdominal placement of the IPG should be considered in wheelchair-bound patients. In the other patient with pain at the position of the IPG, we found during re-operation a subcutaneous nerve running through the pocket wall. After re-positioning the IPG away from the nerve 
the patient was free of complaints.

In conclusion, the new minimally invasive approach for SNM is easier to perform than the classic open method. It gives positive results in both the shortand medium-term. Two-stage testing with the tined lead appears to be a more reliable method than the classic PNE because of a prolonged testing period and perhaps because of the lower risk of electrode migration. Therefore testing with a tined lead is a useful alternative in patients with an inconclusive PNE result. As there was only one lead migration during the medium-term follow-up of up to 3 years, it is obvious that that the lead anchoring method is sufficient for fixing the electrode. However, long-term follow-up data on the performance and possible migration of the tined lead are not yet available. 


\section{6}

\section{REFERENCES}

1 Aboseif S, Tamaddon K, Chalfin S, Freedman S, Kaptein J. Sacral neuromodulation as an effective treatment for refractory pelvic floor dysfunction. Urology 2002; 60: 52-6.

2 Aboseif S, Tamaddon K, Chalfin S et al. Sacral neuromodulation in functional urinary retention: an effective way to restore voiding. BJU Int 2002; 90: 662-5.

3 Weil EH, Ruiz-Cerda JL, Eerdmans PH, Janknegt RA, van Kerrebroeck PE. Clinical results of sacral neuromodulation for chronic voiding dysfunction using unilateral sacral foramen electrodes. World J Urol 1998; 16: 313-21.

4 Van Kerrebroeck PE, Van Voskuilen AC, Lycklama A et al. Long-term results of interstim therapy for voiding disorders demonstrate sustained efficacy and acceptable safety profile. Eur Urol Suppl 2004; 3: 170, Abstract 670.

5 Siegel SW, Catanzaro HE, Dijkema HE et al. Long-term results of a multicenter study on sacral nerve stimulation for treatment of urinary urge incontinence, urgency-frequency, and retention. Urology 2000; 56 (Suppl. 6): 87-91.

6 Spinelli M, Giardiello G, Gerber M, Arduini A, van den Hombergh U, Malaguti S. New sacral neuromodulation lead for percutaneous implantation using local anesthesia: description and first experience. J Urol 2003; 170: 1905-7 7 Spinelli M, Giardiello G, Arduini A, van den Hombergh U. New percutaneous technique of sacral nerve stimulation has high initial success rate: preliminary results. Eur Urol 2003; 43: 70-4.

8 Edlund C, Hellstrom M, Peeker R, Fall M. First Scandinavian experience of electrical sacral nerve stimulation in the treatment of the overactive bladder. Scand J Urol Nephrol 2000; 34: $366-76$.

9 Janknegt RA, Weil EH, Eerdmans PH. Improving neuromodulation technique for refractory voiding dysfunctions: two-stage implant. Urology 1997; 49: 358-62.

10 Scheepens WA, Van Koeveringe GA, De Bie RA, Weil EH, Van Kerrebroeck PE. Long-term efficacy and safety results of the two-stage implantation technique in sacral neuromodulation. BJU Int 2002; 90: 840-5.

11 Everaert K, Kerckhaert W, Caluwaerts $\mathrm{H}$ et al. A prospective randomized trial comparing the 1-stage with the 2-stage implantation of a pulse generator in patients with pelvic floor dysfunction selected for sacral nerve stimulation. Eur Urol 2004; 45: 649-54.

12 Bosch JL, Groen J. Sacral nerve neuromodulation in the treatment of patients with refractory motor urge incontinence: long-term results of a prospective longitudinal study. J Urol 2000; 163: 1219-22.

13 Hassouna MM, Siegel SW, Nyeholt AA et al. Sacral neuromodulation in the treatment of urgency-frequency symptoms: a multicenter study on efficacy and safety. J Urol 2000; 163: 1849-54. 
14 Weil EH, Ruiz-Cerda JL, Eerdmans PH, Janknegt RA, Bemelmans BL, van Kerrebroeck PE. Sacral root neuromodulation in the treatment of refractory urinary urge incontinence: a prospective randomized clinical trial. Eur Urol 2000; 37: 161-71.

15 Kessler TM, Madersbacher H, Kiss G. Prolonged sacral neuromodulation testing using permanent leads: a more reliable patient selection method? Eur Urol 2005; 47: 660-5.

16 Kessler TM, Madersbacher H, Kiss G. Bilateral migration of sacral neuromodulation tined leads in a thin patient. J Urol 2005; 173: 153-4. 
CHAPTER 5 


\section{Is on-Demand Sacral Neuromodulation in Patients with $O A B$ Syndrome a Feasible Therapy Regime?}

Dennis J.A.J. Oerlemans, Anco C. van Voskuilen, Tom A.T. Marcelissen, Ernest H.J. Weil, Rob A. de Bie, Philip E.V.A. Van Kerrebroeck

Neurourology and Urodynamics 30:1493-1496 (2011) 


\section{1}

\section{ABSTRACT}

\section{Introduction}

Sacral neuromodulation (SNM) of the lower urinary tract has proven to be safe and effective in patients with complaints of $\mathrm{OAB}$ syndrome who are not responding to conservative therapy. After five years of treatment the implanted system is still effective in $56-71 \%$ of patients. The loss of effect could be caused by adaptation of the nerve system to prolonged stimulation of the sacral nerves.

\section{Materials and Methods}

We set up a pilot intervention study. After a run-in period of 2 weeks patients were randomized into two groups: one group with on-demand neuromodulation (intervention group) and one group with continuous neuromodulation (control group). Patients in the intervention group were instructed to switch their INS off by default and to switch it on again when they felt recurrent symptoms, patients in the control group were asked to use their system as normally.

\section{Results}

After 2 weeks 10 out of 16 subjects reported a comparable symptom score during on-demand use of their neuromodulation system. Patients appreciated the comfort of being self-determent in the need for therapy.

\section{Conclusions}

Possible benefits for patients could be: more autonomy, longer battery life of the implanted INS, decreasing the chance of adaptation by the nervous system. 


\section{5-2}

\section{INTRODUCTION}

Sacral neuromodulation (SNM) of the lower urinary tract has proven to be safe and effective in patients with complaints of OAB syndrome who are not responding to conservative therapy. ${ }^{1,2}$ SNM treatment is also used in patients with non-obstructive urinary retention and as an off-label treatment in patients with interstitial cystitis. ${ }^{3}$ After 5 years of treatment the implanted system is still effective in $56-71 \%$ of patients. ${ }^{4}$ The system consists of an implanted lead, stimulating the sacral nerves through one of the sacral foramina (preferably $\mathrm{S}_{3}$ ), connected to an implantable neurostimulator (INS). Typical battery life is 8-10 years; depending on the parameters used for stimulation. The expected lifespan of the recently introduced 'Inter-stim II' lies around 3-5 years when used $24 \mathrm{hr} /$ day.

Most of the patients with an implanted neuromodulation system for urinary complaints receive continuous neuromodulation $24 \mathrm{hr}$ a day. However, they are instructed to turn the INS off at certain moments, for example, when driving a car or when working with heavy machinery. This is to prevent accidents caused by reactions to sudden changes in the electrical current.

Until now evidence is lacking whether it is necessary to apply $24 \mathrm{hr}$ of neuromodulation per day or if discontinuation of neuromodulation is possible for a short period of time.

In early trials on SNM the effect of prolonged discontinuation of neuromodulation for at least $72 \mathrm{hr}$ showed return to baseline of voiding symptoms in all patients. This was reversible in most patients by turning the neuromodulator on again. ${ }^{1,5}$

The working mechanism of SNM is not fully understood but seems to involve modulation of spinal cord reflexes and brain networks by peripheral afferents, rather than direct stimulation of the motor response of the detrusor muscle. SNM is proposed to activate or 'reset' somatic afferent inputs that play a pivotal role in the modulation of sensory processing and micturition reflex pathways in the spinal cord ${ }^{6,7}$ Furthermore, there are indications that SNM influences brain 
areas that are involved in detrusor hyperactivity, awareness of bladder filling, the urge to void and the timing of micturition..$^{8,9}$

With on-demand rather than continuous neuromodulation, the physiological situation is more adequately approximated, since in the normal physiological situation there is no continuous tonic afferent information coming from afferents to the dorsal sacral horn. Therefore, patients are expected to have a lower risk of developing side effects of SNM such as gastrointestinal complaints, pain, or menstrual cycle changes.

Clinical experience as well as the literature on SNM has shown that a certain percentage of implanted patients stop having benefit from their implanted system after a few years, despite adequate neuromodulation. A suggested hypothesis for this phenomenon is adaptation of the nerve system to prolonged stimulation of the sacral nerves. ${ }^{10}$ It could be expected that on-demand neuromodulation postpones or diminishes this effect.

The objective of this prospective pilot study was to investigate whether the on-demand use of SNM is a feasible therapy regime, therewith mimicking the normal physiological situation as well as diminishing the need for frequent replacement of the INS. 


\section{5-3 \\ PATIENTS AND METHODS}

This study was set up as a pilot intervention study. Patients who were treated with SNM for symptoms of urgency incontinence or urgency-frequency syndrome refractory to conservative therapy were included. Patients were considered eligible for the study when they had a functioning neuromodulation system with unchanged stimulation settings for at least 6 months. All participants were implanted with a system that includes a patient programmer, which gives the possibility to deactivate the system (Medtronic Interstim model 3023 and Model 3031A patient programmer, Medtronic, Minneapolis, MN). Patients with relevant co-morbidity were excluded (e.g. urinary tract infection, non-functioning system, and malignancy of the urinary tract).

For this study a hybrid design was followed, based on previous work by Pocock. ${ }^{11}$ Instead of a 50:50 allocation of patients, $25 \%$ of the participants were randomized to the control group, permitting $75 \%$ to be assigned to the new intervention. We started with all patients and follow them for 2 weeks to establish a firm baseline (and the knowledge that with continous SNM there will be maximum 'on' time and very likely no change in symptoms). After 2 weeks patients were randomized to the on-demand group, while five stayed in the continuous (control) group. At the end of study, the data from the controls were compared to the baseline group and showed stability. This allowed us to combine all controls and compare them to the on-demand group. This enabled us to perform a transversal comparison with a minimum of patients. The main criteria for such an approach (rather recent included patients, the same procedure, investigator, and identical outcome assessments) were all met in this trial.

All included patients signed an informed consent and were asked to register a voiding diary for 2 weeks (baseline) and to fill out a Urologic Measurement Questionnaire (UMQ). This UMQ measures subjective urologic complaints in patients. This UMQ consists of seven questions on urologic complaints; a score of 1 (much worse) to 5 (much better) can be given to each of the questions (Appendix 1). The questionnaire is previously used in other studies regarding SNM 
for the measurement of subjective complaints. ${ }^{12}$ This questionnaire was chosen because it has been used in our practice since the start of our neuromodulation database and appeared to be very useful in clinical practice and follow-up of individual patients. Although it is not a validated questionnaire, it gives a comparable scale of subjective complaints. We consider the data of interest to the clinician as they give an impression of the magnitude of change.

The total study duration was 4 weeks and started with a run in period of 2 weeks: in this period all subjects registered a voiding diary (baseline symptoms). At the end a urogical questionnaire had to be filled out. After the run-in period of 2 weeks all patients were randomized into two groups: one group with on-demand neuromodulation (intervention group) and one group with continuous neuromodulation (control group). For the remaining 2 weeks, the patients in the intervention group were instructed to switch their INS off by default and to switch it on again when they felt recurrent symptoms. So when they experienced more urgency symptoms or incontinence they turned their INS on. When symptoms had disappeared they could turn the INS off again. The patients in the control group were asked to use their system as normally: this means they were not allowed to change settings during the trial.

At the end of this period, subjects were asked to fill out the UMQ again (Appendix 2).

With a voiding diary urinary complaints as well as the on and off-hours were registered for both groups. The following voiding diary parameters were evaluated: number of voids $/ 24 \mathrm{hr}$, voided volume/void, number of leakages $/ 24 \mathrm{hr}$, number of pads $/ 24 \mathrm{hr}$, and the degree of urgency.

On-demand neuromodulation was considered successful when patients were able to switch the INS off for at least $4 \mathrm{hr} /$ day, without worsening of urinary symptoms recorded with voiding diaries and UMQ. This cutoff point was set at $4 \mathrm{hr}$ because this was considered a time span that could give a relevant change in battery lifespan.

Institutional review board approval was obtained for this study. All included patients signed an informed consent. Data analysis was performed using Statistical Package for the Social Science version 12.0 (SPSS Inc., Chicago, IL). 
TABLE 1

Baseline symptoms for both groups with continuous neuromodulation

\begin{tabular}{lcccc}
\hline & $\begin{array}{c}\text { Pads/ } \\
\text { day }\end{array}$ & $\begin{array}{c}\text { Volume/ } \\
\text { void }\end{array}$ & $\begin{array}{c}\text { \# Micturition/ } \\
\text { day }\end{array}$ & $\begin{array}{c}\text { \# Leakages/ } \\
\text { day }\end{array}$ \\
\hline On-demand & 0.9 & $131 \mathrm{ml}$ & 8.7 & 2.9 \\
\hline Control & 1.1 & $202 \mathrm{ml}$ & 7.3 & 2.8 \\
\hline
\end{tabular}




\section{4 \\ RESULTS}

In total 26 patients were included in this study. Five patients were excluded from analysis because they did not fill out the voiding diaries correctly.

Complete voiding diaries were obtained of the remaining 21 patients, who were randomized into two groups. Five patients were randomized into the control group and 16 into the on-demand group. In the on-demand group there were 2 men and 14 women, in the control group only women. The mean age of these patients was 53.6 years. All patients had their neuromodulation system implanted between 1992 and 2004 for urgency incontinence. By the time they were participating in this study an Interstim model 3023 was implanted. In all patients the INS configuration was unchanged in the last half year and all subjects were capable of using their patient programmers correctly.

Baseline symptoms for both groups with continuous neuromodulation are shown in Table 1. There were no statistical differences in baseline parameters between the two groups.

In the control group no differences were observed between the results of voiding diaries and UMQ during the first 2 weeks (baseline) and the second 2 weeks of this study. In the intervention group 10 of 16 subjects reported a comparable symptom score (no worsening) during on-demand use of their neuromodulation system. They had a mean off-time of $12.4 \mathrm{hr}$ a day.

Table 2 shows the mean off-times in hours a day for the intervention group. Worsening was measured based on the results of the voiding diaries.

Table 3 shows the different parameters for the subjects of the intervention group.

After the intervention period all participating subjects filled out a UMQ. With this questionnaire subjective differences between both periods were measured with seven questions. (min-max score 7-35). A score below 17 was considered as a worsening in subjective measurements (Table 4). 
TABLE 2

Number of patients with off-hours/day during intervention

Modulator off (hrs)

Worsening

No worsening

Total

\begin{tabular}{llll}
\hline $4-14$ & 0 & 2 & 2 \\
\hline $14-24$ & 6 & 8 & 14 \\
\hline Total & 6 & 10 & 16 \\
\hline
\end{tabular}

TABLE 3

Micturition parameters during intervention period (mean off-time per day in hours)

No. Pts.

No. Pts.

Increase (not successful) No increase (succesful)

\begin{tabular}{lcc}
\hline Voids/24 hr & $4(10 \mathrm{hr})$ & $12(18 \mathrm{hr})$ \\
\hline Leakage episodes & $6(8,4 \mathrm{hr})$ & $10(12,4 \mathrm{hr})$ \\
\hline Pad use & $6(8,4 \mathrm{hr})$ & $10(12,4 \mathrm{hr})$ \\
\hline Severity of leakage & $4(10 \mathrm{hr})$ & $12(18 \mathrm{hr})$ \\
\hline
\end{tabular}

TABLE 4

Scores for subjective measurements (urologic measurement questionnaire)

$\begin{array}{llll}\text { Subjective change } & \text { Control } & \text { On-demand } & \text { Total }\end{array}$

\begin{tabular}{llll}
\hline Worsening $(</=17)$ & 0 & 8 & 8 \\
\hline No worsening $(>17)$ & 5 & 8 & 13 \\
\hline Total & 5 & 16 & 21 \\
\hline
\end{tabular}




\section{5}

\section{DISCUSSION}

SNM is an approved treatment for different challenging urological conditions. ${ }^{1-3}$ Unfortunately some patients tend to loose their initial effect over the years. Long-term multicenter results show an efficacy off $56-71 \%$ for the different relevant parameters at 5 years. ${ }^{4}$ Possible explanations for failures during use of SNM include the potential placebo effect of test stimulation, insufficient test stimulation sensitivity before implantation, and inadequate patient selection. These explanations are supported by a good correlation between the 1- and 5 -year success rates.

Adaptation of the nervous system, although never clearly studied, is another proposed mechanism for the loss of effect on the long-term for prolonged stimulation of the sacral nerves. On-demand neuromodulation might be an ideal way of stimulation to prevent this phenomenon.

The results in Table I show that 14 out of 16 patients in the intervention group had their INS turned off for at least $14 \mathrm{hr}$ a day. 10 out of 16 patients could turn their INS off without worsening of complaints during this period, and 8 of these patients could do that for at least $14 \mathrm{hr}$.

In this study, $62.5 \%$ of the patients in the intervention group showed no increase in pad use and/or number of leakage episodes a day (Table 2). Looking to the number of voids in a day and the severity of leakage $75 \%$ of the intervention group had no increase in symptoms. This means that for an average group of patients treated with SNM for urgency incontinence almost $2 / 3$ could be treated with on-demand neuromodulation.

In the intervention group $50 \%$ of patients experienced worsening in subjective symptoms. This result can be explained by the way our patients were instructed. They were told to the turn the INS on during the intervention period when they felt recurring symptoms. As expected the patients in the control group experienced no difference (Table 3 ).

Another method of non-continuous neuromodulation is stimulation by cycling mode. This is possible with all INS nowadays used for SNM therapy. With 
this setting the INS turns itself on and off in a preprogrammed cycle. For example the stimulation can be programmed on during 10 second then off for 5 sec. Disadvantages of this method compared to on-demand neuromodulation is that a patient experiences this on/off event every time. Most patients in our practice reported discomfort during this cycling mode stimulation. A second disadvantage is that it is not the patient itself that determines the need for therapy.

When patients were asked how they experienced on-demand neuromodulation, many patients appreciated the comfort of being self-determent in the need for therapy. They felt less dependent on their neuromodulation system. However, we did not directly measure the influence of this reported effect on quality of life. While an improvement in quality of life may be appreciated by some, those who are satisfied with continuous stimulation such that they no longer think about their prior voiding complaints, may view intermittent stimulation as an interruption and an impediment to their quality of life.

The long-term effects of on-demand neuromodulation were not studied in this pilot. Therefore long-term studies are necessary to determine if on-demand neuromodulation can prevent adaptation of the nervous system. 


\section{$5 \cdot 6$}

\section{CONCLUSION}

According to this pilot study, on-demand neuromodulation is a feasible therapy regime in the majority of patients with urgency incontinence successfully treated with SNM therapy. Possible benefits for patients could be:

- More autonomy; it allows patient to confidently turn of their SNM system without losing efficacy.

- Longer battery life of the implanted INS.

- Decreasing the risk of adaptation by the nervous system.

Nevertheless, larger, randomized controlled studies with longer follow-up are needed and should include quality of life measurements as well as a more detailed data on battery life improvement. 


\section{$5 \cdot 7$}

\section{ACKNOWLEDGEMENTS}

This study was funded by the WAMU Foundation Maastricht. We would like to thank Q. de Waard for his work. 


\section{8}

\section{APPENDIX 1}

\section{Urologic Measurements Questionnaire}

1. Do you think that voiding in general goes better?

No, it's much worse/no it's worse/no difference/yes, some improvement/yes a lot better.

2. Do you think that your flow has improved?

No, it's much worse/no it's worse/no difference/yes, some improvement/yes a lot better.

3. Does your bladder feel emptier?

No, it feels much fuller/no, it feels a bit fuller/no difference/yes, it's emptier/ yes, it's much emptier.

4. Do you think you are drier?

No, much wetter/no, a bit wetter/ no difference/yes, a bit drier/yes, much drier.

5. Have your urge-sensations changed?

Clearly worsened/a bit worse/no difference/a bit less/clearly diminished.

6. Has your voiding frequency changed?

No, I had to go more often/no, I had to go a bit more often/no difference/Yes, I had to go somewhat less often/yes, I clearly had to go less often.

7. Do you have more control over your bladder?

No, it's worse/No, it's somewhat worse/no difference/yes, it's better/yes, it's much better.

Score 1 (much worse) -5 (much better) 


\section{9}

\section{APPENDIX 2}

Course and Timing of the Study

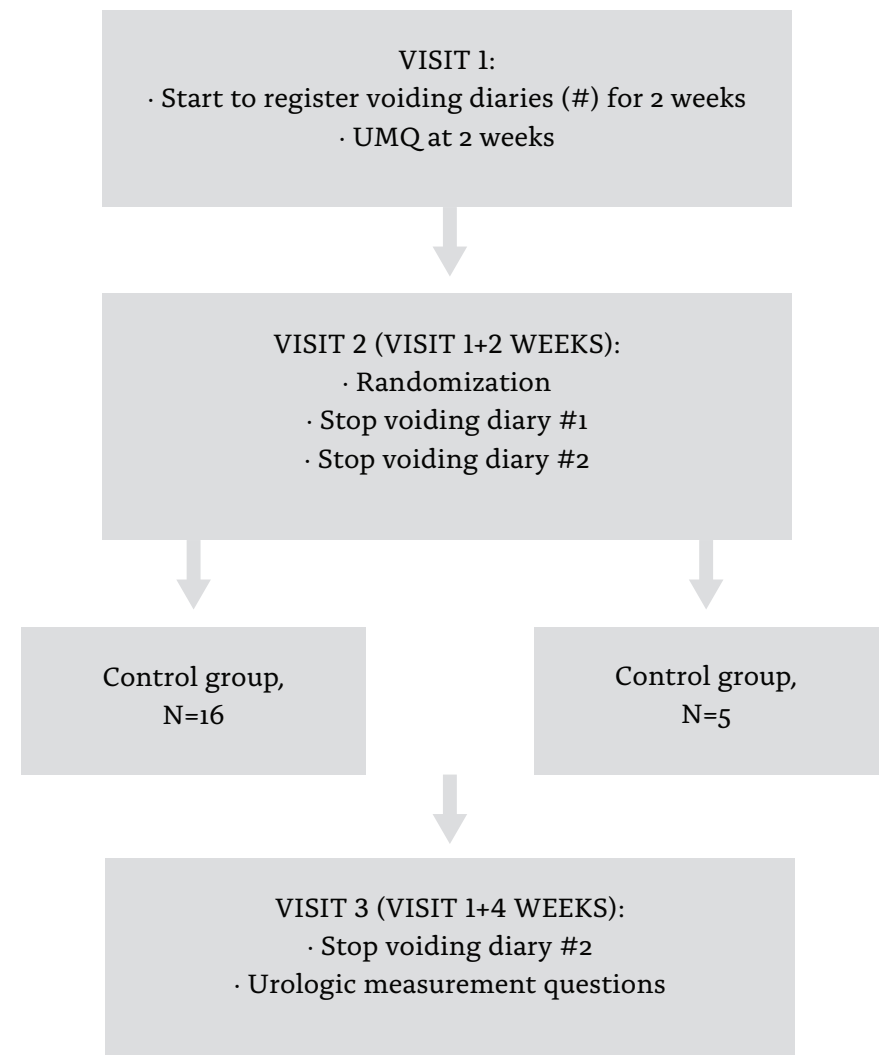




\section{5-10 \\ REFERENCES}

1 Hassouna MM, Siegel SW, Nyeholt AA, et al. Sacral neuromodulation in the treatment of urgency-frequency symptoms: A multicenter study on efficacy and safety. J Urol 2000;163:1849.

2 Schmidt RA, Jonas U, Oleson KA, et al. Sacral nerve stimulation for treatment of refractory urinary urge incontinence. Sacral Nerve Stimulation Study Group. J Urol 1999;162:352.

3 Jonas $\mathrm{U}$, Fowler CJ, Chancellor MB, et al. Efficacy of sacral nerve stimulation for urinary retention: Results 18 months after implantation. J Urol 2001; 165:15.

4 van Kerrebroeck PE, van Voskuilen AC, Heesakkers JP, et al. Results of sacral neuromodulation therapy for urinary voiding dysfunction: Outcomes of a prospective, worldwide clinical study. J Urol 2007;178: 2029.

5 Weil EH, Ruiz-Cerda JL, Eerdmans PH, et al. Sacral root neuromodulation in the treatment of refractory urinary urge incontinence: A prospective randomized clinical trial. Eur Urol 2000;37:161.

6 Fowler CJ, Swinn MJ, Goodwin RJ, et al. Studies of the latency of pelvic floor contraction during peripheral nerve evaluation show that the muscle response is reflexly mediated. J Urol 2000;163:881.

7 Vodusek DB, Light JK, Libby JM. Detrusor inhibition induced by stimulation of pudendal nerve afferents. Neurourol Urodyn 1986;5:381.

8 Dasgupta R, Critchley HD, Dolan RJ, et al. Changes in brain activity following sacral neuromodulation for urinary retention. J Urol 2005;174:2268.

9 Blok BF, Groen J, Bosch JL, et al. Different brain effects during chronic and acute sacral neuromodulation in urge incontinent patients with implanted neuro-stimulators. BJU Int 2006;98:1238-43.

10 Agnew WF, McCreery DB, Yuen TG, et al. Evolution and resolution of stimulation-induced axonal injury in peripheral nerve. Muscle Nerve 1999;22:1393.

11 Pocock SJ. The combination of randomized and historical controls in clinical trials. J Chronic Dis 1976;29:175-88.

12 Scheepens WA, de Bie RA, Weil EH, et al. Unilateral versus bilateral sacralneuromodulation in patients with chronic voiding dysfunction. J Urol 2002;168:2046-50. 
CHAPTER 6 


\section{Sacral Neuromodulation in Patients with Idiopathic Overactive Bladder after Initial Botulinum Toxin Therapy}

Dennis J.A.J. Oerlemans*, Martijn A.C. Smits*, Tom A.T. Marcelissen, Philip E.V.A. Van Kerrebroeck^, Stefan G.G. De Wachter ${ }^{\wedge}$

${ }^{*}$ Equal study contribution

$\wedge$ Equal study contribution

The Journal of Urology 190: 2148-2152 (2013) 


\section{1 \\ ABSTRACT}

\section{Purpose}

We evaluated whether patients with overactive bladder and incontinence who discontinued intravesical botulinum toxin therapy can be successfully treated with sacral neuromodulation.

\section{Materials and methods}

All patients who were referred to our center after discontinuation of botulinum toxin-A between 2005 and 2010 were included in this observational study. All patients underwent test stimulation with sacral neuromodulation and were evaluated with voiding diaries. Success was defined as more than $50 \%$ improvement in leakage episodes. Successful test stimulation was subsequently followed by a definitive implant. Patient satisfaction with sacral neuromodulation therapy was evaluated 1 year after the definitive implant.

\section{Results}

A total of 20 patients were included in the study. Of these patients 17 (85\%) had discontinued botulinum toxin-A because of lack of efficacy and 3 had been treated successfully with botulinum toxin-A but requested a more permanent solution. The mean interval between the botulinum toxin-A and the sacral neuromodulation test stimulation was 23 months. In 14 patients (70\%) the test stimulation was successful and they received a definitive implant. Of the 14 patients 5 even showed a decrease of greater than 90\% in leakage episodes. One year after implantation 11 patients (79\%) were satisfied with the sacral neuromodulation treatment.

\section{Conclusions}

Despite the small sample size, this study indicates that patients who are dissatisfied with or in whom botulinum toxin-A treatment fails can respond successfully to sacral neuromodulation. The success rate of the test stimulation was 
comparable to that of patients who have never been treated with botulinum toxin-A. The 1-year satisfaction rate was comparable that of patients without a history of botulinum toxin-A treatment. 


\section{6-2 \\ INTRODUCTION}

Overactive bladder is defined as lower urinary tract symptoms of urgency with or without urinary incontinence (OAB-wet and OAB-dry), usually with frequency and nocturia. It can be caused by detrusor overactivity of neurogenic or idiopathic origin. The prevalence is estimated up to $17 \%$ in the adult population and increases with age. First line conservative treatment consists of lifestyle modifications, pelvic floor physiotherapy and anticholinergic medications. These conservative treatments do not always lead to sufficient improvement of complaints and are often associated with considerable side effects resulting in limited compliance and treatment failure. Intravesical injections with botulinum toxin and sacral neuromodulation are minimally invasive and reversible second line treatment alternatives which have gained wide acceptance in the treatment of OAB. ${ }^{1}$

BoNTA treatment has largely been investigated in patients with neurogenic detrusor activity for which there is now unanimously a high level of recommendation and approval of the U.S. Food and Drug Administration (FDA). The use of BoNTA treatment in patients with idiopathic detrusor overactivity is currently studied. The FDA has recently approved the treatment for idiopathic detrusor overactivity and it has been adapted in the clinical practice guidelines and is performed on a large scale. Recent placebo controlled randomized control trials have shown BoNTA treatment to be effective for idiopathic detrusor overactivity, with success rates ranging from 40 to $80 \%$ depending on the dose used., ${ }^{1,2}$

BoNTA treatment is regarded overall safe and well tolerated when injected in the bladder wall with current doses and techniques. The most common side effect of the treatment is the increase in PVR resulting in possible need for CISC and urinary tract infections. The need for CISC after BoNTA treatment has been reported in a range from $16 \%$ to $42 \% .3,4$ Remarkably, up to $37 \%$ of the patients discontinue the treatment after the first 2 injections. This is mainly due to lack of efficacy, presence of adverse events such as post-void residual that needs clean intermittent catheterization or the desire for a more permanent 
solution (without the need for repeated treatments). ${ }^{5}$ If BoNTA treatment fails or when patients are dissatisfied with the result, sacral neuromodulation is the only other minimal invasive alternative. In this study we determined whether patients who have been unsuccessfully treated with BoNTA treatment can be successfully treated with SNM. 


\section{3}

\section{MATERIALS AND METHODS}

In this single center observational study we included all patients with idiopathic OAB-wet that were referred between 2005 and 2010. All these patients had stopped BoNTA treatment due to dissatisfaction and had recurrent or persistent symptoms.

History taking and a 3-day voiding diary assessed the complaints of OAB. The voiding diary was used as baseline. Physical examination and urinalysis was used to rule out other conditions. Urodynamics were performed in all patients to confirm the diagnosis of detrusor overactivity and to exclude other diagnosis.

All patients underwent SNM test stimulation with use of the PNE test. In all patients there was a minimum interval of 6 months between the last BoNTA injections and this SNM test stimulation, to prevent bias caused by botulinum toxin at SNM screening. This 1-week test stimulation was subsequently followed by a 1 or 2-stage TLP. During the test stimulation period, a 3-day voiding diary was again filled out to evaluate the effect of SNM. The test stimulation was evaluated by comparing the data of the voiding diary filled out during the test stimulation to the baseline voiding diary.

Primary outcome measure was improvement in leakage, defined as greater than $50 \%$ improvement in episodes of leakage and severity of leakage (subjective on a scale from 1 to 4 ). Improvement in frequency and/or urgency was stated as the secondary outcome, for which success was defined as greater than $50 \%$ improvement in frequency and urgency. In case of successful PNE, it was followed by a 1-stage TLP with definitive placement of an implantable neurostimulator (InterStim ${ }^{\circledR}$ I or InterStim II, Medtronic Inc). In case of an unsuccessful PNE test stimulation, a first stage TLP was performed. When the first stage was successful, the second stage of the TLP consisted of a definitive placement of an implantable pulse generator. If unsuccessful the first stage was surgically removed. Several pulse generator settings were used, preferably unipolar electrode selection, frequency of $10 \mathrm{~Hz}$, the pulse width 210 us and the amplitude 
just above sensation threshold (preferably lower than 2.0 V).

At routine annual control at the outpatient clinic the patients who received the definitive SNM implant were asked about their satisfaction. The patients who were not eligible for SNM underwent a telephone interview about the course of their treatment after SNM test stimulation failure. The data processing of this study was performed with IBM SPSS ${ }^{\circledR}$ statistics, version 18. 


\section{4 \\ RESULTS}

A total of 20 patients ( 16 female), mean age 56 years (range 37 to 82 ) were screened for SNM. Table 1 gives an overview of all patient data.

Seventeen (85\%) patients had stopped BoNTA treatment, because of lack of efficacy during the first or subsequent treatments. Three patients had experienced successful BoNTA treatment, but were dissatisfied because of the temporary relief and need of repeat injections and opted to be screened for treatment with SNM. Four (20\%) of the patients unsatisfied with BoNTA reported the need for performing CISC during BoNTA treatment, although they did not experience any effect on urgency urinary incontinence. Because of increased PVR (greater than $150 \mathrm{cc}$ ) 3 of the 4 were still performing CISC, at the time of SNM test stimulation.

The number of injection sessions per patient ranged from 1 to 4 subsequent BoNTA treatments, dosages varying from 100 to 300 units onabotulinumtoxin $A$ in up to 500 units abobotulinumtoxin A. The mean interval between the (last) BoNTA treatment and the SNM test stimulation was 23 months (minimum 7, maximum 53). Ten patients (50\%) responded successfully to PNE and another $4(20 \%)$ in whom PNE failed experienced a secondary successful first stage TLP. The total success rate was $70 \%$ ( 14 of 20 ). Voiding diaries during test stimulation showed greater than $50 \%$ decrease of episodes (mean $75 \%$ ) with a mean decrease in severity of leakage of $53 \%$ when comparing the diaries of the test stimulation to the baseline voiding diaries. Five (25\%) patients even showed a decrease of greater than $90 \%$ in leakage episodes. The changes in frequency varied from an increase of $15 \%$ to a decrease of $62 \%$. Of the 3 patients who still had to perform CISC before SNM test stimulation (table 1, patients 1, 9 and 17) 2 were voiding to completion during SNM test stimulation (patients 1 and 9). Table 2 shows an overview of the screening results. No difference is shown between success rates in patients who received repeated injections (patients 3, 5, 6, 9, 10 and 13) and those who only had a single injection.

All 14 patients underwent implantation of an internal pulse generator (In- 
terStim I or InterStim II). One year after implantation 11 of the 14 patients (79\%) were subjectively satisfied with the SNM treatment. All 3 patients who were dissatisfied with SNM experienced a relapse of incontinence that could not be controlled by changing the parameter settings.

In 5 patients the PNE and the secondary first stage TLP were unsuccessful. One patient refused a secondary first stage TLP after failure of the PNE. These 6 patients who did not experience a sufficient effect during the PNE and TLP 1st stage test stimulation were questioned about their treatment course after this test failure and 4 did not receive any other treatment and are still experiencing symptoms of OAB. Two patients were lost to follow-up.

TABLE 1

Supplementary table: overview patient data

\begin{tabular}{|c|c|c|c|c|c|}
\hline $\begin{array}{l}\text { Patient } \\
\text { ID }\end{array}$ & Sex & $\begin{array}{l}\text { Age } \\
\text { (yrs) }\end{array}$ & $\begin{array}{c}\text { No. of } \\
\text { BoNTA }\end{array}$ & $\begin{array}{l}\text { Efficacy } \\
\text { BoNTA }\end{array}$ & $\begin{array}{c}\text { Reason discontinuation } \\
\text { BoNTA }\end{array}$ \\
\hline 1 & Female & 37 & 1 & No effect & No effect, PVR \\
\hline 2 & Female & 54 & 1 & No effect & No effect \\
\hline 3 & Female & 82 & 4 & Dry & Repeat treatment \\
\hline 4 & Female & 70 & 1 & No effect & No effect \\
\hline 5 & Female & 54 & 2 & Dry & Repeat treatment \\
\hline 6 & Female & 53 & 2 & No effect & No effect \\
\hline$\underline{7}$ & Male & 42 & 1 & Dry & Repeat treatment \\
\hline 8 & Male & 60 & 1 & No effect & No effect \\
\hline 9 & Female & 66 & 2 & No effect & PVR, persist incontinence \\
\hline 10 & Male & 78 & 2 & No effect & No effect \\
\hline 11 & Female & 44 & 1 & No effect & No effect \\
\hline 12 & Female & 47 & 1 & No effect & No effect \\
\hline 13 & Male & 67 & 1 & No effect & No effect \\
\hline 14 & Female & 55 & 1 & No effect & No effect \\
\hline 15 & Female & 49 & 1 & No effect & No effect, PVR \\
\hline 16 & Female & 66 & 3 & Dry & Repeat treatment, no effect \\
\hline 17 & Female & 68 & 1 & No effect & PVR, persist incontinence \\
\hline 18 & Female & 46 & 1 & No effect & No effect \\
\hline 19 & Female & 40 & 1 & No effect & No effect \\
\hline 20 & Female & 54 & 1 & No effect & No effect \\
\hline
\end{tabular}


TABLE 2

Results of screening

\begin{tabular}{lccccc} 
Patient & Mos BoN- & Test stimula- & Leakage im- & Frequency im- & 1-yr \\
ID & TA-SNM & tion result & provement (\%) & provement (\%) & satisfaction \\
\hline
\end{tabular}

Succesful test stimulation with definitive implant performed:

\begin{tabular}{|c|c|c|c|c|c|}
\hline 1 & 18 & PNE succesful & Greater than 50 & Less than 50 & Unsatisfied \\
\hline 2 & 25 & PNE succesful & Greater than 50 & Less than 50 & Satisfied \\
\hline 3 & 17 & PNE succesful & Greater than 50 & Greater than 50 & Satisfied \\
\hline 4 & 8 & PNE succesful & Greater than 50 & Less than 50 & Satisfied \\
\hline 5 & 41 & PNE succesful & Greater than 50 & Greater than 50 & Unsatisfied \\
\hline 6 & 11 & PNE succesful & Greater than 90 & Greater than 50 & Satisfied \\
\hline 7 & 21 & PNE succesful & Greater than 90 & Less than 50 & Satisfied \\
\hline 8 & 7 & PNE succesful & Greater than 90 & Less than 50 & Satisfied \\
\hline 9 & 17 & PNE succesful & Greater than 50 & Less than 50 & Satisfied \\
\hline 10 & 31 & $\begin{array}{l}\text { PNE unsuccesful, } \\
\text { TLP ist stage } \\
\text { succesful }\end{array}$ & Greater than 50 & Less than 50 & Satisfied \\
\hline 11 & 18 & $\begin{array}{l}\text { PNE unsuccesful, } \\
\text { TLP ist stage } \\
\text { succesful }\end{array}$ & Greater than 50 & Less than 50 & Satisfied \\
\hline 12 & 46 & $\begin{array}{l}\text { PNE unsuccesful, } \\
\text { TLP 1st stage } \\
\text { succesful }\end{array}$ & Greater than 90 & Greater than 50 & Satisfied \\
\hline 13 & 11 & PNE succesful & Greater than 50 & Greater than 50 & Unsatisfied \\
\hline 14 & 11 & $\begin{array}{l}\text { PNE unsuccesful, } \\
\text { TLP 1st stage } \\
\text { succesful }\end{array}$ & Greater than 90 & Greater than 50 & Satisfied \\
\hline
\end{tabular}


TABLE 2 (CONTINUED)

Results of screening

$\begin{array}{lccccc}\text { Patient } & \text { Mos BoN- } & \text { Test stimula- } & \text { Leakage im- } & \text { Frequency im- } & \begin{array}{c}\text { 1-yr } \\ \text { ID }\end{array} \\ \text { TA-SNM } & \text { tion result } & \text { provement (\%) } & \text { provement (\%) } & \text { satisfaction }\end{array}$

Unsuccessful test stimulation with definitive implant not performed:

\begin{tabular}{|c|c|c|c|c|}
\hline 15 & 29 & $\begin{array}{l}\text { PNE unsuccesful, } \\
\text { TLP ist stage } \\
\text { unsuccesful }\end{array}$ & Less than 50 & Less than $5^{\circ}$ \\
\hline 16 & 18 & $\begin{array}{l}\text { PNE unsuccesful, } \\
\text { TLP 1st stage } \\
\text { unsuccesful }\end{array}$ & Less than 50 & Less than $5^{\circ}$ \\
\hline 17 & 53 & $\begin{array}{l}\text { PNE unsuccesful, } \\
\text { TLP 1st stage } \\
\text { unsuccesful }\end{array}$ & Less than 50 & Less than $5^{\circ}$ \\
\hline 18 & 18 & $\begin{array}{l}\text { PNE unsuccesful, } \\
\text { TLP 1st stage } \\
\text { unsuccesful }\end{array}$ & Less than 50 & Less than $5^{\circ}$ \\
\hline 19 & 31 & $\begin{array}{l}\text { PNE unsuccesful, } \\
\text { TLP ist stage } \\
\text { unsuccesful }\end{array}$ & Less than 50 & Less than $5^{\circ}$ \\
\hline 20 & 27 & PNE unsuccesful & Less than 50 & Less than 50 \\
\hline
\end{tabular}




\section{5 \\ DISCUSSION}

This study is the first to describe the success of SNM (test stimulation) in OAB patients, after discontinuation of BoNTA treatment. The success rate of the SNM test stimulation in our preliminary observational study was $70 \%$ (14 of 20). This finding is comparable to the published SNM test stimulation results in patients with OAB that were not previously treated with BoNTA injections.6,7 The superiority of the TLP over the PNE has been studied before, up to $22 \%$ gain in test success has been shown. 8 The 1-year satisfaction rate in this study was $79 \%$. This is comparable to the $85 \%$ satisfaction rate in patients who were treated with SNM after failure of conservative treatment. ${ }^{9}$

BoNTA and SNM are both implemented in the urological guidelines for urinary incontinence.$^{10}$ Nevertheless, their place in the treatment algorithm is still unclear and no advice is given on what treatment to choose. Predictive factors for success and efficacy of these 2 treatments have been studied extensively. Previous treatment with BoNTA has never been identified as a predictive factor for SNM success. The fact that the success rate of SNM in this study is comparable to studies indicates that in patients with idiopathic $\mathrm{OAB}$ and who have been previously treated with BoNTA, SNM can be equally successful. This finding gives clinical support to the described results in histological studies, evaluating the structural (long-term) effects of BoNTA on the urinary bladder. These studies have shown that BoNTA does not produce changes in the human urothelium or suburothelium after single or repeated injections. ${ }^{11}$ Another argument for the success of SNM after BoNTA is the difference of the working mechanism. Injection of BoNTA causes chemical denervation and reduction of cell activity, which leads to temporary inhibition of neuromuscular nerve signaling. SNM is supposed to have a modulating effect on the afferent signaling important for the coordination of storage and voiding, thereby having a central effect, where the working mechanism of BoNTA is local.

Although the lack of structural changes of the bladder after BoNTA injections, functional changes (the occurrence of chronic PVR) do occur after the in- 
jections. One of the major reasons for patients to stop BoNTA treatment is PVR, for which CISC is needed. In our study population we have included 3 patients who were still performing CISC at the time of test stimulation, with a minimum of 17 months after the last BoNTA injections. Studies have been conducted to evaluate the dose-effect ratio in relation to risk of PVR, even the use low doses (100 to $150 \mathrm{U}$ ) does not prevent the risk of PVR., ${ }^{1,2}$ Studies comparing urodynamic results before and after BoNTA treatment have showed a PVR incidence of $3 \%$ to $25 \%$ after BoNTA treatment, depending on the dose. ${ }^{12}$ In our study 2 of the 3 patients with complaints of OAB who were performing CISC since BoNTA treatment were treated successfully with SNM for their voiding and OAB complaints. This finding might indicate that SNM can have a positive effect on PVR as a consequence of BoNTA treatment.

The duration of the effect of BoNTA is not well-defined, different studies have indicated the effect to last for 6 to 12 months. ${ }^{1,13}$ The duration is suggested to be dose dependent. In our results the duration of follow-up varied between 7 and 53 months with a mean of 23 months. Remarkably all patients in whom the interval had been shorter than 12 months ( 5 of 20) experienced a successful SNM test stimulation. All these patients were dissatisfied with BoNTA, because of the lack of effect. This finding might suggest that there is no need to wait for up to 12 months before starting SNM screening, after unsatisfactory BoNTA injections.

One advantage of SNM over BoNTA injections is the long and permanent treatment effect, where BoNTA needs repeat sessions more or less annually. Long-term studies on SNM show an efficacy of 60 to $70 \%$ at a mean followup of 26 to 64 months..$^{14-16}$

One year after the definitive implant $79 \%$ (11 of 14) of the patients indicated to be satisfied with the effect of SNM treatment. Unfortunately we have only evaluated the subjective patient satisfaction, because no validated questionnaires had been filled out. The recurrence of incontinence, which could not be controlled with changes in programming settings, was the reason for dissatisfaction of 3 patients. There were no signs of lead migration or other technical failure of the system. Of these 3 patients 1 in whom SNM failed had a history of psychiatric disease, which is known to be a predictive factor for encountering adverse events (i.e. loss of efficacy) in permanent SNM treatment. ${ }^{17}$

The small sample size is a limitation of this study. Although the BoNTA treatment has emerged to a widely performed treatment and has a $37 \%$ discontinuation rate, the sample size of our recruitment shows little patients are referred to a tertiary referral center. 
Despite the small sample size this first observational study demonstrates the possible role of SNM after BoNTA treatment. However, it does not give final guidance on the place of both interventions in the treatment algorithm. SNM and BoNTA are both regarded as minimally invasive, well accepted and safe. The success rates of the treatments (both up to $70 \%$ ) are difficult to compare, because both treatments are evaluated differently. ${ }^{1,14,18,19}$

Studies on BoNTA are analyzed by intent to treat, were SNM is analyzed on the per protocol principle. Besides comparable clinical effectiveness, a recent study indicates that SNM compared to BoNTA for patients with idiopathic $\mathrm{OAB}$ is cost-effective during a period of 5 years. ${ }^{20}$ In our opinion, for the time being, it is up to the physician in consultation with the patient to decide which treatment to start with after failure of conservative treatments, considering all pros and cons. 


\section{6 \\ CONCLUSIONS}

Patients who are dissatisfied with or in whom BoNTA fails can respond successfully to sacral neuromodulation. The response rate of the test stimulation and the satisfaction at 1-year follow up is comparable to the response rate of patients who have received SNM immediately after conservative treatment. The place of both treatments in the treatment algorithm of OAB needs to be defined by future studies. 


\section{7 \\ REFERENCES}

1 Denys P, Le Normand L, Ghout I et al: Efficacy and safety of low doses of botulinum toxin type a for the treatment of refractory idiopathic overactive bladder: a multicentre, double-blind, randomised, placebo-controlled dose-ranging study. Eur Urol 2012; 61: 520.

2 Dmochowski R, Chapple C, Nitti VW et al: Efficacy and safety of onabotulinumtoxinA for idiopathic overactive bladder: a double-blind, placebo controlled, randomized, dose ranging trial. J Urol 2010; 184: 2416.

3 Sahai A, Khan MS and Dasgupta P: Efficacy of botulinum toxin-A for treating idiopathic detrusor overactivity: results from a single center, randomized, double-blind, placebo controlled trial. J Urol 2007; 177: 2231.

4 Brubaker L, Richter HE, Visco A et al: Refractory idiopathic urge urinary incontinence and botulinum A injection. J Urol 2008; 180: 217.

5 Dowson C, Watkins J, Khan MS et al: Repeated botulinum toxin type A injections for refractory overactive bladder: medium-term outcomes, safety profile, and discontinuation rates. Eur Urol 2012; 61: 834.

6 Marcelissen T, Leong R, Serroyen J et al: Is the screening method of sacral neuromodulation a prognostic factor for long-term success? J Urol 2011; 185: 583.

7 Al-zahrani AA, Elzayat EA and Gajewski JB: Long-term outcome and surgical interventions after sacral neuromodulation implant for lower urinary tract symptoms: 14-year experience at 1 center. J Urol 2011; 185: 981.

8 Leong RK, De Wachter SG, Nieman FH et al: PNE versus 1st stage tined lead procedure: a direct comparison to select the most sensitive test method to identify patients suitable for sacral neuromodulation therapy. Neurourol Urodyn 2011; 30: 1249.

9 Leong RK, Marcelissen TA, Nieman FH et al: Satisfaction and patient experience with sacral neuromodulation: results of a single center sample survey. J Urol 2011; 185: 588.

10 Gormley EA, Lightner DJ, Burgio KL et al: Diagnosis and treatment of overactive bladder (non-neurogenic) in adults: AUA/SUFU Guideline. American Urological Association Education and Research, Inc 2012; p 36.

11 Apostolidis A, Jacques TS, Freeman A et al: Histological changes in the urothelium and suburothelium of human overactive bladder following intradetrusor injections of botulinum neurotoxin type A for the treatment of neurogenic or idiopathic detrusor overactivity. Eur Urol 2008; 53: 1245 .

12 Rovner E, Kennelly M, Schulte-Baukloh H et al: Urodynamic results and clinical outcomes with intradetrusor injections of onabotulinumtoxinA in a randomized, placebo-controlled dose-finding study in idiopathic overactive bladder. Neurourol Urodyn 2011; 30: 556.

13 Kuo $\mathrm{HC}$, Liao $\mathrm{CH}$ and Chung SD: Adverse events of intravesical botulinum toxin a injections for idiopathic detrusor overactivity: risk factors and influence on treatment outcome. Eur Urol 2010; 58: 919. 
14 Marcelissen TA, Leong RK, de Bie RA et al: Long-term results of sacral neuromodulation with the tined lead procedure. J Urol 2010; 184: 1997.

15 Siegel SW, Catanzaro F, Dijkema HE et al: Long-term results of a multicenter study on sacral nerve stimulation for treatment of urinary urge incontinence, urgency-frequency, and retention. Urology 2000; 56: 87.

16 van Voskuilen AC, Oerlemans DJ, Weil EH et al: Long term results of neuromodulation by sacral nerve stimulation for lower urinary tract symptoms: a retrospective single center study. Eur Urol 2006; 49: 366.

17 Marcelissen TA, Leong RK, Nieman FH et al: Psychological and psychiatric factors as predictors for success in sacral neuromodulation treatment. BJU Int 2011; 108: 1834.

18 Anger JT, Weinberg A, Suttorp MJ et al: Outcomes of intravesical botulinum toxin for idiopathic overactive bladder symptoms: a systematic review of the literature. J Urol 2010; 183: 2258.

19 Chartier-Kastler E: Sacral neuromodulation for treating the symptoms of overactive bladder syndrome and non-obstructive urinary retention: >10 years of clinical experience. BJU Int 2008; 101: 417.

20 Leong RK, de Wachter SG, Joore MA et al: Cost-effectiveness analysis of sacral neuromodulation and botulinum toxin A treatment for patients with idiopathic overactive bladder. BJU Int 2011; 108: 558 . 
CHAPTER 7 


\section{Sacral Neuromodulation in Patients with Combined Micturition and Defecation Disorders}

Dennis J.A.J Oerlemans, Tom A.T. Marcelissen, Jamie M. Drossaerts, Jarno Melenhorst, Philip E.V.A. Van Kerrebroeck

Submitted for publication 


\section{7-1}

\section{ABSTRACT}

\section{Introduction}

Functional disorders of the lower urinary tract often coexist with defecation disorders and sexual disorders. Although evacuation and storage disorders are often seen as separate entities, combined symptoms of urinary storage and bowel evacuation (or vice versa) also occur.

\section{Materials and Methods}

In this single center observational study, we included all patients with combined micturition and defecation disorders who were treated with SNM in our institute between 2008 and 2013. The primary outcome measure was improvement in leakage, defined as a greater than $50 \%$ improvement in episodes of urine leakage and severity of leakage or an improvement in the fecal incontinence or constipation episodes. At the routine annual outpatient check-up, the patients who received the definitive SNM implant were asked about their satisfaction.

\section{Results}

In total 105 patients were included and were tested for SNM treatment in the period between 1997 and 2014. Of all the patients tested, 59 (56.2\%) were implanted with a definitive system. In 39 patients there was a successful result for only urinary symptoms. In 15 patients there was a successful result for both symptoms, and in 2 patients only for their fecal symptoms.

\section{Conclusions}

SNM is a feasible treatment option for patients with combined micturition and defecation disorders 


\section{$7 \cdot 2$}

\section{INTRODUCTION}

Functional disorders of the lower urinary tract often coexist with defecation disorders and sexual disorders. Anatomically, the bladder, anorectum and reproductive organs are closely related and all depend on the integrity and coordination of the common innervation of the pelvic floor muscles. The etiology of pelvic floor disorders is complex and multifactorial. Different factors can be involved, such as neural (e.g. pudendal nerve injury), mechanical (e.g. pelvic organ prolapse) or systemic (e.g. MS or Parkinson's disease). Depending on the exact changes in these factors, both evacuation disorders (urinary retention, obstipation) as well as storage disorders (urinary and fecal incontinence) can occur. Although evacuation and storage disorders are often seen as separate entities, combined symptoms of urinary storage and bowel evacuation (or vice versa) also occur.

In our institution, an academic teaching hospital, patients with pelvic floor disorders are treated in a multidisciplinary team, including a urologist, surgeon, gynecologist and physiotherapist. Almost $74 \%$ of our patients report voiding dysfunctions and more than $23 \%$ of our patients report bowel dysfunction. ${ }^{1}$ When conservative treatment fails, patients with combined problems can be treated with Sacral Neuromodulation (SNM). Sacral neuromodulation works by direct stimulation of the nerve roots in the sacral S3 foramen via an implanted electrode. The effect can be attributed to the modulation of afferent nerve signals in the spinal cord. SNM was first applied in patients with urinary incontinence. Later, this treatment proved to be useful in treating other pelvic floor disorders, including urinary retention, fecal incontinence and obstipation. ${ }^{2}$ Since the innervation of the pelvic floor organs is mediated by the same pathways, it has been postulated that SNM restores normal neural activity in the affected organ (e.g. overactive bladder, sphincter hypertonicity, or hypocontractility).

In this study, we evaluated the clinical results of sacral neuromodulation in patients with combined urinary and fecal symptoms. Furthermore, a review of the literature on the treatment of combined problems with SNM was performed. 


\section{3}

\section{MATERIAL AND METHODS}

In this single center observational study, we included all patients with combined micturition and defecation disorders who were treated with SNM in our institute between 2008 and 2013. All patients were primarily treated for their micturition symptoms. The symptoms consisted of overactive bladder syndrome (with or without urgency incontinence) or non-obstructive urinary retention. The concurrent defecation disorder symptoms included fecal incontinence or constipation. Fecal incontinence was defined as involuntary loss of stool at least once a week, which was documented by means of a 3-week bowel diary.

History taking and a 3 -day voiding diary assessed the urological complaints. The voiding diary as well as a defecation diary were used as a baseline. Physical examination and urinalysis were used to rule out other conditions. Urodynamics were performed in all patients to confirm the diagnosis of detrusor overactivity and to exclude other diagnoses.

Physiological assessment included anal manometry using a Konigsberg catheter (Konigsberg Instrument Inc., Pasadena, California, USA) connected to a computer-assisted polygraph (Synectics Medical, Stockholm, Sweden). A history of anorectal malformation, rectal surgery within the past 12 months, presence of a rectal prolapse, rectocele or intussusception, inflammatory bowel disease and chronic diarrhea were exclusion criteria. All patients underwent SNM test stimulation with use of the PNE test or a tined lead test stimulation.

During the test stimulation period, a 3-day voiding diary and a 2-week bowel diary were again filled out to evaluate the effect of SNM. The test stimulation was evaluated by comparing the data of the diaries filled out during the test stimulation and the baseline voiding diary.

The primary outcome measure was improvement in leakage, defined as a greater than $50 \%$ improvement in episodes of urine leakage and severity of leakage or an improvement in the fecal incontinence or constipation episodes.

In the event of successful PNE, this was followed by a 1-stage TLP with definitive placement of an implantable neurostimulator (InterStim ${ }^{\circledR}$ I or InterStim II, 
Medtronic Inc). In the event of unsuccessful PNE test stimulation, a first stage TLP was performed. When the first stage was successful, the second stage of the TLP consisted of a definitive placement of an implantable pulse generator. Several pulse generator settings were used, preferably unipolar electrode selection with a frequency of $10 \mathrm{~Hz}$, a pulse width of 210 us and an amplitude just above sensation threshold (preferably lower than 2.0 V). The technical procedure for sacral neuromodulation has been described previously. ${ }^{3}$

At the routine annual outpatient check-up, the patients who received the definitive SNM implant were asked about their satisfaction. The data processing of this study was performed with IBM SPSS ${ }^{\circledR}$ statistics, version 18 . 


\section{4}

\section{RESULTS}

In total 105 patients were included and were tested for SNM treatment in the period between 1997 and 2014. Out of all the patients, 81 were female and 24 male. The primary (urinary) symptom was $\mathrm{OAB}$ (wet or dry) in 68 patients and non-obstructive urinary retention in 37 patients. The secondary (fecal) symptom was FI in 37 patients and constipation in 59 patients. Six patients reported both FI and constipation, 3 had other (urgency, anorectal pain) fecal problems. Table 1 summarizes the combination of micturition and fecal symptoms at baseline.

TABLE 1

Combinations of urinary and fecal symptoms at baseline

\begin{tabular}{lccc} 
Fecal symptoms & $\begin{array}{c}\text { Urinary symptoms: } \\
\text { Retention }\end{array}$ & $\begin{array}{c}\text { Urinary symptoms: } \\
\text { OAB }\end{array}$ & Total \\
\hline Fecal incontinence & 5 & 32 & 37 \\
\hline Constipation & 30 & 29 & 59 \\
\hline Both & 2 & 4 & 6 \\
\hline Other & 0 & 3 & 3 \\
\hline Total & 37 & 68 & 105 \\
\hline
\end{tabular}


Forty-three patients were first tested with a PNE procedure, and the test stimulation was successful in 28 (65.2\%) patients. Ninety-one TLP procedures were performed, 77 were 2-stage procedures and 14 single-stage procedures. Of all the patients tested, 59 (56.2\%) were implanted with a definitive system. In 39 patients there was a successful result for only urinary symptoms. In 15 patients there was a successful result for both symptoms, and in 2 patients only for their fecal symptoms. Table 2 shows the results of all patients tested with TLP, stratified by the combination of symptoms.

TABLE 2

Success rate of TLP test stimulation, stratified by combination of urinary and fecal symptoms

\begin{tabular}{lccc}
$\begin{array}{l}\text { Combination of } \\
\text { symptoms }\end{array}$ & $\begin{array}{c}\text { TLP succes: } \\
\text { Yes }\end{array}$ & $\begin{array}{c}\text { TLP succes: } \\
\text { No }\end{array}$ & \% Succes \\
\hline UR + FI & 1 & 2 & $33 \%$ \\
\hline UR + constipation & 11 & 13 & $46 \%$ \\
\hline UR + both & 1 & 1 & $50 \%$ \\
\hline OAB + FI & 20 & 9 & $68 \%$ \\
\hline OAB + constipation & 19 & 7 & $73 \%$ \\
\hline OAB + both & 4 & 0 & $100 \%$ \\
\hline OAB + other & 3 & 0 & $100 \%$ \\
\hline Total & 59 & 32 & \\
\hline
\end{tabular}




\section{5}

\section{DISCUSSION}

The lower urinary tract, anorectal channel and pelvic floor are closely related, both anatomically and functionally. Dysfunction of the lower urinary tract and dysfunction of the bowel are problems that can have a major impact on the quality of life. Patients may complain about multiple symptoms: urgency and frequency, urgency urinary incontinence, urinary retention, genito-urinary pain syndromes and also fecal incontinence and constipation. Fecal incontinence (FI) is a common symptom that has a devastating impact on the quality of life. Reported prevalence ranges from 7-15\% in community dwelling men and women. ${ }^{4}$ Because healthcare providers do not often screen for these symptoms and patients do not voluntarily report them, the real prevalence is probably underreported. Constipation presumably has less impact on the quality of daily life, however it represents a significant burden and is often associated with FI or urinary problems. SNM is a well-established therapy for both urinary and fecal complaints. ${ }^{5,6}$ For both indications, the working mechanism of SNM is not completely known.? SNM probably involves different modes of action and acts on various levels of the nervous system, including sacral, spinal, supraspinal and cortical areas.

The various urinary and fecal disorders all demand a specific treatment approach, and sometimes therapies can have bothersome interactions. For example, OAB can be treated with anticholinergic drugs, however, these drugs can have a negative impact on constipation. Surgery of the anorectum or pelvic organ prolapse can increase urinary urgency. Hence, treatment of patients with combined symptoms can be a challenge. SNM has the advantage that both micturition and defecation can improve with the same therapy. SNM has several advantages compared to other available techniques.

Compared to percutaneous tibial nerve stimulation (PTNS), SNM has the advantage of the duration of its effect. Furthermore PTNS has to be repeated weekly. The efficacy for PTNS in patients with fecal complaints could not be proven with the CONFIDeNT trial, a double-blind, multi centre, pragmatic, par- 
allel-group, randomized controlled trial. The authors concluded that PTNS given for a period of 12 weeks did not confer significant clinical benefit over sham electrical stimulation in the treatment of adults with fecal incontinence. ${ }^{8}$

Long-term results with SNM therapy differ between the indications. After five years of follow-up, positive results were seen in $56 \%$ of patients with $O A B$ symptoms compared to $71 \%$ in patients with urinary retention. No life-threatening or irreversible adverse events occurred during this long-term follow-up. ${ }^{5}$ Long-term results with SNM for fecal incontinence and constipation range from 54 to $63 \% .^{9}$ Although the course of treatment for patients with combined symptoms was mentioned in earlier studies ${ }^{10}$, to our knowledge only one study reported long-term results for these patients. ${ }^{11}$ El-Gazzaz et al reported on SNM in 24 patients with combined symptoms, of whom 10 showed a significant improvement at a mean follow-up of 24 months. Four patients in this study had their system explanted; in 2 patients this was due to a lack of clinical result for one of the symptoms, and in 2 other patients due to infection.

We describe a population of 105 patients, both men and women. In our study, $25 \%$ of implanted patients showed $>50 \%$ improvement in both urinary and fecal symptoms. Although this rate might seem low, these patients have a clinically significant reduction in both symptoms, most probably leading to a high overall satisfaction and increase in quality of life. As far as we know, there is no other single-modality treatment that can have this impact on patients with combined urinary and fecal symptoms. We found the highest response to SNM in patients with $\mathrm{OAB}$ as the primary complaint (see table 2). Both the combination of FI and constipation showed a success rate of approximately $70 \%$. The reason for patients with urinary retention responding less to SNM could be that patients with both UR and constipation represent a specific patient group with a specific pathophysiology (e.g. bladder/bowel underactivity) that is less suitable for neuromodulation.

Although long-term results are lacking, the effect of SNM in patients with isolated urinary or fecal symptoms appears to be durable, with reported efficacy rates of $70 \%$ after 5 years. Caremel et al conducted a survey among patients with double incontinence. They interviewed 37 patients who were implanted with a neurostimulator (on average 30 months since implantation) about their urinary and fecal complaints. In total, $49 \%$ reported an improvement in both complaints. ${ }^{12}$ Predictors for SNM success have been studied previously but no clear factor is repeatedly reported. ${ }^{13-15}$

SNM has been proven to be a safe therapy. All patients tested in this study who experienced negative results were explanted without complications. 
In the group of implanted patients, 13 re-operations were performed due to complications; in total 5 revisions of the lead, 5 revisions of the pocket where the IPG was implanted and 3 re-operations due to infections. 


\section{6 \\ CONCLUSIONS}

With this study we have shown that SNM is a feasible treatment option for patients with combined micturition and defecation disorders. In $25 \%$ of all patients, more than a 50\% improvement in both urinary and fecal symptoms can be accomplished. As there may be a common etiology, SNM may work for both urinary and fecal problems. Unfortunately, in the majority of patients the etiology is unknown. Patients with combined symptoms who experience symptoms of OAB might be the better candidates for SNM than those with a combination with urinary retention. 


\section{7 \\ REFERENCES}

1 Berghmans B, Nieman F, Leue C, Weemhoff M, Breukink S, van Koeveringe G. Prevalence and triage of first contact pelvic floor dysfunction complaints in male patients referred to a Pelvic Care Centre. Neurourology and urodynamics 2015.

2 Matzel K, Stadelmaier U, Hohenfellner M, Gall F. Electrical stimulation of sacral spinal nerves for treatment of faecal incontinence. Lancet 1995 346:1124-7.

3 Oerlemans DJ, van Kerrebroeck PE. Sacral nerve stimulation for neuromodulation of the lower urinary tract. Neurourology and urodynamics 2008;27:28-33.

4 Bharucha AE, Dunivan G, Goode PS, et al. Epidemiology, Pathophysiology, and Classification of Fecal Incontinence: State of the Science Summary for the National Institute of Diabetes and Digestive and Kidney Diseases (NIDDK) Workshop. The American journal of gastroenterology 2015;110:127-36.

5 van Kerrebroeck PE, van Voskuilen AC, Heesakkers JP, et al. Results of sacral neuromodulation therapy for urinary voiding dysfunction: outcomes of a prospective, worldwide clinical study. The Journal of urology 2007;178:2029-34.

6 Thin NN, Horrocks EJ, Hotouras A, et al. Systematic review of the clinical effectiveness of neuromodulation in the treatment of faecal incontinence. The British journal of surgery 2013;100:1430-47.

7 Amend B, Matzel K, Abrams P, de Groat W, Sievert K. How does neuromodulation work. Neurourology and urodynamics 2011;30:762-5.

8 Knowles $\mathrm{CH}$, Horrocks EJ, Bremner SA, et al. Percutaneous tibial nerve stimulation versus sham electrical stimulation for the treatment of faecal incontinence in adults (CONFIDeNT): a double-blind, multicentre, pragmatic, parallel-group, randomised controlled trial. Lancet 2015 .

9 Thin NN, Horrocks EJ, Hotouras A, et al. Systematic review of the clinical effectiveness of neuromodulation in the treatment of faecal incontinence. British Journal of Surgery 2013;100:1430-47.

10 Uludag O, Melenhorst J, Koch SM, van Gemert WG, Dejong CH, Baeten CG. Sacral neuromodulation: long-term outcome and quality of life in patients with faecal incontinence. Colorectal disease : the official journal of the Association of Coloproctology of Great Britain and Ireland 2011;13:1162-6.

11 El-Gazzaz G, Zutshi M, Salcedo L, Hammel J, Rackley R, Hull T. Sacral neuromodulation for the treatment of fecal incontinence and urinary incontinence in female patients: long-term follow-up. International journal of colorectal disease 2009;24:1377-81.

12 Caremel R, Damon H, Ruffion A, et al. Can sacral neuromodulation improve minor incontinence symptoms in doubly incontinent patients successfully treated for major incontinence symptoms? Urology 2012;79:80-5. 
13 Drossaerts J, Rademakers K, van Koeveringe G, Van Kerrebroeck P. The value of urodynamic tools to guide patient selection in sacral neuromodulation. World journal of urology 2015.

14 Marcelissen TA, Leong RK, Nieman FH, van Lankveld JJ, van Kerrebroeck PE, de Wachter SG. Psychological and psychiatric factors as predictors for success in sacral neuromodulation treatment. BJU international 2011;108:1834-8.

15 Duelund-Jakobsen J, van Wunnik B, Buntzen S, Lundby L, Laurberg S, Baeten C. Baseline factors predictive of patient satisfaction with sacral neuromodulation for idiopathic fecal incontinence. International journal of colorectal disease 2014;29:793-8. 
CHAPTER 8 
General Discussion 


\section{8-1}

\section{INTRODUCTION}

Sacral Neuromodulation (SNM) is an established treatment for patients with refractory lower urinary tract dysfunction. Since the FDA approval in 1997 there have been many advancements in technical and surgical aspects which have improved treatment safety and efficacy. These include the percutaneous tined lead, use of local anesthesia and fluoroscopy, and a smaller implantable stimulator. This thesis gives an overview of the clinical experience with SNM treatment. Also, clinical experience and continuous research resulted in a better understanding of the application of SNM. This has improved patient selection, the application of this technique and the management of complications during follow-up. Besides SNM, other neuromodulatory techniques have been developed over the last decades, including transcutaneous electrical nerve stimulation, percutaneous tibial nerve stimulation and pudendal nerve stimulation. Together with the use of intravesical botulinum toxin type-A injections, these treatments have completely changed the specialized management of the overactive bladder syndrome. In this section we will discuss the evolution of SNM treatment. We will present an overview of the most important improvements in the past, the role of SNM compared to other third-line treatments at present, and the role of SNM and opportunities for improvement in the future. 


\section{2 \\ PAST}

In the 1980's Tanagho et al. developed a clinically applicable method for SNM by using an implantable stimulator. ${ }^{1}$ At first, the implantation of the electrode was regarded as a complex and time-consuming procedure. The stimulation lead was positioned in the sacral foramen under direct vision using a large midline incision. After correct placement, the lead was secured to the sacral periosteum. Although treatment results of this early technique were good, complications such as wound infection and post-operative pain were relatively frequent. Moreover, there was uncertainty whether the positive effects of SNM were durable. As outlined in Chapter 3, we analysed the long-term results of SNM in a large group of patients that were treated in our center using the initial technique. We showed that SNM is a safe and durable therapy for patients with refractory symptoms of $\mathrm{OAB}$ and non-obstructive urinary retention. In addition, the number of revision rates due to complications steadily decreased with the growing experience in our center. Patients implanted before 1995 had a considerably higher rate of adverse events compared to patients implanted after 1995. An important reason for this difference is the improvement of patient selection and technical aspects of the implantation procedure. In 1994 a new pulse generator (Itrell-II) and electrode became available. The same phenomenon was seen in 1999 when an updated pulse generator, the Interstim I device and buttock placement of the pulse generator were introduced. We can conclude that technical developments markedly improved SNM therapy over the years.

In 2002, a percutaneous technique for implantation of the stimulation lead was introduced, also called the tined lead procedure (TLP), this replaced the open procedure. Spinelli et al were the first to publish on the use of this less invasive self-anchoring lead. ${ }^{2}$ The tined lead can be placed under local anesthesia and sensory information during intra-operative stimulation can be used to search for the perfect location for stimulation. The quadripolar tined lead consists of four sets of tines proximal to the electrodes, which engage the subcutaneous tissue and muscle around the lead to prevent migration ${ }^{2}$. The tined lead 
made it possible to perform a longer test phase (2-3 weeks) with the definitive lead implanted. If this test period showed positive results the pulse generator could be implanted during a second stage of the surgical procedure. Another major advantage of the TLP procedure is that patients can experience in real life how it feels to have constant stimulation. Our results with the tined lead technique are described in chapter 4 . We concluded that this new minimally invasive approach for SNM is easier to perform than the classic open method. It gives positive results in both the short- and medium-term. Two-stage testing with the tined lead appears to be a more reliable method than the classic PNE because of a prolonged testing period and perhaps because of the lower risk of electrode migration. Therefore testing with a tined lead is a useful alternative for patients with an inconclusive PNE result. Stimulation is given by a permanent lead with four contact points and can be unipolar as well as bipolar. As there was only one lead migration during the medium-term follow-up of up to 3 years, it is obvious that the lead anchoring method is sufficient for fixing the electrode. A follow-up study of the patients in the study mentioned above shows that the tined lead is also effective and safe in the long-term, with a success rate of approximately $70 \%{ }^{3}$ The incidence of lead migration was rather low (1.6\%), indicating that the self-anchoring technique is reliable. Due to these convincing results, the TLP has replaced the classic open technique. The minimally invasive nature of the TLP also makes it ideal for test stimulation. Compared with PNE, a longer trial stimulation can be conducted with superior lead fixation, making the test stimulation more reliable. ${ }^{4-6}$ Although TLP is a better tool for selecting eligible candidates for SNM than PNE, the long-term outcome for patients screened with either of these tools is comparable.? Hence, both these tools can still be used in daily practice, depending on the specific indication and physician or patient preference.

The original IPG (Interstim I) has an average battery life of 7-10 years. It is preferred in patients who require high voltage for stimulation in order to maximize the time until IPG replacement. The InterStim II device, introduced in 2006, is 50\% lighter and smaller. The smaller generator allows for a smaller incision and pocket to be created leading to less discomfort and higher patient acceptance. ${ }^{4}$ It is ideal in lean patients, pediatric patients and those with lower stimulation intensity requirements determined at the time of test stimulation. However due to the smaller size, the average battery life is only three to five years. 


\section{Predictive Factors}

Many papers reported on predictive factors for a successful result with SNM. Scheepens et al. already tested 212 patients with a PNE test in 2002. They found that intervertebral disk prolapse, duration of complaints, neurogenic bladder dysfunction, and urgency incontinence were found to be significant predictive factors. ${ }^{8}$

Presumably, the incidence and degree of psychological problems might have an effect on the treatment outcome of SNM, and psychiatric comorbidity has previously been proposed as a negative predictor by some authors. However, those results were based on small patient groups, with retrospective data. The role of a broad spectrum of psychological factors was prospectively evaluated in relation to SNM treatment by using validated psychological screening questionnaires. The relationship between psychiatric history and the outcome of chronic SNM treatment was also assessed. The conclusion was that there was no evidence that psychological screening with the ABQ or SCL-90-R can predict the outcome of SNM treatment. Patients with a medical history of psychiatric disease appear to be more likely to encounter adverse events with permanent SNM treatment. ${ }^{9}$ These results were confirmed in a more recent paper which concluded that although it is known that psychological factors play a role in the severity of LUTS, they do not predict SNM outcome. ${ }^{10}$

With the introduction of the tined lead placement, the possibility to implant a definitive system under local anesthesia was created. An advantage could be that the sensory response could give direct feedback during lead placement. It was hypothesized that this could give better results. Peters et al tested 141 subjects (82\% female), of which 86 (61\%) had sensory testing and 55 (39\%) did not. They had to conclude that intraoperative sensory testing during sacral lead placement does not necessarily improve IPG implantation rates or clinical outcomes of SNM." Although sensory testing in this large group seemed not to improve the results, in individual cases the sensory feedback can be very useful. Further research will hopefully give more direction in selecting good candidates for SNM.

\section{Urodynamics}

Different groups found a correlation between SNM results and changes in urodynamic studies. In 1997 Everaert et al. published their results of urodynamic evaluation of SNM in patients with voiding dysfunction. ${ }^{12}$ They concluded that ideal candidates for SNM are those patients with a spastic pelvic floor syndrome or with a hypo-contractile detrusor, in combination with sphincter instabili- 
ty, and impaired sphincter relaxation. An increase of bladder contractility, enhancement of sphincter relaxation and decrease in bladder capacity and residual urine were the most important features of the response in the patients they studied.

An analysis of ambulatory urodynamic data in patients with an overactive bladder was presented in 2003. Thirtyfour patients were studied, for 22 patients a detrusor activity index (DAI) could be calculated. A significant correlation ( $\mathrm{p}$ o.03) was found in DAI reduction of the ambulant urodynamic studies before and during SNM as compared to clinical improvement in overactive bladder symptoms. ${ }^{13}$

Other groups found a significant correlation between treatment efficacy and urodynamic changes in $\mathrm{OAB}$ patients and in patients with urinary retention treated with SNM. ${ }^{14-17}$

Very recently the value of urodynamic tools to guide patient selection in SNM were studied..$^{18}$ With this study the authors explored whether urodynamic testing with the addition of ambulatory urodynamic study (UDS), is able to better predict and assess SNM treatment outcome. 98 patients with lower urinary tract symptoms (storage and/or voiding dysfunction) were included in an ambulatory urodynamic measurement database. The success rate of SNM in patients with storage dysfunction was around $70 \%$, according to either conventional-UDS or ambulatory-UDS diagnosis. Based on conventional-UDS, the success rate of SNM in patients with hypocontractility was $67 \%$ and in acontractile patients $35 \%$. According to ambulatory-UDS diagnosis, success rates were 32 and $17 \%$, respectively. This study showed that conventional-UDS overestimates the amount of patients diagnosed with a hypocontractile or acontractile bladder. Patients with reduced contractility on ambulatory-UDS were shown to have a lower chance of SNM success. Hence, ambulatory-UDS facilitated the selection of patients with a real a-contractile bladder and predicted SNM failure. In patients with storage dysfunction, additional ambulatory-UDS did not seem to contribute in predicting SNM outcome.

\section{Bilateral Stimulation}

Bilateral SNM has been proposed as a more effective treatment for chronic voiding dysfunction; a prospective randomized study with a relatively small group of 33 patients tested with a PNE test showed that bilateral is generally not superior to unilateral SNM. ${ }^{19}$ However, in some individuals bilateral stimulation has been shown to be more effective in relieving symptoms. Therefore the authors concluded that if unilateral stimulation fails, a bilateral test should be consid- 
ered. Even more interesting was a study with patients who had all failed unilateral stimulation. In this small group 4 out of 12 analyzed patients had a positive response to bilateral stimulation after they had failed unilateral stimulation2o. Further investigation will be necessary to determine the real value of bilateral stimulation.

\section{Stimulation Parameters}

In most individuals treated with SNM for LUTD standard stimulation parameters can be used. Some patients however did have a loss or reduction of effect over time in which case the stimulation parameters could be changed to restore adequate stimulation.

Some studies were performed to search for optimal stimulation parameters. In a study with 50 patients looking for optimal parameters four different sets of parameters were tested; 41 of the patients included $(82 \%)$ had overactive bladder symptoms and $9(18 \%)$ had chronic non-obstructive urinary retention. No significant difference was found in the clinical outcome of the voiding diary and questionnaire between the pulse rates and none of the 4 rates was significantly related to SNM associated pain. However, individuals appeared to benefit from changing the pulse rate in terms of treatment efficacy and stimulation related pain. ${ }^{21}$

Another study searching for the ideal stimulation rates used a $3 \times 3$ orthogonal Latin squares crossover design to assess variability in overactive bladder symptoms and adverse events when subjects were exposed to three rate settings of SNM. Twelve subjects completed the study. Upon enrollment, each subject was randomized to one of three rate-setting sequences: 5.2, 14, and $25 \mathrm{~Hz}$. Each subject tested each rate setting for 1 week. The rate significantly affected the number of incontinence episodes and pad changes per day. The number of adverse events was similar across the three rate settings with programming-related adverse events lowest in the $14 \mathrm{~Hz}$ group. ${ }^{22}$

In the treatment of fecal constipation, a small double-blind randomized multicenter study was performed. ${ }^{23}$ This study aimed to see if alteration of the pulse width or frequency improved the outcome for those with constipation. Eleven patients with constipation being treated by SNM were recruited from three centers. They were randomized to five different stimulation protocols each of which was applied for 5 weeks. Group 1 used standard settings (pulse width $210 \mu \mathrm{s}$, frequency $14 \mathrm{~Hz}$ ); in the other four groups (Groups 2-5) the pulse width and/or frequency were halved or doubled. Patients and investigators were unaware of the group allocation. The Cleveland Clinic constipation score varied 
significantly between the five groups. Group 1 achieved the lowest mean score $( \pm$ SD) $13.4( \pm 4.4)(\mathrm{P}=0.03)$. The number of digitations per defecation was the lowest in Group 4, $90 \mu$ s and $14 \mathrm{~Hz}(\mathrm{P}<0.01)$. No other variable changed significantly. The recruited patients generally preferred the standard settings. 


\section{8-3 \\ PRESENT}

The position of SNM as a third-line treatment in the treatment algorithm for OAB has not been proven yet. Various guidelines recommend the use of SNM, PTNS or BoNT-A, without mentioning a preferred sequence. Also, with the introduction of mirabegron, a new pharmacological option has arisen for the treatment of refractory OAB patients. The choice for a third-line therapy is dependent on multiple factors including patient preference, surgical expertise, available resources and finances. Hence the current treatment evidence and possible considerations in the third-line management of OAB remains an important point of discussion.

\section{Pharmacologic Therapy}

Various medications with antimuscarinic effects are available for the treatment of OAB symptoms. The most commonly used are oxybutinin, tolterodine, solifenacin, darifenacin, fesosterodine and trospium. Several studies have reviewed the efficacy and adverse events of these drugs. Although all drugs have similar efficacy, oxybutynin was associated with more side effects compared to the others. ${ }^{24-26}$ Antimuscarinics provide a health-related quality of life benefit to patients with $\mathrm{OAB}$, with no significant difference between various agents. ${ }^{27}$

Mirabegron is a selective b3-adrenoreceptor agonist and has a different pharmacological profile and mechanism of action than antimuscarinics. Bladder relaxation is obtained by the activation of the b3-adrenoceceptor and the subsequent activation of adenylyl cyclase, which catalyses the conversion of adenosine triphosphate (ATP) into cyclic adenosine monophosphate (cAMP). Six large randomized trials have evaluated the efficacy and adverse events of mirabegron compared to placebo (or an active control group) and showed that mirabegron is efficacious and safe in treating patients with OAB. ${ }^{28-30}$ The clinical results were in line with those observed with well-established antimuscarinic treatments. However, there is a lack of evidence for long-term efficacy yet. While mirabegron is effective and well tolerated in the short-term, its place in the treatment 
algorithm of OAB remains debatable. Some guidelines have already adapted the use of mirabegron as a possible first-line treatment (AUA) whereas others recommend its use in patients who do not respond to antimuscarinics (NICE). The number of pharmacological agents that should be tried before commencing more invasive treatment is also arguable.

Combination therapy with antimuscarinics and mirabegron might be a promising option for refractory OAB patients. In the SYMPHONY trial, a total of 1306 patients were randomised to 12 week combination of solifenacin/ mirabegron or monotherapy with solifenacin or mirabegron, or placebo. ${ }^{31} \mathrm{Com}$ bination therapy with solifen-acin/mirabegron was well tolerated and significantly improved OAB symptoms compared with solifenacin $5 \mathrm{mg}$ monotherapy. Yamaguchi et al. evaluated the effect of additional therapy with mirabegron in patients treated with solifenacine. ${ }^{32}$ Patients who were being treated with solifenacin at a stable dose for at least 4 weeks were included. Patients continued to receive solifenacin ( 2.5 or $5 \mathrm{mg}$ once daily) and additional mirabegron ( 25 $\mathrm{mg}$ once daily) for 16 weeks. After 8 weeks of treatment, the mirabegron dose could be increased to $50 \mathrm{mg}$ if symptom improvement was not sufficient. There were significant improvements in OAB symptoms while using the combination therapy with mirabegron and solifenacin. The combination therapy was well tolerated, with the most common adverse effect being constipation.

Currently, the potential of combination treatment with mirabegron and solifenacin is being explored further by the SYNERGY trial (ClinicalTrials.gov id: NCTo1340027) and the BESIDE trial (ClinicalTrials.gov id: NCTo1908829).

\section{SNM}

The U.S. Food and Drug Administration (FDA) has approved SNM in 1997 for both urgency incontinence and urgency-frequency syndrome in refractory $\mathrm{OAB}$. There is convincing evidence for the success of SNM with positive longterm results regarding efficacy and safety. ${ }^{33,34}$ Eight randomized studies and many long-term observational studies have been published, with a reported clinical response rate between 64 and $88 \%$ of all patients..$^{14,35-38}$ All parameters reported showed significant improvement compared to the placebo group: a $23-46 \%$ decrease in the number of voids per day, $44-77 \%$ increase in the average voided volume, $56-90 \%$ decrease in incontinence episodes per day, 64-100\% decrease in pads and $39 \%$ increase in maximum cystometric capacity. After 5 years, 121 patients with refractory OAB showed persistence of treatment effect: $84 \%$ of the patients with urgency incontinence and $71 \%$ of the patients with urgency/frequency. ${ }^{14}$ 
In a recent randomized controlled trial, Siegel et al. compared the results of SNM with standard medical therapy (SMT). ${ }^{39}$ Subjects were randomized to SNM $(\mathrm{n}=70)$ and to SMT $(\mathrm{n}=77)$. Patients were included in the SMT group if they failed at least one anticholinergic medication, and had at least one medication that they had not yet attempted. Of all patients, $93 \%$ were female and the mean age was 58 . The intention to treat analysis showed that the success rate at 6 months was significantly greater in the SNM group (61\%) than the SMT group (42\%, $\mathrm{p}=0.02)$. The device-related adverse event rate was $30.5 \%$ and the medication-related adverse event rate was $27.3 \%$.

A randomized crossover trial comparing sacral with pudendal nerve simulation studied 30 patients that all had an electrode implanted at both the sacral nerve $\mathrm{S}_{3}$ and the pudendal nerve. In a blinded, randomized fashion, each lead was tested for 7 days. In total, 24 patients (80\%) showed a successful response to either sacral or pudendal stimulation. The overall reduction in symptoms was $63 \%$ for pudendal and $46 \%$ for SNM. Subjectively, the majority of patients (79\%) preferred pudendal over sacral stimulation. Long-term data have not been published yet. ${ }^{\circ}$

\section{PTNS}

Percutaneous tibial nerve stimulation (PTNS) is a peripheral neuromodulation technique, in which the posterior tibial nerve is stimulated above the medial ankle. The possible working mechanism is neuromodulation at the spinal level. ${ }^{41}$ PTNS requires repeated sessions of stimulation, varying from 6 to 12-weekly episodes. There is evidence of significant improvement in OAB symptoms using PTNS, which is comparable to the effect of antimuscarinics but with a better side effect profile. The SUmiT trial; a multicenter, double-blind, randomized, controlled trial compared the efficacy of PTNS to sham through 12 weeks of therapy..$^{42} \mathrm{~A}$ total of 220 adults with overactive bladder symptoms were randomized to 12 weeks of treatment with weekly PTNS or sham therapy. The PTNS group had statistically significant improvement in frequency, nighttime voids, and urinary urge incontinence episodes compared to sham. No serious device related adverse events were reported. In the OrBIT-trial, 100 adults were randomized to treatment with PTNS to extended-release tolterodine. ${ }^{43}$ The global response assessment demonstrated that subject assessment of overactive bladder symptoms compared to baseline was statistically significant in the PTNS group (79.5\%) compared to the tolterodine group $(54.8 \%, \mathrm{p}=0.01)$.

A review reported improvement in $60-80 \%$ of $\mathrm{OAB}$ patients in incontinence episodes, frequency and urgency44. Others showed that PTNS has a sustained 
safety and efficacy over 36 months with initial success after 12 weekly treatments45. The average number of treatments was 1.1 treatments per month. Although PTNS is considered a safe and effective treatment, long-term results are still lacking. The current NICE guidelines do not recommend PTNS as first-line therapy for the treatment of refractory OAB because of the lack of clinical evidence.

BoNT-A

More than a decade ago, onabotulinum toxin A became a valid option in the treatment of $\mathrm{OAB}$, whether neurogenic or non-neurogenic. It is a valid and effective option in refractory non-neurogenic OAB. As recently reviewed, many trials have evaluated the efficacy and safety of BoNT-A in refractory OAB. ${ }^{46}$

A systematic review and meta-analysis found a total of 1,020 subjects studied in 12 randomized controlled trials. Botulinum toxin-A was associated with less urinary frequency, less incontinence episodes and improvement in quality of life. The most common adverse events were high post-void residual (necessitating (IC) and higher urinary tract infection. Doses of 100-300 U were not consistently differentiated from each other in voiding diaries, quality of life and urge urinary incontinence. No statistically significant difference was seen between bladder body and bladder base, bladder body and bladder body plus trigone in urinary retention and urinary tract infection. ${ }^{47}$

Evaluating a group of 85 patients with refractory OAB who received BoNT-A it was found that patients with intolerance to side effects of antimuscarinic therapy were more likely to respond to BoNT-A than patients who experienced insufficient effect. Patients with intolerance had an $86 \%$ success rate as opposed to $60 \%$ of patients with inefficacy. This finding might indicate that patients who respond to antimuscarinics are more suitable candidates for BoNT-A. ${ }^{48}$ Yet, the small sample size and retrospective nature of this study demands further investigation in a larger cohort. It would be interesting to evaluate if this response is a consistent finding in all patients with OAB refractory to antimuscarinics.

\section{Comparative trials}

Few studies have directly compared third-line treatment strategies for OAB. In chapter 6 we described the results of SNM in 20 patients who were initially treated with BoNT-A. ${ }^{49}$ Most of these patients had discontinued BoNT-A due to a lack of efficacy $(n=17)$ and some patients requested a more permanent solution despite good results $(n=3)$. The mean interval between the botulinum toxin- $A$ and the SNM test stimulation was 23 months. In 14 patients (70\%) the test stim- 
ulation was successful and they were implanted with a device.

In 2012, the ROSETTA trial was started. In this randomized, open-label trial, the results of 200 units BoNT-A are compared with SNM in patients with refractory urgency incontinence..$^{\circ}$ Recently the first result were presented. A total of 386 women were randomly assigned; 369 were treated, and 364 were available for the primary outcome analyses. The onabotulinumtoxinA group reported significantly greater mean reduction in incontinence episodes per day compared to the neuromodulation group. The onabotulinumtoxinA group was significantly more likely to experience complete resolution of urgency incontinence, report greater improvements in overactive bladder symptom bother. Urinary tract infections were higher in the onabotulinumtoxin A group (35\% vs.11\%, p<0.001). Self-catheterization was required in $8 \%$ and $2 \%$ of the onabotulinumtoxin A group at one and six months, respectively and neuromodulation device revisions/removals occurred in $3 \% .^{51}$

\section{Concomitant Fecal and Urinary Symptoms}

Functional disorders of the lower urinary tract often coexist with defecation disorders, pelvic pain or sexual dysfunction. Anatomically, the bladder, anorectum and reproductive organs are closely related. Different factors can be involved, such as neurogenic, mechanical or systemic disease. In this thesis we evaluated the use of SNM in patients with combined urinary and defecation symptoms. In total, 105 patients underwent a test stimulation, of which 59 (56\%) were implanted with a definitive system. Of the implanted patients, 39 had a successful result for only urinary symptoms, 15 had a successful result for both symptoms and 2 only for their fecal symptoms. Compared to botulinum-toxin, SNM has the advantage that both micturition and defecation can improve with the same therapy. Long term results with SNM for fecal incontinence and constipation range from 54 to $63 \% .5^{2}$ Also, SNM has proven to be effective in certain patients with chronic pelvic pain. ${ }^{33}$ Some studies evaluated the results of SNM in patients with combined urinary and fecal symptoms. ${ }^{54,55}$ El-Gazzaz et al. reported that $40 \%$ of all patients treated had a significant improvement at a mean follow up of 24 months. Caremel et al. conducted a survey among patients with double incontinence. They interviewed 37 patients who were treated with SNM (average 30 months since implantation) about their urinary and fecal complaints. In

total, $49 \%$ reported improvement in both complaints. ${ }^{56}$ Although the working mechanism of PTNS is essentially the same as in SNM, the evidence of PTNS in the treatment of functional bowel disorders is less convincing. Recently a large randomized controlled trial showed no significant clinical benefit of PTNS over 
sham electrical stimulation in the treatment of adults with fecal incontinence. ${ }^{57}$ Thin et al. conducted a randomized clinical trial of SNM vs PTNS for fecal incontinence. Although both treatments showed clinical effect, the patients in the SNM group experienced fewer incontinence episodes to the PTNS group.

Therefore, in patients with combined symptoms, SNM might be the preferred treatment. Yet, the degree of bother from each symptom must be taken into consideration, and the focus of treatment should initially be on the most debilitating symptom.

\section{Patient Reference}

Although patient satisfaction with refractory OAB treatments has been studied, little research has been done to discover what patients prefer when considering a refractory treatment for $\mathrm{OAB}$, and which factors influence their decision-making. In studies focusing on SNM, temporary loss of efficacy, discomfort at the implant site, the battery replacement procedure, medical need for magnetic resonance imaging (MRI), and concerns about passing through metal detectors were found to be associated with patient satisfaction. ${ }^{8}$ With respect to Botox, the need for repeated injections, potential need for self-catheterization, and side effects were found to be associated with patient satisfaction..$^{59}$ Hashim et al conducted a survey among patients with refractory $\mathrm{OAB}$, and asked about their preference for third-line treatment, scaling via best-worst, focusing on treatment characteristics. ${ }^{60}$ Among these patients, $57 \%$ chose PTNS, $34 \%$ chose SNM, and $9.4 \%$ chose Botox as their most preferred option. Nevertheless, over $80 \%$ of the patients reported that they would be willing to try each of the options. In contrast, Balchandra et al ${ }^{61}$ who compared preferences for Botox versus SNM among 50 women in the UK, found that $74 \%$ preferred Botox and $26 \%$ preferred SNM. From these conflicting results it seems that the way physicians counsel their patients has an impact on the treatment preference. Since PTNS is the least invasive procedure of the three, most patients probably choose this treatment as a first option. Nevertheless, patient preference is an important factor in the decision making process and this should always be taken into account when commencing a third-line treatment for OAB.

\section{Cost-Effectiveness}

The main goal in dealing with refractory $\mathrm{OAB}$ is to give the best quality of life to each patient at the lowest possible cost for a National Health Care System. The preferred methodology for an economic evaluation is costutility analysis, and the calculation of the incremental cost per additional quality adjusted life year 
(QALY) gained. Various studies have previously evaluated the cost-effectiveness of third-line treatments in both Europe ${ }^{62,63}$ and North America. ${ }^{64,65}$ Most studies suggested that SNM is cost-effective in the medium and long-term compared to BoNT-A. Two recent studies also evaluated the cost-effectiveness of SNM compared to other therapies. Bertapelle et al. conducted a cost-utility analysis of the Italian healthcare system. ${ }^{66}$ They showed that initiating treatment with SNM appears to be cost effective from year three onwards and becomes dominant (i.e. more effective and less costly) at year ten: cumulative costs were $€ 32,975$ for early SNM and $€_{33,309}$ for early BoNT-A, while cumulative QALYs were 7.52 and 6.93, respectively. Autiero et al. compared SNM with percutaneous nerve evaluation (PNE) or tined-lead evaluation (TLP) with optimal medical therapy, BoNT-A and PTNS in the UK. ${ }^{67}$ At 5 years, SNM (PNE or TLP) was more effective and less costly than PTNS. Compared with ongoing medical therapy at 10 years, SNM (PNE or TLP) was more costly and more effective, and compared with BoNT-A, SNM with PNE was less costly and more effective, and SNM with TLE was more costly and more effective.

SNM seems to be both cost saving and more effective, or acceptably cost-effective compared to ongoing medical therapy, PTNS or BoNT-A. The costs for SNM mainly involve device acquisition and implantation. All other treatments involve on-going drug costs and physician visits for maintenance treatment. However, it has to be notified that most study groups received financial support by Medtronic, which possibly introduces a bias. Furthermore, all studies used a simulation model (Markov) which does not fully represent clinical practice. Perhaps the awaited ROSETTA trial, the first randomized, open-label 2-year study designed to compare the effectiveness and cost-effectiveness of BoNT-A and SNM, will provide a direct comparison of these competing treatment options..$^{50}$ As mentioned the first results show that the onabotulinumtoxin A group was significantly more likely to experience complete resolution of urgency incontinence and report greater improvements in overactive bladder symptom bother, but as these are only short term results it is too early for any hard conclusions. ${ }^{.1}$

To summarize, multiple minimally invasive treatments are available for the treatment of refractory $\mathrm{OAB}$, with none showing a strong superiority over another at this time. If the results of one therapy are not satisfactory, switching to another third-line treatment can be attempted. The treatment algorithm is dependent on several factors, including age, comorbidity, patient preference, surgical expertise and financial concerns. All these factors should be taken into consideration before initiation of treatment. 


\section{4 \\ FUTURE PERSPECTIVES}

Although SNM is an established treatment with a good long-term efficacy, there are still various aspects that can be improved. After implantation, some patients lose the therapeutic benefit of SNM. In patients with technical failure (e.g. lead migration or device malfunctioning), treatment effect can often be restored. Yet, approximately $30 \%$ of patients experience treatment failure despite interventions or re-programming of the device. ${ }^{36}$ Furthermore, the number of adverse events, such as device or stimulation related pain symptoms is relatively high, with a reported rate of $27 \% .^{3}$ In the future, procedural and technical modifications for SNM should be anticipated. In our view, the improvement of SNM should be targeted at:

- Increase in treatment efficacy

- Improvement of patient selection

- Increase of patient comfort

- Reduction of adverse events and re-interventions

\section{Patient Selection}

Electrodiagnostic monitoring techniques seem to be promising, since they may allow better patient selection and increase the success rate of test stimulation. ${ }^{68,69}$ Pudendal nerve stimulation and laparoscopic implantation of neuroprotheses can be considered as a conceptual extension of SNM..,71 $\mathrm{CT}$ or $3 \mathrm{D}$ fluoroscopy guidance has been suggested for complex cases, such as spina bifida, other morphological anomalies of the sacral foramina, revision surgery or obese patients. ${ }^{72,73}$ However, for the vast majority of patients no additional benefit is expected. The use is further limited by the lack of sensitivity. Edlund et al. demonstrated that 'adequate sensations', indicating an electrode position in the $\mathrm{S}_{3}$ or $\mathrm{S}_{4}$ foramen, were obtained with a surprisingly wide variety of electrode locations, as shown by x-ray and computed tomography.74

In a randomized study it has been shown that the tined lead can be bent 
along the nerve with a curved stylet, thereby achieving activation of more nerve fibers and motor response at significantly lower voltages in the deepest poles than with the conventional technique. ${ }^{75}$ The authors suggested that battery life could be maximized and programming facilitated. However it has to be proven in further trials, whether these findings will translate into a significantly improved clinical outcome.

Electrodiagnostic techniques during intraoperative lead placement may further improve patient selection and success rates, especially for urological indications, where a more specific urological marker has been discussed. Benson could improve his test success rates significantly up to $80 \%$ in a small series of patients by monitoring urethral response with compound muscle action potentials (CMAP), measured by ring electrodes located on a Foley catheter which had been inserted into the urethra. ${ }^{68}$ In 7 out of 15 patients the position of the electrode relative to the nerve was changed based on the amplitude of the CMAP rather than on the usual biological motor or sensory response. Six of these 7 patients had similar motor/sensory responses, so this alone would not lead to change in nerve site selection. Sensory responses were unreliable; a positive response to testing was obtained in 5 patients who did not have a rectal or vaginal localization of the sensory response.

The LION Procedure (Laparoscopic Implantation of Neuroprothesis) allows direct access to pelvic nerves, thereby having a broad treatment potential. However, the procedure which requires advanced surgical skills, could result in accidental nerve damage due to the nerve diameter in micrometer range and is still at an experimental stage.

The method of two-dimensional pelvic intraoperative neuromonitoring of urinary bladder and internal anal sphincter innervation was found to accurately predict functional results following low anterior resection for rectal cancer. ${ }^{76}$ This novel approach was recently combined with SNM in order to improve lead placement under additional observation of the autonomic innervation. ${ }^{69}$ Thereby, it may further elucidate the mechanism of action and possibly reveal the underlying neuro-physiological phenomena involved during SNM. Matzel et al. already assumed over 20 years ago that the autonomic nervous system to be affected reflexively by SNM." Moreover SNM may give further insight into the truths and myths of nerve sparing pelvic surgery.

Ambulatory-UDS could be a helpful instrument In selecting good candidates for the treatment of a hypocontractile or a-contractile bladder with SNM. Drossearts et al showed that in this group an ambulatory-UDS proves to be better to identify patients with a real acontractile bladder and predicting SNM failure. ${ }^{18}$ 


\section{Patient Comfort}

Patient comfort has improved considerably over the years by a change from open surgery to a minimal invasive procedure and the change from abdominal pacemaker placement to the buttock region. A smaller device, the Interstim II, was also a positive improvement in this respect. We suggest as a potential next step an even smaller, rechargeable device, which makes the need for an IPG replacement, once the battery is empty, obsolete.

\section{Conditional Stimulation}

Intermittent or 'on-demand' stimulation may be an effective alternative to continuous stimulation to reduce stimulation time while preserving efficacy of continence control. In chapter 5 we studied the on-demand use of SNM. We concluded that on-demand neuromodulation is a feasible therapy regimen in the majority of patients with urgency incontinence successfully treated with SNM therapy. Patients can experience more autonomy with on-demand stimulation because they gain full control over the device and are able to tailor the amount of stimulation to their needs. In addition, on-demand stimulation can prolong battery life, postponing replacement of the implantable stimulator. However, inhibitory stimulation must ideally be delivered soon after an overactive contraction begins in order to be effective. Conditional stimulation applied only during bladder contractions, may be more effective than continuous or on-demand stimulation by minimizing habituation of spinal reflexes. ${ }^{78}$ Horvath et al. used EMG of the external urethral sphincter to detect overactive bladder contractions and trigger conditional stimulation of the dorsal genital nerve in patients with spinal cord injury. ${ }^{79}$ Using this closed-loop system, a stimulator was turned on automatically for $20 \mathrm{~s}$ after detection of an overactive detrusor contraction. The EMG measurement reliably detected overactive contractions (threshold $>10 \mathrm{~cm} \mathrm{H}_{2} \mathrm{O}$ ) and could be successfully used as a real-time feedback signal. Although conditional stimulation in this study did not show a significant difference in maximum cystometric capacity increase compared to continuous stimulation, it significantly reduced stimulation time. These results may support future efforts in developing a neural prosthesis for closed-loop electrical control of urinary continence.

\section{Expanding Indications}

Interstitial cystitis (IC) is a condition characterized by symptoms of urinary frequency, urgency and pelvic pain. Pharmacotherapy is the current mainstay 
of treatment, but is inadequate for many. However, the urinary symptoms are FDA approved indications for SNM, and chronic pelvic pain is not. In a study of 21 refractory IC patients treated with SNM, subjective pain was reported to be improved and additionally, it decreased narcotic usage at a mean of 15.4 months follow-up. ${ }^{80}$ Chronic pelvic pain in the absence of urinary symptoms shows to be a difficult problem to treat. Often it presents with a variety of symptoms and affects multiple systems and for that reason no standard treatment can be applied. ${ }^{81}$

SNM was evaluated in one series of ten patients with chronic, refractory pelvic pain without significant voiding symptoms. Improvement of at least $40 \%$ after PNE qualified patients for a permanent implant. Median follow-up was 19 months (6-74 months) after implantation. Six out of 10 patients experienced a substantial benefit. SNM decreased subjective pain severity and the number of hours with pain..$^{82}$ In a review of 10 studies evaluating SNM in patients with chronic pelvic pain, the success rate was reported to be between 60 and $77 \% .53$ The mean reduction in pain symptoms ranged between 27 and 50\%. However, there is currently insufficient data to determine the role of SNM in the treatment of chronic pelvic pain. Because the results seem to be promising, larger prospective trials with long-term evaluation are recommended.

SNM has also been widely used for functional bowel disorders. Fecal incontinence is currently treated with medications, pelvic floor biofeedback and surgery. The role of SNM has been explored by Altomare et al. in a multicenter study of 94 fecal incontinence patients. $63 \%$ of these patients went on to have permanent lead placement and $87 \%$ had long term follow-up (mean 74 months). Three-quarters of the patient population maintained at least $50 \%$ improvement after more than five years. They showed significant improvements in physical, social and emotional functioning on QOL domains. Manometric data for resting and squeeze anal pressure significantly improved. Urgency sensation and maximal volume tolerated showed a trend towards reduction. ${ }^{83}$ Two recent reviews confirmed the efficacy and safety, with positive results in the longterm. ${ }^{84,85}$ SNM has also been successfully used in the treatment of constipation. Ten studies evaluated the results of SNM in patients with constipation. ${ }^{86}$ A total of 225 temporary neuromodulation tests and 125 permanent implants were performed. Bowel diaries showed improvement in the assessment criteria in more than $50 \%$ of patients on temporary neuromodulation and the results were maintained in approximately $90 \%$ of patients who underwent a permanent implantation over medium to long-term follow-up (median follow up ranged from 11 to 42 months). SNM might also be a promising treatment for irritable 
bowel syndrome. In a randomized cross-over trial of 20 patients Fassov et al. showed that SNM changes rectal sensitivity. SNM reduced wall stiffness and increased sensitivity to stretch, which are associated with an improved irritable bowel symptom score87. In the same group of patients, IBS-specific symptom and quality of life scores were significantly reduced during stimulation. ${ }^{88} \mathrm{At}$ 1-year follow-up, the median IBS-specific symptom score (25; range, 13-65) was significantly lower than that at baseline (62; range, 45-80) $(\mathrm{p}=0.0001)$.

Dysfunctional elimination syndrome in children includes disturbances of the GI and urinary tract. Dwyer et al. studied 105 patients with symptoms refractory to medical therapy. ${ }^{89}$ The mean follow-up was 2.7 years. SNM showed improvement in urinary incontinence (88\%), constipation (79\%), frequency and/or urgency (67\%), and nocturnal enuresis (66\%) of all patients. Groen et al. showed success of SNM in 18 children with OAB and voiding dysfunction due to Fowler syndrome..$^{90}$ An initial full response was achieved in 9 out of 18 patients (50\%) and partial response in $5(28 \%)$. After a mean follow-up of 28 months, 6 out of 15 patients (40\%) had a full response and $5(33 \%)$ had a partial response. Although the results of SNM in the pediatric population seem promising, both the efficacy 


\section{5 \\ CONCLUSION}

SNM is a safe and effective third-line treatment option for patients with refractory $\mathrm{OAB}$ and non-obstructive urinary retention. Advances in implantation technique and improvement of materials have made SNM a minimally-invasive and upfront treatment. The current role of SNM in the management of refractory $O A B$ is debatable and the choice of treatment is dependent on multiple factors including age, comorbidity, patient preference, surgical expertise and resources. Future studies will hopefully elucidate the exact position in the algorithm of different treatment strategies and cost-effectiveness. Moreover, future research should focus on further improvement of existing and development of new neuromodulation techniques, improvements in the selection of optimal candidates or development of customized approaches and a further increase in patient comfort. 


\section{6 REFERENCES}

1 Tanagho EA, Schmidt RA. Bladder pacemaker: scientific basis and clinical future. Urology 1982;20:614-9.

2 Spinelli M, Giardiello G, Gerber M, Arduini A, van den Hombergh U, Malaguti S. New sacral neuromodulation lead for percutaneous implantation using local anesthesia: description and first experience. J Urol 2003;170:1905-7.

3 Marcelissen TA, Leong RK, de Bie RA, van Kerrebroeck PE, de Wachter SG. Long-term results of sacral neuromodulation with the tined lead procedure. J Urol 2010;184:1997-2000.

4 Spinelli M, Sievert KD. Latest technologic and surgical developments in using InterStim Therapy for sacral neuromodulation: impact on treatment success and safety. Eur Urol 2008;54:1287-96.

5 Janknegt RA, Weil EH, Eerdmans PH. Improving neuromodulation technique for refractory voiding dysfunctions: two-stage implant. Urology 1997;49:358-62.

6 Leong RK, De Wachter SG, Nieman FH, de Bie RA, van Kerrebroeck PE. PNE versus 1st stage tined lead procedure: a direct comparison to select the most sensitive test method to identify patients suitable for sacral neuromodulation therapy. Neurourol Urodyn 2011;30:1249-52.

7 Marcelissen T, Leong R, Serroyen J, van Kerrebroeck PE, de Wachter S. Is the screening method of sacral neuromodulation a prognostic factor for long-term success? J Urol 2011;185:583-7.

8 Scheepens WA, Jongen MM, Nieman FH, de Bie RA, Weil EH, van Kerrebroeck PE. Predictive factors for sacral neuromodulation in chronic lower urinary tract dysfunction. Urology 2002;60:598-602.

9 Marcelissen TA, Leong RK, Nieman FH, van Lankveld JJ, van Kerrebroeck PE, de Wachter SG. Psychological and psychiatric factors as predictors for success in sacral neuromodulation treatment. BJU Int 2011;108:1834-8.

10 Drossaerts J, Vrijens D, Leue C, Schilders I, van Kerrebroeck PE, van Koeveringe G. Screening for depression and anxiety in patients with storage or voiding dysfunction: A retrospective cohort study predicting outcome of sacral neuromodulation. Neurourol Urodyn 2015.

11 Peters KM, Killinger KA, Boura JA. Is sensory testing during lead placement crucial for achieving positive outcomes after sacral neuromodulation? Neurourol Urodyn 2011;30:148992.

12 Everaert K, Plancke H, Lefevere F, Oosterlinck W. The urodynamic evaluation of neuromodulation in patients with voiding dysfunction. Br J Urol 1997;79:702-7.

13 Scheepens WA, van Koeveringe GA, de Bie RA, Weil EH, van Kerrebroeck PE. Urodynamic results of sacral neuromodulation correlate with subjective improvement in patients with an overactive bladder. Eur Urol 2003;43:282-7.

14 Hassouna MM, Siegel SW, Nyeholt AA, et al. Sacral neuromodulation in the treatment of urgency-frequency symptoms: a multicenter study on efficacy and safety. J Urol 2000;163:184954 . 
15 Weil EH, Ruiz-Cerda JL, Eerdmans PH, Janknegt RA, Bemelmans BL, van Kerrebroeck PE. Sacral root neuromodulation in the treatment of refractory urinary urge incontinence: a prospective randomized clinical trial. Eur Urol 2000;37:161-71.

16 DasGupta R, Fowler CJ. Urodynamic study of women in urinary retention treated with sacral neuromodulation. J Urol 2004;171:1161-4.

17 Jonas U, Fowler CJ, Chancellor MB, et al. Efficacy of sacral nerve stimulation for urinary retention: results 18 months after implantation. J Urol 2001;165:15-9.

18 Drossaerts J, Rademakers K, van Koeveringe G, Van Kerrebroeck PE. The value of urodynamic tools to guide patient selection in sacral neuromodulation. World J Urol 2015;33:1889-95.

19 Scheepens WA, de Bie RA, Weil EH, van Kerrebroeck PE. Unilateral versus bilateral sacral neuromodulation in patients with chronic voiding dysfunction. J Urol 2002;168:2046-50.

20 Marcelissen TA, Leong RK, Serroyen J, van Kerrebroeck PE, De Wachter SG. The use of bilateral sacral nerve stimulation in patients with loss of unilateral treatment efficacy. J Urol 2011;185:976-80.

21 Marcelissen TA, Leong RK, Nieman FH, de Bie RA, van Kerrebroeck PE, de Wachter SG. The effect of pulse rate changes on the clinical outcome of sacral neuromodulation. J Urol 2011;185:1781-5.

22 Peters KM, Shen L, McGuire M. Effect of Sacral Neuromodulation Rate on Overactive Bladder Symptoms: A Randomized Crossover Feasibility Study. Low Urin Tract Symptoms 2013;5:12933.

23 Thomas GP, Duelund-Jakobsen J, Dudding TC. A double-blinded randomized multicentre study to investigate the effect of changes in stimulation parameters on sacral nerve stimulation for constipation. Colorectal Dis 2015;17:990-5.

24 Novara G, Galfano A, Secco S, et al. A systematic review and meta-analysis of randomized controlled trials with antimuscarinic drugs for overactive bladder. Eur Urol 2008;54:740-63.

25 Buser N, Ivic S, Kessler TM, Kessels AG, Bachmann LM. Efficacy and adverse events of antimuscarinics for treating overactive bladder: network meta-analyses. Eur Urol 2012;62:104060.

26 Kessler TM, Bachmann LM, Minder C, et al. Adverse event assessment of antimuscarinics for treating overactive bladder: a network meta-analytic approach. PLoS One 2011;6:e16718.

27 Khullar V, Chapple C, Gabriel Z, Dooley JA. The effects of antimuscarinics on health-related quality of life in overactive bladder: a systematic review and meta-analysis. Urology 2006;68:38-48.

28 Leone Roberti Maggiore U, Cardozo L, Ferrero S, et al. Mirabegron in the treatment of overactive bladder. Expert Opin Pharmacother 2014;15:873-87.

29 Wagg A, Cardozo L, Nitti VW, et al. The efficacy and tolerability of the beta3-adrenoceptor agonist mirabegron for the treatment of symptoms of overactive bladder in older patients. Age Ageing 2014;43:666-75.

30 Chapple CR, Cardozo L, Nitti VW, Siddiqui E, Michel MC. Mirabegron in overactive bladder: a review of efficacy, safety, and tolerability. Neurourol Urodyn 2014;33:17-30.

31 Abrams P, Kelleher C, Staskin D, et al. Combination treatment with mirabegron and solifenacin in patients with overactive bladder: efficacy and safety results from a randomised, double-blind, dose-ranging, phase 2 study (Symphony). Eur Urol 2015;67:577-88.

32 Yamaguchi O, Kakizaki H, Homma Y, et al. Safety and efficacy of mirabegron as 'add-on' therapy in patients with overactive bladder treated with solifenacin: a post-marketing, open-label study in Japan (MILAI study). BJU Int 2015;116:612-22.

33 Bartley J, Gilleran J, Peters K. Neuromodulation for overactive bladder. Nat Rev Urol 2013;10:513-21. 
34 Herbison GP, Arnold EP. Sacral neuromodulation with implanted devices for urinary storage and voiding dysfunction in adults. Cochrane Database Syst Rev 2009:CDoo4202.

35 Sutherland SE, Lavers A, Carlson A, Holtz C, Kesha J, Siegel SW. Sacral nerve stimulation for voiding dysfunction: One institution's 11-year experience. Neurourol Urodyn 2007;26:19-28; discussion 36 .

36 van Kerrebroeck PE, van Voskuilen AC, Heesakkers JP, et al. Results of sacral neuromodulation therapy for urinary voiding dysfunction: outcomes of a prospective, worldwide clinical study. J Urol 2007;178:2029-34.

37 Bosch JL, Groen J. Sacral nerve neuromodulation in the treatment of patients with refractory motor urge incontinence: long-term results of a prospective longitudinal study. J Urol 2000;163:1219-22.

38 Siegel SW, Catanzaro F, Dijkema HE, et al. Long-term results of a multicenter study on sacral nerve stimulation for treatment of urinary urge incontinence, urgency-frequency, and retention. Urology 2000;56:87-91.

39 Siegel S, Noblett K, Mangel J, et al. Results of a prospective, randomized, multicenter study evaluating sacral neuromodulation with InterStim therapy compared to standard medical therapy at 6-months in subjects with mild symptoms of overactive bladder. Neurourol Urodyn 2015;34:224-30.

40 Peters KM, Feber KM, Bennett RC. Sacral versus pudendal nerve stimulation for voiding dysfunction: a prospective, single-blinded, randomized, crossover trial. Neurourol Urodyn 2005;24:643-7.

41 Pal F, Heesakkers JP, Bemelmans LH. Current opinion on the working mechanisms of neuromodulation in the treatment of lower urinary tract dysfunction. Current Opinion in Urology 2006;2006:261-7.

42 Peters KM, Carrico DJ, Perez-Marrero RA, et al. Randomized trial of percutaneous tibial nerve stimulation versus Sham efficacy in the treatment of overactive bladder syndrome: results from the SUmiT trial. J Urol 2010;183:1438-43.

43 Peters KM, Macdiarmid SA, Wooldridge LS, et al. Randomized trial of percutaneous tibial nerve stimulation versus extended-release tolterodine: results from the overactive bladder innovative therapy trial. J Urol 2009;182:1055-61.

44 Biemans JM, van Balken MR. Efficacy and effectiveness of percutaneous tibial nerve stimulation in the treatment of pelvic organ disorders: a systematic review. Neuromodulation 2013;16:25-33; discussion

45 Peters KM, Carrico DJ, Wooldridge LS, Miller CJ, MacDiarmid SA. Percutaneous tibial nerve stimulation for the long-term treatment of overactive bladder: 3-year results of the STEP study. J Urol 2013;189:2194-201.

46 Mangera A, Apostolidis A, Andersson KE, et al. An updated systematic review and statistical comparison of standardised mean outcomes for the use of botulinum toxin in the management of lower urinary tract disorders. Eur Urol 2014;65:981-90.

47 Cui Y, Wang L, Liu L, et al. Botulinum toxin-A injections for idiopathic overactive bladder: a systematic review and meta-analysis. Urol Int 2013;91:429-38.

48 Makovey I, Davis T, Guralnick ML, O'Connor RC. Botulinum toxin outcomes for idiopathic overactive bladder stratified by indication: lack of anticholinergic efficacy versus intolerability. Neurourol Urodyn 2011;30:1538-40.

49 Smits MA, Oerlemans DJ, Marcelissen TA, Van Kerrebroeck PE, De Wachter SG. Sacral neuromodulation in patients with idiopathic overactive bladder after initial botulinum toxin therapy. J Urol 2013;190:2148-52. 
50 Amundsen CL, Richter HE, Menefee S, et al. The Refractory Overactive Bladder: Sacral NEuromodulation vs. BoTulinum Toxin Assessment: ROSETTA trial. Contemp Clin Trials 2014;37:272-83.

51 C.L. A, Richter HE, Menefee SA, et al. Sacral Neuromodulation versus OnabotulinumtoxinA for Refractory Overactive Bladder J Urol 2016;195:e949.

52 Thin NN, Horrocks EJ, Hotouras A, et al. Systematic review of the clinical effectiveness of neuromodulation in the treatment of faecal incontinence. British Journal of Surgery 2013;100:1430-47.

53 Marcelissen T, Jacobs R, van Kerrebroeck PE, de Wachter S. Sacral neuromodulation as a treatment for chronic pelvic pain. J Urol 2011;186:387-93.

54 Uludag O, Melenhorst J, Koch SM, van Gemert WG, Dejong CH, Baeten CG. Sacral neuromodulation: long-term outcome and quality of life in patients with faecal incontinence. Colorectal Dis 2011;13:1162-6.

55 El-Gazzaz G, Zutshi M, Salcedo L, Hammel J, Rackley R, Hull T. Sacral neuromodulation for the treatment of fecal incontinence and urinary incontinence in female patients: long-term follow-up. Int J Colorectal Dis 2009;24:1377-81.

56 Caremel R, Damon H, Ruffion A, et al. Can sacral neuromodulation improve minor incontinence symptoms in doubly incontinent patients successfully treated for major incontinence symptoms? Urology 2012;79:80-5.

57 Knowles CH, Horrocks EJ, Bremner SA, et al. Percutaneous tibial nerve stimulation versus sham electrical stimulation for the treatment of faecal incontinence in adults (CONFIDeNT): a double-blind, multicentre, pragmatic, parallel-group, randomised controlled trial. Lancet 2015; 386:1640-8.

58 Leong RK, Marcelissen TA, Nieman FH, De Bie RA, Van Kerrebroeck PE, De Wachter SG. Satisfaction and patient experience with sacral neuromodulation: results of a single center sample survey. J Urol 2011;185:588-92.

59 Imam SZ, Syed KS, Ali SA, et al. Patients' satisfaction and opinions of their experiences during admission in a tertiary care hospital in Pakistan - a cross sectional study. BMC Health Serv Res 2007;7:161.

60 Hashim H, Beusterien K, Bridges JF, Amos K, Cardozo L. Patient preferences for treating refractory overactive bladder in the UK. Int Urol Nephrol 2015;47:1619-27.

61 Balchandra P, Rogerson L. Women's perspective: intra-detrusor botox versus sacral neuromodulation for overactive bladder symptoms after unsuccessful anticholinergic treatment. Int Urogynecol J 2014;25:1059-64.

62 Arlandis S, Castro D, Errando C, et al. Cost-effectiveness of sacral neuromodulation compared to botulinum neurotoxin a or continued medical management in refractory overactive bladder. Value Health 2011;14:219-28.

63 Leong RK, de Wachter SG, Joore MA, van Kerrebroeck PE. Cost-effectiveness analysis of sacral neuromodulation and botulinum toxin A treatment for patients with idiopathic overactive bladder. BJU Int 2011;108:558-64.

64 Hassouna MM, Sadri H. Economic evaluation of sacral neuromodulation in overactive bladder: A Canadian perspective. Can Urol Assoc J 2015;9:242-7.

65 Siddiqui NY, Amundsen CL, Visco AG, Myers ER, Wu JM. Cost-effectiveness of sacral neuromodulation versus intravesical botulinum A toxin for treatment of refractory urge incontinence. J Urol 2009;182:2799-804.

66 Bertapelle MP, Vottero M, Popolo GD, et al. Sacral neuromodulation and Botulinum toxin A for refractory idiopathic overactive bladder: a cost-utility analysis in the perspective of Italian Healthcare System. World J Urol 2015;33:1109-17. 
67 Autiero SW, Hallas N, Betts CD, Ockrim JL. The cost-effectiveness of sacral nerve stimulation (SNS) for the treatment of idiopathic medically refractory overactive bladder (wet) in the UK. BJU Int 2015;116:945-54.

68 Benson JT. Sacral nerve stimulation results may be improved by electrodiagnostic techniques. Int Urogynecol J Pelvic Floor Dysfunct 2000;11:352-7.

69 Kneist W, Kauff DW, Schroder M, Koch KP, Lang H. Percutaneous nerve evaluation based on electrode placement under control of autonomic innervation. Tech Coloproctol 2014;18:725-30.

70 Spinelli M, Malaguti S, Giardiello G, Lazzeri M, Tarantola J, Van Den Hombergh U. A new minimally invasive procedure for pudendal nerve stimulation to treat neurogenic bladder: description of the method and preliminary data. Neurourol Urodyn 2005;24:305-9.

71 Possover M, Baekelandt J, Chiantera V. The Laparoscopic Implantation of Neuroprothesis (LION) Procedure to Control Intractable Abdomino-Pelvic Neuralgia. Neuromodulation 2007;10:18-23.

72 Amoroso L, Pelliccioni G, Ghiselli R, Scarpino O, Saba V, Ricci S. Sacral-neuromodulation CT-guided. Radiol Med 2005;109:421-9.

73 Hellstrom PA, Katisko J, Finnila P, Vaarala MH. Sacral nerve stimulation lead implantation using the O-arm. BMC Urol 2013;13:48.

74 Edlund C, Hellstrom M, Peeker R, Fall M. First Scandinavian experience of electrical sacral nerve stimulation in the treatment of the overactive bladder. Scand J Urol Nephrol 2000;34:366-76.

75 Jacobs SA, Lane FL, Osann KE, Noblett KL. Randomized prospective crossover study of interstim lead wire placement with curved versus straight stylet. Neurourol Urodyn 2014;33:488-92.

76 Kauff DW, Koch KP, Somerlik KH, Hoffmann KP, Lang H, Kneist W. Evaluation of two-dimensional intraoperative neuromonitoring for predicting urinary and anorectal function after rectal cancer surgery. Int J Colorectal Dis 2013;28:659-64.

77 Matzel KE, Schmidt RA, Tanagho EA. Neuroanatomy of the striated muscular anal continence mechanism. Implications for the use of neurostimulation. Dis Colon Rectum 1990;33:666-73.

78 Kirkham AP, Shah NC, Knight SL, Shah PJ, Craggs MD. The acute effects of continuous and conditional neuromodulation on the bladder in spinal cord injury. Spinal Cord 2001;39:420-8.

79 Horvath EE, Yoo PB, Amundsen CL, Webster GD, Grill WM. Conditional and continuous electrical stimulation increase cystometric capacity in persons with spinal cord injury. Neurourol Urodyn 2010;29:401-7.

8o Bernstein A, Peters K. Expanding indications for neuromodulation. Urol Clinics N Am 2005;32:59-63.

81 Quaghebeur J, Wyndaele JJ. Chronic pelvic pain syndrome: role of a thorough clinical assessment. Scand J Urol 2015;49:81-9.

82 Siegel S, Paszkiewicz E, Kirkpatrick C, Hinkel B, Oleson K. Sacral nerve stimulation in patients with chronic intractable pelvic pain. J Urol 2001;166:1742-5.

83 Altomare DF, Ratto C, Ganio E, Lolli P, Masin A, Villani RD. Long-term outcome of sacral nerve stimulation for fecal incontinence. Dis Colon Rectum 2009;52:11-7.

84 Thaha MA, Abukar AA, Thin NN, Ramsanahie A, Knowles CH. Sacral nerve stimulation for faecal incontinence and constipation in adults. Cochrane Database Syst Rev 2015;8:CDoo4464.

85 Noblett KL, Cadish LA. Sacral nerve stimulation for the treatment of refractory voiding and bowel dysfunction. Am J Obstet Gynecol 2014;210:99-106.

86 Sharma A, Bussen D, Herold A, Jayne D. Review of sacral neuromodulation for management of constipation. Surg Innov 2013;20:614-24. 
87 Fassov J, Brock C, Lundby L, et al. Sacral nerve stimulation changes rectal sensitivity and biomechanical properties in patients with irritable bowel syndrome. Neurogastroenterol Motil 2014;26:1597-604.

88 Fassov JL, Lundby L, Laurberg S, Buntzen S, Krogh K. A randomized, controlled, crossover study of sacral nerve stimulation for irritable bowel syndrome. Ann Surg 2014;260:31-6.

89 Dwyer ME, Vandersteen DR, Hollatz P, Reinberg YE. Sacral neuromodulation for the dysfunctional elimination syndrome: a 10-year single-center experience with 105 consecutive children. Urology 2014;84:911-7.

90 Groen LA, Hoebeke P, Loret N, et al. Sacral neuromodulation with an implantable pulse generator in children with lower urinary tract symptoms: 15-year experience. J Urol 2012;188:1313-7. 


\section{SUMMARY}

Sacral Neuromodulation (SNM) is an established treatment for patients with refractory lower urinary tract dysfunction. Since the FDA approval in 1997 there have been many advancements in technical and surgical aspects, which have improved treatment safety and efficacy. These developments include the percutaneous tined lead, use of local anesthesia and fluoroscopy, and a smaller implantable stimulator. This thesis gives an overview of the clinical experience with SNM treatment. Clinical experience and continuous research resulted in a better understanding of the application of SNM. This has improved patient selection, the application of this technique and the management of complications during follow-up.

Chapter 1 discusses the different types of lower urinary tract dysfunction. The principle of neuromodulation is discussed and the historical developments and different forms of neurostimulation are described. The technique of sacral neuromodulation and its indications are also explained. Finally the outline of this thesis is presented.

Chapter 2 gives an overview of the current knowledge on neuromodulation. We describe the developments in electrostimulation and neuromodulation, and discuss the evolution of neuromodulation techniques, starting with the peripheral nerve evaluation test. Initially a positive test result was followed by an open implantation, and this evolved into a 2-phase procedure. Later the minimally invasive tined lead was introduced and the open procedure was abandoned. In most patients, the 3rd sacral root is stimulated during SNM. Success rates are presented and vary between 60 to $80 \%$. Most seen complications are lead migration, pain at implant side and infection. Finally the expanding indications as the treatment of neurogenic lower urinary tract dysfunction, the treatment of chronic bladder and pelvic pain and the treatment of bowel dysfunctions are discussed.

Chapter 3 reviews the results of our analysis studying the influence of tech- 
nical improvements in SNM and the long-term results in our clinic. We concluded that SNM provides lasting benefit in patients with refractory symptoms of overactive bladder and non-obstructive urinary retention. The improvement in outcome and reduction of reoperation rate can be attributed to the learning curve and technical improvements.

Chapter 4 describes our medium term results with the tined lead in 49 patients. The tined lead made it possible to perform the procedure under local anesthesia and it can be used as a test stimulation. We concluded that test stimulation with the tined lead is more reliable than the open procedure and that the clinical efficacy is high and durable.

Chapter 5 presents the results of a study on the feasibility to use SNM in an on demand setting. Intermittent stimulation may enable an increase in battery life and may postpone loss of efficacy in the long term. We observed that intermittent stimulation gave no worsening in symptoms in the majority of patients. An additional benefit was that several patients felt more in control over their voiding symptoms with on demand stimulation.

Chapter 6 evaluates whether patients with overactive bladder and incontinence who discontinued intravesical botulinum toxin A therapy can be successfully treated with SNM. We concluded that the success rate after botulinum A therapy was comparable with the group of patients without a history of botulinum toxin-A treatment.

Chapter 7 presents our experiences with SNM in patients with combined micturition and defecation disorders. We included all patients from our Pelvic Care Center Maastricht with combined disorders that were treated with SNM between 2008 and 2013. We concluded that $25 \%$ of the implanted patients showed $>50 \%$ improved for both urinary and fecal symptoms.

Chapter 8 is the general discussion. We present an overview of the most important improvements in the past, the role of SNM compared to other third-line treatments at present, and the role of SNM and opportunities for improvement in the future. After the introduction of SNM by Tanagho, the procedure was complex and time consuming, but during the 1990's technical improvements were made resulting in better clinical results. In 2002 the tined lead was introduced and made a percutaneous technique possible. Predictive factors for selecting good candidates were studied in different settings. Psychological problems might have an effect on the outcome.

Urodynamic studies revealed that with SNM an increase in bladder contraction, an enhancement of sphincter relaxation and a decrease in bladder capacity can be achieved. Recently a study on ambulatory urodynamics showed that the 
presence of bladder contractility is of importance for the success of SNM treatment.

We conclude that in the future, procedural and technical modifications in SNM should be anticipated. In our view, the improvement of SNM should be targeted at increase in treatment efficacy, improvement of patient selection, increase of patient comfort and reduction of adverse events. Ambulatory urodynamics could be a helpful instrument in selecting good candidates. Electro-diagnostic techniques during intraoperative lead placement may further improve patient selection and success rates. Intermittent therapy should be considered to increase battery life and can give patients an increased bodily control. A rechargeable device can further improve patient comfort.

In summary, SNM is a safe and effective third-line treatment option for patients with refractory $\mathrm{OAB}$ and non-obstructive urinary retention. Many improvements made SNM a minimally invasive and successful treatment. Future research should focus on further improvement of existing, and development of new techniques, comprehensive selection of optimal candidates and further increase in patient comfort. 


\section{NEDERLANDSE SAMENVATTING}

Dit proefschrift beschrijft de resultaten van de behandeling van chronische blaasklachten met behulp van sacrale neuromodulatie. Chronische blaasklachten zoals een overactieve blaas of niet-obstructieve blaasledigingsklachten vormen vaak een lastig probleem en zijn een uitdaging voor de uroloog. De meeste patiënten worden behandeld met fysiotherapie, medicatie of een combinatie van beide. Als het probleem blijft bestaan, vinden er soms ingrijpende behandelingen plaats zoals operaties om de blaas te vergroten of geheel te vervangen. Dit zijn grote operaties met mogelijk belangrijke complicaties. Dit was een reden om te gaan zoeken naar een minder ingrijpende therapie. De Amerikaanse uroloog Tanagho ontwikkelde eind jaren ' 80 een methode om de sacrale zenuwen te stimuleren bij patiënten met blaasklachten. Er werd aangenomen dat de blaascontrole hersteld kon worden door stimulatie van de zenuwprikkels die richting de hersenen gaan en door modulatie van de blaasreflexen in het centrale zenuwstelsel. De behandeling die hieruit ontstaan is, werd sacrale neuromodulatie (SNM) genoemd.

De techniek van SNM bestaat uit een electrode die gekoppeld is aan een geimplanteerde neurostimulator. In de beginperiode werd deze electrode via een open operatie geplaatst waarbij het heiligbeen werd vrij gelegd. Later is er een methode ontwikkeld om een zelf-verankerende electrode te plaatsen zonder dat het nodig is een incisie te maken, de zogenaamde 'tined lead procedure' (TLP). In dit proefschrift worden de resultaten beschreven van zowel de eerste techniek (hoofdstuk 3) als ook de meer moderne methode (hoofdstuk 4).

Omdat de oorzaak van blaasklachten bij patiënten niet altijd duidelijk is, is het erg lastig geschikte kandidaten voor de behandeling met SNM te selecteren. Om de beste kandidaten te kiezen wordt er voor de definitieve implantatie altijd eerst een proefstimulatie uitgevoerd. In de begin jaren bestond deze proeffase uit een 'Percutaneous Nerve Evaluation' test (PNE), waarbij via een naald de zenuwreactie getest wordt en waarna bij een goede reactie een dunne electrode geplaatst kon worden. Omdat deze electrode zeer fragiel was en zich makkelijk 
verplaatste, wordt deze tegenwoordig tijdens de testfase vaak vervangen door een zelf-verankerende electrode. Dit noemen we de eerste fase van een TLP.

Het doel van dit proefschrift is het beschrijven van de resultaten met sacrale neuromodulatie. De lange termijnresultaten van zowel de oude als de nieuwe techniek zijn geëvalueerd, waarbij gelet is op de effectiviteit, complicaties en patiënt tevredenheid. De behaalde resultaten zijn telkens gerelateerd aan de op dat moment bekende behandelingsmogelijkheden. Omdat er in de loop van de jaren nieuwe indicaties maar ook nieuwe behandelingen bijgekomen zijn, wordt ook hier op ingegaan en werpen we tevens een blik op de toekomst.

Hoofdstuk 1 is een inleidend hoofdstuk en geeft een overzicht van de chronische blaasklachten zoals overactieve blaas en urine incontinentie. Tevens worden de verschillende behandelopties besproken. Ook wordt er een overzicht gegeven van de verschillende vormen van zenuwstimulatie en de tot dusver bekende resultaten. Tenslotte wordt in dit hoofdstuk de opbouw van dit proefschrift gepresenteerd.

Hoofdstuk 2 geeft een overzicht van het ontstaan en de resultaten van SNM. Er wordt beschreven hoe neuromodulatie een ontwikkeling is die voortvloeit uit allerlei andere vormen van neurostimulatie die in de loop van de geschiedenis in de geneeskunde toegepast zijn. Ook wordt beschreven welke ontwikkelingen de implantatietechniek heeft doorgemaakt. Er worden verschillende studies geciteerd die allemaal concluderen dat SNM een veilige en effectieve therapie is. Vervolgens worden enkele nieuwere indicaties beschreven zoals de behandeling van mictieklachten bij patiënten met een neurogene blaas, de behandeling van interstitiële cystitis en de behandeling van darmklachten.

Hoofdstuk 3 beschrijft de lange termijn resultaten van 149 patiënten die vanaf 1990 in het MUMC+ behandeld zijn. Hierbij zit dus de beginperiode van de behandeling met ook de bijbehorende leercurve. Geconcludeerd wordt dat SNM een blijvend effect heeft bij de behandeling van hardnekkige symptomen van overactieve blaas en niet-obstructieve urine retentie. De verbetering van het effect en het verminderen van het aantal complicaties kan toegeschreven worden aan de leercurve en de technische verbeteringen.

In hoofdstuk 4 geeft de resultaten op middellange termijn weer van 49 patienten die middels de 'tined lead' methode behandeld zijn. Geconcludeerd wordt dat er op middellange termijn positieve resultaten te zien zijn, waarbij blijkt dat het testen in 2 fasen meer betrouwbaar is dan de PNE test. De zelf verankerende electrode lijkt bovendien op middellange termijn goed gefixeerd te blijven.

Hoofdstuk 5 bespreekt een pilot-intervention studie waarin onderzocht is of 
het mogelijk is om patiënten die met SNM behandeld worden ook intermitterend (on-demand) te stimuleren. De aanleiding voor deze studie was het verlies van effect dat bij sommige patiënten na een aantal jaren gezien werd en geduid werd als gewenning van het zenuwstelsel. De resultaten tonen aan dat na 2 weken on-demand gebruik er bij de meerderheid van de patiënten een vergelijkbaar effect gezien werd als bij continu stimulatie. Intermittente stimulatie levert meer autonomie op, leidt tot een langere levensduur van de batterij en veroorzaakt minder kans op gewenning van de stimulatie.

In hoofdstuk 6 wordt een studie gepresenteerd met de resultaten van een groep patiënten die behandeld werden met neuromodulatie nadat ze één of meerdere behandelingen met botuline toxine (botox) injecties hebben gehad.

De conclusie van deze studie is dat een behandeling met neuromodulatie met goed resultaat mogelijk is na een behandeling met botox. De resultaten na 1 jaar zijn vergelijkbaar met patiënten die geen behandeling met botox van tevoren hebben gehad.

In hoofdstuk 7 worden de resultaten van een groep patiënten die zowel mictie- als defecatieklachten hebben geëvalueerd. Binnen ons Pelvic Care Centrum Maastricht rapporteert $74 \%$ van de patiënten mictieproblemen en $23 \%$ klaagt over darmklachten. Patiënten die een gecombineerd probleem hadden, werden getest en bij goed resultaat behandeld met sacrale neuromodulatie therapie. Met deze studie is aangetoond dat neuromodulatie een mogelijke therapie is voor mensen met een dubbel probleem. Afhankelijk van de klachten en de etiologie is er een hogere kans op succes mogelijk.

Hoofdstuk 8 is het discussie hoofdstuk waarin we een overzicht geven van belangrijke verbeteringen die in het verleden met sacrale neuromodulatie gerealiseerd zijn. Vervolgens worden de huidige mogelijkheden voor de behandeling van overactieve blaas met deze techniek besproken en met elkaar vergeleken. Tenslotte is aangegeven welke verdere verbeteringen in de toekomst verwacht mogen worden.

Conclusie: SNM is een veilige en effectieve therapie voor patiënten die niet reageren op de standaard therapie voor overactieve blaas en niet-obstructieve urine retentie. In de loop van de jaren zijn er vele verbeteringen doorgevoerd, waardoor SNM een minimaal invasieve behandeling is geworden die goede resultaten oplevert.

Toekomstig onderzoek moet zich richten op de ontwikkeling van nieuwe technieken, op verbetering van patiëntenselectie en op optimalisatie van het patiëntencomfort. 


\section{VALORISATION ADDENDUM}

\section{Introduction}

Chronic lower urinary tract dysfunction (LUTD) is a common disorder that has a significant impact on quality of life. As described in the introduction of this thesis, the term 'dysfunction' refers to an abnormality in the physiology of the lower urinary tract, including the detrusor muscle, urinary sphincter, bladder neck and the associated peripheral and central nervous system. According to the type of dysfunction, different clinical symptoms can arise. The Overactive Bladder Syndrome (OAB) is the most common disorder, and is defined as the presence of urinary urgency, usually accompanied by frequency and can occur with or without urinary incontinence. In contrast, lower urinary tract dysfunction (LUTD) can also result in the inability to evacuate urine. Insufficient contraction of the detrusor can lead to difficult elimination or urinary retention: the underactive bladder. Suffering from LUTD can be very bothersome and has significant effects on many aspects of an individuals' life, representing a particularly impactful health burden to quality of life and productivity.

Epidemiology of lower urinary tract dysfunctions

According to the EpiLUTS study, a large population-based, cross-sectional internet survey, conducted in the UK, Sweden, and the USA, OAB and other lower urinary tract symptoms are problems many people suffer from. In the USA, 31,588 respondents completed the survey, making EpiLUTS one of the largest population-based epidemiological studies specifically focusing on OAB., ${ }^{1,2} \mathrm{De}-$ fined by the presence of urinary urgency of at least 'sometimes' and/or urge-urinary incontinence, $43 \%$ of female and $27 \%$ of male respondents reported OAB. With a more restrictive definition of at least 'often', the prevalence of OAB was $33 \%$ for women and $16 \%$ for men.

For the OAB Physical and Occupational Limitations (OAB-POLL) study 10,000 US adults were recruited to complete an internet survey regarding their OAB symptoms, demographic and clinical characteristics, and physical activity 
data, in order to estimate the prevalence of OAB and LUTS and the effects of $\mathrm{OAB}$ on work productivity and physical functioning. ${ }^{3}$ The authors defined $\mathrm{OAB}$ similarly to the EpiLUTS study, as urgency at least 'sometimes' and/or the presence of urge urinary incontinence. Based on this definition, the overall prevalence of $\mathrm{OAB}$ in the total US population was $23.3 \%$, with women reporting $\mathrm{OAB}$ almost twice as frequently as men (30.0 vs. $16.4 \%$, respectively).

\section{Socio-economic relevance}

Individuals with $O A B$ report significant impairment to overall quality of life: $\mathrm{OAB}$ interfered with daily activities, including symptoms that caused them to stay at home, decreased physical activity because of $O A B$ and even gaining weight because of an inability to exercise. Women with $O A B$ were also significantly more likely than those without $\mathrm{OAB}$ to report disturbed sleep, decreased self-esteem, decreased sexuality, and feelings of overall declining health. Findings from the NOBLE study also demonstrated significant effects of OAB on health-related quality of life as measured by validate surveys. ${ }^{4}$

From a patient's perspective, the costs associated with OAB tends to be for routine care, such as incontinence pads, diapers, and laundry, generally reflecting the burden of urinary incontinence.

Estimates of the individual and societal costs for the management of OAB continue to rise. In 2007, average annual per capita costs of OAB in the United States were \$1925 (\$1433 in direct medical, \$66 in direct nonmedical, and \$426 in indirect costs). Applying these costs to the 34 million people in the United States with OAB results in total national costs of $\$ 65.9$ billion. Average annual costs in 2015 and 2020 would be $\$ 76.2$ billion and $\$ 82.6$ billion, respectively. ${ }^{5}$

People with symptoms of OAB often delay seeking treatment until increased symptom severity or bother appear. An important aspect of $O A B$ is that it remains difficult to treat effectively. Pharmacotherapy is generally effective based on individual clinical trials but often only modestly superior to placebo.

In chapter 8 (general discussion) we describe that SNM has been shown to be both more effective and cost saving, or at least acceptably cost-effective compared to other therapies (PTNS or BoNT-A).

Autiero et al. compared SNM with optimal medical therapy, BoNT-A and PTNS in the UK. At 5 years, SNM was more effective and less costly than PTNS. Compared with ongoing medical therapy at 10 years, SNM was more costly and more effective, and compared with BoNT-A, SNM with PNE was less costly and more effective, and SNM with TLP was more costly and more effective. ${ }^{6}$ The costs for SNM mainly involve device acquisition and implantation. All other 
treatments involve on-going drug costs and physician visits for maintenance treatment.

Recently the results of the ROSETTA trail were presented. In this multicenter open-label randomized trial to assess whether BoNT-A is superior to SNM 369 women with refractory urgency urinary incontinence were treated, and 364 were available for the primary outcome analyses. The BoNT-A group reported significantly greater mean reduction in incontinence episodes per day compared to the SNM group. The BoNT-A group was significantly more likely to experience complete resolution of urgency incontinence, report greater improvements in overactive bladder symptom bother. Urinary tract infections were higher in the BoNT-A group (35\% vs.11\%, p<0.001). Self-catheterization was required in $8 \%$ and $2 \%$ of the BoNT-A group at one and six months, respectively and neuromodulation device revisions/removals occurred in $3 \%$ ?

It has to be notified that most studies regarding cost effectiveness for SNM received financial support by the manufacturer of SNM devices, which possibly introduces a bias.

\section{Target population}

The results of this manuscript are relevant to patients with symptoms of lower urinary tract dysfunction such as frequency, urgency-incontinence or urinary retention. Some of our results are also important for patients who suffer from the combination of urinary tract dysfunctions as well as fecal problems (incontinence and/or constipation). For example patients can be better informed on what results and possible complications to expect and patients can benefit from longer battery life.

Furthermore our study data can be relevant for medical device corporations who want to develop less costly devices then the ones that are used today. These devices should be small, rechargeable and MRI compatible.

\section{Products}

Although no new products have been developed with the results of this thesis directly, we showed that changes made in the way of implantation are safe and effective (chapter 4). We also found that patients prefer to have control over the devices used in SNM (chapter 5), the possibilities for self control can be expanded in future designs. The size of the implantable pulse generator is already reduced for better patient comfort, and another company introduced a smaller and rechargeable device for more patient comfort and efficacy. 
Innovation and future

In our view, the improvement of SNM should be targeted at:

- Increase in treatment efficacy

- Increase of patient comfort

- Reduction of adverse events and re-interventions

We estimate this can be reached by better patient selection and therefore a good test phase is most important. The test phase is preferably performed by a tined lead test phase while this gives more reliable results than testing with a PNE.

We showed that patients who are dissatisfied with BoNTA or when BoNTA treatment fails can be treated successfully with SNM.

Further improvement of patient comfort can be reached by using on-demand stimulation, with the positive side effect of more patient controlled therapy, or by conditional stimulation as we explained in chapter 8 .

For patients with urinary as well as fecal problems we advise a combined work-up in a center were treatment options such as SNM, PTNS and BoNTA can be used and treatments for functional problems of the lower urinary tract and of the bowel tract can be combined for more (cost) effectiveness. 


\section{References}

1 Coyne KS, Sexton CC, Vats V, Thompson C, Kopp ZS, Milsom I. National community prevalence of overactive bladder in the United States stratified by sex and age. Urology 2011;77:10817.

2 Milsom I, Kaplan SA, Coyne KS, Sexton CC, Kopp ZS. Effect of bothersome overactive bladder symptoms on health-related quality of life, anxiety, depression, and treatment seeking in the United States: results from EpiLUTS. Urology 2012;80:90-6.

3 Coyne KS, Sexton CC, Bell JA, et al. The prevalence of lower urinary tract symptoms (LUTS) and overactive bladder (OAB) by racial/ethnic group and age: results from OAB-POLL. Neurourol Urodyn 2013;32:230-7.

4 Reynolds WS, Fowke J, Dmochowski R. The Burden of Overactive Bladder on US Public Health. Curr Bladder Dysfunct Rep 2016;11:8-13.

5 Ganz ML, Smalarz AM, Krupski TL, et al. Economic costs of overactive bladder in the United States. Urology 2010;75:526-32, 32.e1-18.

6 Autiero SW, Hallas N, Betts CD, Ockrim JL. The cost-effectiveness of sacral nerve stimulation (SNS) for the treatment of idiopathic medically refractory overactive bladder (wet) in the UK. BJU Int 2015;116:945-54.

7 Amundsen CL, Richter HE, Menefee SA, et al. OnabotulinumtoxinA vs Sacral Neuromodulation on Refractory Urgency Urinary Incontinence in Women: A Randomized Clinical Trial. Jama 2016;316:1366-74. 


\section{DANKWOORD}

Eindelijk! Dat is iets wat velen zullen denken nu mijn proefschrift een feit is.

Het heeft even geduurd en dat is natuurlijk voornamelijk aan mijzelf te wijten. Toch zijn er heel wat mensen in mijn omgeving die het gelukt is mij van mijn werk af te houden. Vakanties, vriendenweekendjes, autobeurzen, kroegbezoek, noem maar op. Ik heb er erg van genoten, maar helaas is dit proefschrift er niet sneller door afgerond. Diegenen die zich hieraan schuldig hebben gemaakt: bedankt voor deze momenten van ontspanning!

Een aantal mensen die me wel continu achtervolgd en geholpen hebben wil ik graag noemen.

Prof. dr. Ph.E.V.A. Van Kerrebroeck, promotor, beste Philip, je hebt me ooit aangenomen als onderzoeker waarmee mijn urologische carrière is begonnen. Inmiddels ben ik uroloog en ervan overtuigd dat dit promotietraject een goede investering is geweest. Dank voor je betrokken begeleiding, de introductie in de wetenschap, je aanwijzingen en je inzichten. Je bent in de loop van de jaren een van de mensen geweest die mij continu gestimuleerd heeft dit promotietraject af te ronden. Ik waardeer je vertrouwen.

Prof. dr. G.A. van Koeveringe, promotor, beste Gommert, ik ben blij dat jij me ook hebt willen helpen dit proefschrift af te ronden. Het is prettig om met je samen te werken en je ontwikkeling mee te hebben kunnen maken van uroloog tot hoofd van de afdeling. Jouw inzichten en kennis van de functionele urologie zijn van grote waarde.

Dr. E.H.J. Weil, copromotor, beste Ernest(o), vooral in de beginjaren was jij voor mij mijn meest directe begeleider. Van jou heb ik veel klinische zaken geleerd zoals de implantatie van neuromodulatie onder lokale anesthesie. Daarnaast heb je ontelbaar veel tips gegeven over het reilen en zeilen van het leven binnen maar zeker ook buiten het ziekenhuis! Bedankt voor deze prettige en leerzame samenwerking.

Dr. T.A.T. Marcelissen, copromotor, beste Tom, jij bent als aanstormend ta- 
lent precies op het goede moment op mijn pad gekomen. Jij had je proeve van bekwaamheid al doorstaan en je zocht een nieuwe wetenschappelijke uitdaging en ik kon jouw hulp goed gebruiken. Tom, bedankt voor je bijdragen en jou wacht een mooie toekomst als uroloog en wetenschapper!

De leden van de manuscriptcommissie, prof. dr. H.W.M. Steinbusch (voorzitter), prof. dr. K. Everaert, prof. dr. M. Van Kleef, prof. dr. A.A.B. Lycklama à Nijeholt en prof. dr. A.A.M. Masclee dank voor het beoordelen van mijn proefschrift.

Dr. R. de Jongh, beste Rick, toen ik als onderzoeker begon zat jij al in het hok. We hebben samen leuke en leerzame jaren doorgebracht op deze 5 vierkante meters waarbij ik in ieder geval veel van je heb opgestoken. Ik ben blij dat jij als paranimf en vriend aanwezig bent!

Prof. dr. M.A.T.M. van Vugt, beste Marcel, zonder twijfel was jij altijd al de echte wetenschapper van ons beiden. Met genoegen denk ik terug aan onze reizen die alle perfecte ingrediënten hadden. Dank dat je mij vanuit de campingstoel zo af en toe nog eens wat hebt bijgespijkerd over wetenschap, geneeskunde en Unox hamburgers. Super dat je er als paranimf bij kunt zijn.

Mijn collega onderzoekers Wout Scheepens en Anco van Voskuilen, het was prettig dat jullie al voorbereidend werk gedaan hadden waardoor ik niet het wiel opnieuw heb hoeven uitvinden. Wout, erg leuk dat je er bij kunt zijn als lid van de corona. En degenen die na mij gekomen zijn: Randall Leong, Tom Marcelissen, Martijn Smits en Jamie Drossaerts. Een deel van jullie heeft mij al ingehaald, Jamie jij bent er ook bijna! Bedankt voor de samenwerking.

Jarno Melenhorst, als collega onderzoeker van de heelkunde zaten we lang in hetzelfde schuitje. Wat een geluk dat ik in het zuiden jou als wijze uit het oosten tegenkwam. We vormden een goed team in de kliniek maar ook deelden we vaak dezelfde fantasieën over opgevoerde Saabs en saaie Volvo's. Een ideale combinatie!

Victor Zambon, jij hebt mij ooit als coassistent enthousiast gemaakt voor de urologie. Dankzij jou kon ik ook de volgende stappen zetten in de goede richting en kwam er contact met de urologen in Maastricht. Bedankt voor jouw enthousiasme en hulp.

Na mijn eerste jaren als onderzoeker moest ik ook nog opgeleid worden tot uroloog. Van de onderzoeker moest eerst een dokter gemaakt worden. Hiervoor ben ik de meeste dank verschuldigd aan mijn collega's uit het toenmalige Atrium MC: assistenten chirurgie en urologie, chirurgen en urologen bedankt.

Mijn speciale dank gaat uit naar dr. Rob Welten en dr. Karl Delaere: gelukkig 
mocht ik nog profiteren van jullie opleiding oude stijl.

Het laatste deel van mijn opleiding deed ik in het Maastricht UMC+ en hier kon ik mijn onderzoek weer oppakken. Dit was alleen maar mogelijk dankzij de leuke groep assistenten. Bedankt hiervoor.

Naast mij klaar te stomen als uroloog ben ik door de stafleden ook vaak gestimuleerd dit proefschrift af te maken, waarvoor dank. Speciale dank voor Kees van de Beek als opleider.

Het afronden van een proefschrift en de transformatie naar een daadwerkelijk boekje is onmogelijk zonder goede ondersteuning. Hiervoor ben ik erg blij geweest met de hulp van het secretariaat urologie van het MUMC+ en het LZR. Vooral Nancy dank voor al je hulp en tips. Katy dank voor je correcties. Zeer veel dank ook voor Patrick Ketelaars die de lay-out van dit proefschrift heeft verzorgd.

Mijn schoonouders, Noud en Rita Ketelaars, bedankt voor jullie hulp, met name voor het vaak acuut inspringen als oppas zodat ik toch aan mijn proefschrift kon werken.

Een woord van dank voor mijn familie: Karin en Janneke, jullie ondersteuning in goede en slechte tijden is onmisbaar. Pap en Mam: bedankt voor alle steun en mogelijkheden die jullie mij altijd gegeven hebben.

Moedertje, dat jij dit niet meer mee kan maken doet nog iedere dag pijn. Goede herinneringen blijven en je nuchtere kijk op dit proefschrift is daar een van: 'Je bent er zelf aan begonnen, dus zorg maar dat 't af komt.'

Mijn laatste woord van dank is voor mijn meest kostbare verzameling: mijn vrouwen. Livia, Anna en Rosa, strikt genomen hebben jullie ook wat vertraging van dit hele verhaal veroorzaakt, maar dat is niet erg. Jullie inspireren, jullie laten me lachen, jullie zijn onmisbaar. Fabrizia: je bent een droomvrouw, jouw hulp is onmisbaar. Ti voglio bene! 


\section{LIJST VAN PUBLICATIES}

Oerlemans DJAJ. Medicamenteuze behandeling van LUTS, urologie actueel, jaargang 18, nr.2, September 2015.

Oerlemans DJAJ, Smits MA, Marcellissen T, Van Kerrebroeck PE, de Wachter SG. Sacral neuromodulation in patients with idiopathic overactive bladder after initial botulinum toxin therapy. J Urol. 2013 Dec;190(6):2148-52.

Van Voskuilen AC, Oerlemans DJAJ, Gielen N, Lansen-Koch SM, Weil EH, van Lankveld JJ, van den Hombergh U, Baeten CG, Van Kerrenbroeck PE. Sexual Response in Patients Treated with Sacral Neuromodulation for Lower Urinary Tract Symptoms or Fecal Incontinence. Urol Int. 2012;88(4):423-30

Lansen-Koch SM, Govaert B, Oerlemans DJAJ, Melenhorst J, Vles H, Cornips E, Weil EH, van Heurn E, Baeten CG, van Gemert WG. Sacral nerve modulation for defaecation and micturition disorders in patients with spina bifida. Colorectal Dis. 2012 Apr;14(4):508-14.

Oerlemans DJAJ, van Voskuilen AC, Marcelissen T, Weil EH, de Bie RA, Van Kerrebroeck $\mathrm{PE}$. Is on-demand sacral neuromodulation in patients with $\mathrm{OAB}$ syndrome a feasible therapy regime? Neurourol Urodyn. 2011 Nov;30(8):1493-6.

Oerlemans DJAJ, Van Kerrebroeck PE. Sacral nerve stimulation for neuromodulation of the lower urinary tract. Neurourol Urodyn. 2008;27(1):28-33. Review.

Van Voskuilen AC, Oerlemans DJAJ, Weil EHJ, van den Hombergh U, Van Kerrebroeck PE. Medium-term experience of sacral neuromodulation by tined lead implantation. BJU Int. 2007 Jan;99(1):107-10. 
Van Kerrebroeck PE, Oerlemans DJAJ. Sacral Nerve Stimulation for Neuromodulation of the Lower Urinary Tract. European Urological Review, 2007;(1):88-9

Van Voskuilen AC, Oerlemans DJAJ, Weil EHJ, de Bie RA, Van Kerrebroeck PE. Long term results of neuromodulation by sacral nerve stimulation for lower urinary tract symptoms: a retrospective single center study. Eur Urol. 2006 Feb;49(2):366-72.

\section{Boekbijdragen}

Badlani G, Davila GW, Michel MC, Rosette JJMCH, (2009). Continence Current Concepts and Treatment Strategies. Heidelberg; Springer-Verlag. Chapter 17, Oerlemans DJAJ, van Kerrebroeck PE. Sacral nerve stimulation for neuromodulation of the lower urinary tract. 


\section{CURRIULUM VITAE}

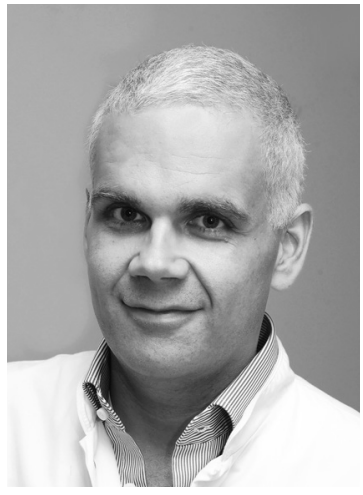

Dennis Oerlemans is geboren op 20 maart 1977 te 's-Hertogenbosch. In 1995 behaalde hij zijn diploma aan Gymnasium Beekvliet te Sint Michielsgestel. Hij studeerde gezondheidswetenschappen aan de Universiteit Maastricht (1996-1998) en in 1998 is hij gestart met geneeskunde aan de Universiteit Maastricht.

Zijn enthousiasme voor de urologie werd gewekt en zijn eerste ANIOS functie vond hij in 2004 in het Laurentius ziekenhuis Roermond. In 2005 startte hij als onderzoeker in het academisch ziekenhuis Maastricht. Het onderzoek naar de behandeling van chronische blaasklachten met behulp van sacrale neuromodulatie werd verricht onder begeleiding van prof. dr. Ph.E.V.A. Van Kerrebroeck en prof. dr. G.A. van Koeveringe (promotores) en dr. E.H.J. Weil en dr. T.A.T. Marcelissen (co-promotores). De opleiding tot uroloog begon in 2008 met de vooropleiding algemene heelkunde in het toenmalige Atrium MC te Heerlen met als opleider dr. R.J.Th.J. Welten. Het perifere urologische deel van zijn opleiding deed hij in hetzelfde ziekenhuis onder supervisie van dr. K.P.J. Delaere. Hij heeft zijn opleiding afgerond in het Maastricht UMC+ met drs. C. van de Beek als opleider.

$\mathrm{Na}$ zijn opleiding is hij gaan werken als uroloog in het Laurentius ziekenhuis Roermond met nevenbetrekkingen in het Maastricht UMC+ en waarneem periodes in het St-Anna Ziekenhuis en het VieCuri Medisch Centrum.

Dennis is gelukkig getrouwd met Fabrizia Ketelaars (2009) en samen hebben ze drie prachtige dochters: Livia (2011), Anna en Rosa (2013). 


\section{LIST OF ABBREVIATIONS}

$\begin{array}{ll}\text { BoNT-A } & \text { onabotulinum toxin-A } \\ \text { CISC } & \text { clean intermittent self catheterization } \\ \text { FI } & \text { fecal incontinence } \\ \text { INS } & \text { implantable neuro stimulator } \\ \text { IPG } & \text { internal pulse generator } \\ \text { LUTD } & \text { lower urinary tract dysfunction } \\ \text { OAB } & \text { overactive bladder (syndrome) } \\ \text { PNE } & \text { percutaneous nerve evaluation } \\ \text { PS } & \text { pudendal (nerve) stimulation } \\ \text { PTNS } & \text { percutaneous tibial nerve stimulation } \\ \text { SNM } & \text { sacral neuromodulation } \\ \text { SNS } & \text { sacral nerve stimulation } \\ \text { S3 } & \text { third sacral root } \\ \text { TENS } & \text { transcutaneous electrical nerve stimulation } \\ \text { TLP } & \text { tined lead procedure } \\ \text { UF } & \text { urgency frequency } \\ \text { UI } & \text { urinary incontinence } \\ \text { UR } & \text { (non obstructive) urinary retention }\end{array}$




\section{THESIS DEFENSES FROM MHENS}

MHeNs - School for Mental Health and Neuroscience.

2013

- $\quad$ Rob Havermans: Bipolar disorder in daily life; Mood and cortisol responses to naturally occurring events. Supervisor: prof. dr. M. de Vries; Co-Supervisor: Dr. N. Nicolson.

- Véronique Moers-Hornikx: Deep brain stimulation and the cerebellum. Supervisors: prof. dr. J. Vles/prof. dr. Y. Temel; Co-Supervisor: dr. G. Hoogland.

- Nicole Veldhorst-Janssen: Intranasal delivery of rapid acting drugs. Supervisors: prof. dr. M. Marcus/prof. dr. C. Neef; Co-Supervisor: dr. P.H. van der Kuy.

- Stéphanie Knippenberg: Vitamin D and Multiple Sclerosis: immunological and clinical outcome. Supervisor: prof. dr. J. Cohen-Tervaert; Co-Supervisors: dr. J. Damoiseaux/dr. Y. Bols.

- Erik D. Gommer: Dynamic Cerebral Autoregulation: from methodology towards clinical application. Supervisors: prof. dr. W.H. Mess/prof. dr. R.B. Panerai, UK; Co-Supervisor: dr. ir. J.P.H. Reulen.

- Olga A.H. Reneerkens: Can PDE inhibition improve cognition? Translational insights. Supervisor: prof. dr. H.W.M. Steinbusch; Co-Supervisor: Dr. J. Prickaerts;.

- $\quad$ Lyzel S. Elias-Sonnenschein: Clinical and biomarker correlates of genetic risk factors for Alzheimer's disease. Supervisor: prof. dr. F.R.J. Verhey; Co-Supervisor: dr. P.J. Visser.

- Diego F. Mastroeni: Epigenetic Dysregulation and the Pathophysiology of of Alzheimer's Disease. Supervisors: prof. dr. H.W.M. Steinbusch/prof. dr. P.D. Coleman, Sun City, Arizona; Co-Supervisors: dr. B.P.F. Rutten/dr. D.L.A. van den Hove.

- Leonidas Chouliaras: Epigenetic Regulation in Aging and Alzheimer's disease: A translational perspective. Supervisor: prof. dr. H.W.M. Steinbusch; Co-Supervisors: dr. B.P.F. Rutten/dr. D.L.A. van den Hove.

- Liesbeth Knaepen: Perinatal events and altered pain sensitivity in later life. Supervisors: prof. dr. E.A.J. Joosten/prof. dr. D. Tibboel, EUR; Co-Supervisor: dr. J. Patijn.

- Marisela Martinez-Claros: Hippocampal plasticity and corticosterone: From dendrites to behaviour. Supervisor: prof. dr. H.W.M. Steinbusch; Co-Supervisors: dr. J.L. Pawluski/dr. J. Prickaerts.

- Marcus D. Lancé: A circle of improvement in bleeding management: from laboratory to clinic and back. Supervisors: prof. dr. M.A.E. Marcu/prof. dr. J.W.M. Heemskerk; Co-Supervisor: dr. Y.M.C. Henskens.

- Hilde Braakman: Imaging the brain; neuronal correlates of cognitive impairment in children with frontal lobe epilepsy. Supervisors: prof. dr. A.P. Aldenkamp/prof. dr. J.S.H. Vles; Co-Supervisors: dr. ir. W.H. Backes/dr. P.A.M. Hofman.

- Willem H. van Zwam: Aneurysmal subarachnoid hemorrhage: imaging strategies and cost-effectiveness aspects in diagnostic work-up and post-therapeutic follow-up. Supervisors: prof. dr. J.T. Wilmink/prof. dr. J.E. Wildberger; Co-Supervisor: dr. P.A.M. Hofman. 
- Klara De Cort: The Pathogenesis of Panic Disorder. Supervisors: prof. dr. I. Myin-Germeys/prof. dr. E.J.L. Griez; Co-Supervisors: dr. K.R.J. Schruers/dr. I. Van Diest, Leuven.

- Kim van Wijck: Mind the Gap; experimental studies on splanchnic hyperfusion and gastrointestinal integrity loss in man. Supervisors: prof. dr. W.A. Buurman/prof. dr. C.H.C. Dejong; Co-Supervisor: dr. K. Lenaerts.

- Yvette Roke: Antipsychotic-induced hyperprolactinemia in children and adolescents with mainly autism spectrum disorders. Prevalence, symptoms, clinical consequences and genetic risk factors. Supervisors: prof. dr. P.N. van Harten/prof. dr. J.K. Buitelaar (RUN); Co-Supervisor: dr. A. Boot (UMCG).

- Fleur Goezinne: Retinal detachment surgery: pre and postoperative prognostic factors. Supervisors: prof. dr. F. Hendrikse/prof.dr. C.A.B. Webers; Co-Supervisor: dr. E.C. La Heij (Amsterdam).

- Ralph L.J.G. Maassen: The Merits of Videolaryngoscopy during Glottic Visualisation for Endotracheal Intubation. Supervisors: prof. dr. M. Marcus/prof. dr. A. van Zundert (University of Queensland).

- Maria J. de Sousa Guerreiro: The role of sensory modality in age-related distraction. Supervisor: prof. dr. C.M. van Heugten; Co-Supervisor: dr. P.W.M. van Gerven.

- Ine Rayen: Effects of developmental fluoxetine exposure on neurobehavioral outcomes. Supervisor: prof. dr. H.W.M. Steinbusch; Co-Supervisors: dr. J.L. Pawluski/dr. T.D. Charlier (Ohio University, USA).

- Nynke M.G. Bodde: Psychogenic non-epileptic seizures; a separate disorder or part of a continuum? Supervisors: prof. dr. R. van Oostenbrugge/prof. dr. K. Vonck (UZ Gent); Co-Supervisors: dr. R. Lazeron/dr. A. de Louw (Epilepsiecentrum Kempenhaeghe, Heeze).

- Alejandro M. Gomez: Novel strategies for making myasthenia less gravis: targeting plasma cells and the neuromuscular junction. Supervisor: prof. dr. M.H. De Baets; Co-Supervisors: dr. M. Losen/ dr. P. Martinez-Martinez.

- Mohammad S. Rahnama'i: Prostaglandins and Phosphodiesterases in the Urinary Bladder Wall. Supervisors: prof. dr. Ph. Van Kerrebroeck/prof. dr. S. de Wachter (Universiteit Antwerpen); Co-Supervisor: dr. G. van Koeveringe.

- Mariken B. de Koning: Studying biomarkers in populations at genetic and clinical high risk for psychosis. Supervisors: Prof.dr. T. Amelsvoort / Prof.dr. J. Booij (AMC).

- Fabien Boulle: Epigenetic regulation of BDNF/TrkB signaling in the pathophysiology and treatment of mood disorders. Supervisors: prof. dr. H.W.M. Steinbusch/prof. dr. L. Lanfumey (Universiteit Parijs); Co-Supervisors: dr. D. van den Hove/Dr. G. Kenis.

2014

- Iris Nowak-Maes: Tinnitus; assessment of quality of life $\mathcal{E}$ cost-effectiveness. Supervisors: prof. dr. M. Peters/prof. dr. B. Kremer; Co-Supervisors: dr. M. Joore/dr. L. Anteunis.

- Marjolein Huijts: Cognitive function in patients with cerebral small vessel disease. Supervisor: prof. dr. R.J. van Oostenbrugge; Co-Supervisors: dr. A.A. Duits/dr. J. Staals.

- Markus Gantert: Fetal inflammatory injury as origin of long term disease: Lessons from animal models. Supervisors: prof. dr. B. Kramer/prof. dr. L. Zimmermann; Co-Supervisor: dr. A. Gavilanes.

- Elke Kuypers: Fetal development after antenatal exposures: Chorioamnionitis and maternal glucocorticoids. Supervisors: prof. dr. B.W. Kramer/prof. dr. H.W. Steinbusch/prof. dr. Suhas G. Kallapur (University of Cincinnati, Ohio, USA).

- Pieter Kubben: Ultra low-field strength intraoperative MRI for Glioblastoma Surgery. Supervisor: prof. dr. J.J. van Overbeeke; Co-Supervisor: dr. H. van Santbrink. 
- Laura Baijens: Surface electrical stimulation of the neck for oropharyngeal dysphagia in Parkinson's disease: therapeutic aspects and reliability of measurement. Supervisor: prof. dr. B. Kremer; Co-Supervisor: dr. R. Speyer, Townsville.

- Janneke Hoeijmakers: Small fiber neuropathy and sodium channels; a paradigm shift. Supervisor: prof. dr. R.J. van Oostenbrugge; Co-Supervisors: dr. C.G. Faber/dr. I.S.J. Merkies.

- Stephanie Vos: The Role of biomarkers in preclinical and prodromal Alzheimer's disease. Supervisor: prof. dr. F.R. Verhey; Co-Supervisor: dr. P.J. Visser.

- Muriël Doors: The Value of Optical Coherence Tomography in Anterior Segment Surgery. Supervisors: prof. dr. R.M. Nuijts/prof. dr. C.A. Webers; Co-Supervisor: Dr. T.T.J.M. Berendschot.

- Anneke Maas: Sleep problems in individuals with genetic disorders associated with intellectual disability. Supervisors: prof. dr. I. Curfs/prof.dr. R. Didden.

- Sebastiaan van Gorp: Translational research on spinal cord injury and cell-based therapies; a focus on pain and sensorimotor disturbances. Supervisors: Prof.dr. B. Joosten / Prof.dr. M. van Kleef; Co-Supervisors: Dr. J. Patijn /Dr. R. Deumens, KU Leuven.

- Andrea Sannia: High risk newborns and brain biochemical monitoring. Supervisor: prof. dr. J.S.H. Vles; Co-Supervisors: dr. D. Gazzolo, Alessandria, Italy/dr. A.W.D. Gavilanes.

- Julie A.D.A. Dela Cruz: Dopamine mechanisms in learning and memory: Evidence from rodent studies. Supervisors: prof. dr. H.W.M. Steinbusch/prof. dr. R.J. Bodnar, New York; Co-Supervisor: dr. B.P.F. Rutten.

- René Besseling: Brain wiring and neuronal dynamics; advances in MR imaging of focal epilepsy. Supervisors: prof. dr. A.P. Aldenkamp/prof. dr. ir. W.H. Backes; Co-Supervisor: dr. J.F.A. Jansen.

- Maria Quint-Fens: Long-term care after stroke; development and evaluation of a long-term intervention in primary care. Supervisors: prof. dr. J.F.M. Metsemakers/prof. dr. C.M. van Heugten/prof. dr. M. Limburg, Almere; Co-Supervisor: dr. G.H.M.I. Beusmans.

- Veronique Moulaert: Life after survival of a cardiac arrest; the heart of the matter. Supervisors: prof. dr. J.A. Verbunt/prof. dr. C.M. van Heugten/prof. dr. D.T. Wade, Oxford, UK.

- Feikje Smeets: The hallucinatory-delusional state: a crucial connection in the psychosis symptom network. Supervisor: prof. dr. J. van Os; Co-Supervisor: dr. T. Lataster.

- Lies Clerx: Alzheimer's disease through the MR-eye; novel diagnostic markers and the road to clinical implementation. Supervisor: prof. dr. F. Verhey; Co-Supervisors: dr. P.J. Visser/P. Aalten.

- Sonny Tan: The subthalamic nucleus in Parkinson's disease. Supervisors: prof. dr. Y. Temel/prof. dr. H.W.M. Steinbusch/prof. dr. T. Sharp, Oxford, UK/prof. dr. V. Visser-Vandewalle, Koln.

- Koen van Boxem: The use of pulsed radiofrequency in the management of chronic lumbosacral radicular pain. Supervisors: prof. dr. M. van Kleef/prof. dr. E.A.J. Joosten; Co-Supervisor: assoc. prof. dr. J. van Zundert.

- Jérôme Waterval: Hyperostosis cranialis interna. Supervisors: prof. dr. J.J. Manni/prof. dr. R.J. Stokroos.

- Sylvie Kolfschoten-van der Kruijs: Psychogenic non-epileptic seizures; the identification of neurophysiological correlates. Supervisors: prof. dr. A.P. Aldenkamp/prof. dr. K.E.J. Vonck, Universiteit Gent; Co-Supervisors: dr. J.F.A. Jansen/dr. R.H.C. Lazeron, Kempenhaeghe.

- Wouter Pluijms: Spinal cord stimulation and pain relief in painful diabetic: polyneuropathy, a translational approach. Supervisors: prof. dr. M. van Kleef/prof. dr. E.A. Joosten; Co-supervisor: dr. C.G. Faber.

- Ron Handels: Health technology assessment of diagnostic strategies for Alzheimer's disease. Supervisors: prof. dr. F.R.J. Verhey/prof. dr. J.L. Severens (EUR); Co-Supervisor: dr. M.A. Joore/dr. C.A.G. Wolfs.

- Evelyn Peelen: Regulatory T cells in the pathogenesis of Multiple Sclerosis: potential targets for vitamin D therapy. Supervisors: prof. dr. R.M.M. Hupperts/prof. dr. J.W. Cohen Tervaert; Co-Supervisor: dr. J.G.M.C. Damoiseaux/dr. M.M.G.L.Thewissen, Diepenbeek. 
- Reint Jellema: Cell-based therapy for hypoxic-ischemic injury in the preterm brain. Supervisors: prof. dr. B.W.W. Kramer/prof. dr. H.W.M. Steinbusch; Co-Supervisor: dr. W.T.V. Germeraad/dr. P. Andriessen, Veldhoven.

- Maria Wertli: Prognosis of Chronic Clinical Pain Conditions: The Example of Complex Regional Pain Syndrome 1 and Low Back Pain. Supervisors: prof. dr. M. van Kleef; Co-Supervisor: dr. F. Brunner, Zürich/dr. R. Perez, VUmc.

- Dagmar Zeef: An experimental model of Huntington's disease: Validation \& Stimulation. Supervisors: prof. dr. Y. Temel/prof. dr. H.W.M. Steinbusch; Co-supervisor: dr. A. Jahanshahi.

- Jeroen Decoster: Breaking Down Schizophrenia into phenes, genes and environment. Supervisors: prof. dr. I. Myin-Germeys/prof. dr. M. De Hert, KU Leuven; Co-Supervisor: dr. R. van Winkel.

- Eaja Anindya Sekhar Mukherjee: Fetal Alcohol Spectrum Disorders: exploring prevention and management. Supervisor: prof. dr. L.M.G. Curfs; Co-Supervisor: prof. S. Hollins, St. George's University of London, UK.

- Catherine van Zelst: Inside out; On stereotype awareness, childhood trauma and stigma in psychosis. Supervisors: prof. dr. Ph. Delespaul/prof. dr. J. van Os.

- Ibrahim Tolga Binbay: Extended Psychosis Phenotype in the Wider Social Environment. Supervisor: prof. dr. J. van Os; Co-Supervisor: dr. M. Drukker.

- Frank Van Dael: OCD matters in psychosis. Supervisors: prof. dr. J. van Os/prof. dr. I. Myin-Germeys.

- Pamela Kleikers: NOXious oxidative stress: from head toe too and back. Supervisors: prof. dr. H.H.H.W. Schmidt/prof. dr. H.W.M. Steinbusch; Co-Supervisor: dr. B. Janssen.

- José Luis Gerardo Nava: In vitro assay systems in the development of therapeutic interventions strategies for neuroprotection and repair. Supervisors: prof. dr. med. J. Weis/prof. dr. H.W.M. Steinbusch; Co-Supervisor: dr. G.A. Brook, RWTH Aachen.

- Eva Bollen: Cyclic nucleotide signaling and plasticity. Supervisors: prof. dr. H.W.M. Steinbusch/ prof. dr. R. D’Hooge, KU Leuven; Co-Supervisor: dr. J. Prickaerts.

- Jessica A. Hartmann: A good laugh and a long sleep; Insights from prospective and ambulatory assessments about the importance of positive affect and sleep in mental health. Supervisor: prof. dr. J. van Os; Co-Supervisors: C.J.P. Simons/dr. M. Wichers.

- Bart Ament: Frailty in old age; conceptualization and care innovations. Supervisors: prof. dr. G.I.J.M. Kempen/prof. dr. F.R.J. Verhey; Co-Supervisor: dr. M.E. de Vugt.

- Mayke Janssens: Exploring course and outcome across the psychosis-continuum. Supervisor: prof. dr. I. Myin-Germeys; Co-Supervisor: dr. T. Lataster.

- Dennis M.J. Hernau: Dopayours is not dopamine: genetic, environmental and pathological variations in dopaminergic stress processing. Supervisor: prof. dr. I. Myin-Germeys; Co-Supervisors: prof. dr. F.M. Mottaghy/sr. D. Collip.

- Ingrid M.H. Brands: The adaptation process after acquired brain injury Pieces of the puzzle. Supervisors: prof. dr. C.M. van Heugten/prof. dr. D.T. Wade, Oxford UK; Co-Supervisors: dr. S.Z. Stapert/dr. S. Köhler.

- Francesco Risso: Urinary and salivary S10oB monitoring in high risk infants. Supervisor: prof. dr. J.S.H. Vles; Co-Supervisors: dr. D. Gazzolo, Genoa,Italy/dr. A.W.D. Gavilanes.

- Alessandro Borghesi: Stem and Progenitor Cells in Preterm Infants: Role in the Pathogenesis and Potential for Therapy. Supervisor: prof. dr. L. Zimmermann; prof. dr. B. Kramer; Co-Supervisors: dr. D. Gazzolo, Genoa,Italy/dr. A.W.D. Gavilanes.

- Claudia Menne-Lothmann: Affect dynamics; A focus on genes, stress, and an opportunity for change. Supervisor: prof. dr. J. van Os; Co-Supervisors: dr. M. Wichers/dr. N. Jacobs. 
- Martine van Nierop: Surviving childhood new perspectives on the link between childhood trauma and psychosis. Supervisors: prof. dr. I. Myin-Germeys/prof. dr. J. van Os; Co-Supervisor: dr. R. van Winkel.

- Sylvia Klinkenberg: VNS in children; more than just seizure reduction. Supervisors: prof. dr. J. Vles/prof. dr. A. Aldenkamp; Co-Supervisor: dr. H. Majoie.

- Anouk Linssen: Considerations in designing an adult hearing screening programme. Supervisor: prof. dr. B. Kremer; Co-Supervisors: dr. L. Anteunis/dr. M. Joore.

- Janny Hof: Hearing loss in young children; challenges in assessment and intervention. Supervisors: Prof.dr. B. Kremer / Prof.dr. R. Stokroos / Prof.dr. P. van Dijk, RUG; Co-Supervisor: dr. L. Antheunis.

- Kimberly Cox-Limpens: Mechanisms of endogenous brain protection; Clues from the transcriptome. Supervisors: prof. dr. J. Vles/prof. dr. L. Zimmermann; Co-Supervisor: dr. A. Gavilanes.

- Els Vanhoutte: Peripheral Neuropathy outcome measures; Standardisation (PeriNomS) study part 2: Getting consensus. Supervisors: prof. dr. C. Faber/prof. dr. P. van Doorn; Co-Supervisor: dr. I. Merkies, Spaarne ziekenhuis Hoofddorp.

- Mayienne Bakkers: Small fibers, big troubles; diagnosis and implications of small fiber neuropathy. Supervisors: prof. dr. C. Faber/prof.dr. M. de Baets; Co-Supervisor: dr. I. Merkies, Spaarne ziekenhuis Hoofddorp.

- Ingrid Kramer: Zooming into the micro-level of experience: An approach for understanding and treating psychopathology. Supervisor: prof. dr. J. van Os; Co-Supervisors: dr. M. Wichers, UMC Groningen/dr. C. Simons.

- Esther Bouman: Risks and Benefits of Regional Anesthesia in the Perioperative Setting. Supervisors: prof. dr. M. van Kleef/prof. dr. M. Marcus, HMC, Qatar/prof. dr. E. Joosten; Co-Supervisor: dr. H. Gramke.

- Mark Janssen: Selective stimulation of the subthalamic nucleus in Parkinson's disease; dream or near future. Supervisors: prof. dr. Y. Temel/prof. dr. V. Visser-Vandewalle, Keulen/prof. dr. A. Benazzouz, Bordeax, France.

- Reina de Kinderen: Health Technology Assessment in Epilepsy; economic evaluations and preference studies. Supervisors: prof. dr. S. Evers/prof. dr. A. Aldenkamp; Co-Supervisor: dr. H. Majoie/dr. D. Postulart, GGZ O-Brabant.

- Saskia Ebus: Interictal epileptiform activity as a marker for clinical outcome. Supervisors: prof. dr. A. Aldenkamp/prof. dr. J. Arends, TUE/prof. dr. P. Boon, Universiteit Gent, België.

- Inge Knuts: Experimental and clinical studies into determinants of panic severity. Supervisor: prof. dr. I. Myin-Germeys; Co-Supervisor: dr. K. Schruers; Influencing panic.

- Nienke Tielemans: Proactive coping post stroke: The Restored4Stroke Self-Management study. Supervisors: prof. dr. C. van Heugten/prof. dr. J. Visser-Meily, UMC Utrecht; Co-Supervisor: dr. V. Schepers, UMC Utrecht.

- Tom van Zundert: Improvements Towards Safer Extraglottic Airway Devices. Supervisors: prof. dr. A.E.M. Marcus/prof. dr. W. Buhre/prof. dr. J.R. Brimacombe, Queensland, Australia/prof. dr. C.A. Hagberg.

- Tijmen van Assen: Anterior Cutaneous Nerve Entrapment Syndrome Epidemiology and surgical management. Supervisors: prof. dr. G.L. Beets/prof. dr. M. van Kleef/dr. R.M.H. Roumen/dr. M.R.M. Scheltinga, MMC Veldhoven.

- Rohit Shetty: Understanding the Clinical, Immunological and Genetic Molecular Mechanisms of Keratoconus. Supervisors: prof. dr. R.M.M.A. Nuijts/prof. dr. C.A.B. Webers.

- Christine van der Leeuw: Blood, bones and brains; peripheral biological endophenotypes and their structural cerebral correlates in psychotic disorder. Supervisor: prof. dr. J. van Os; Co-supervisor: dr. M. Marcelis. 
- Sanne Peeters: The Idle Mind Never Rests; functional brain connectivity across the psychosis continuum. Supervisor: prof. dr. J. van Os; Co-supervisor: dr. M. Marcelis.

- Nick van Goethem: 7 nicotinic acetylcholine receptors and memory processes: mechanistic and behavioral studies. Supervisor: prof. dr. H.W.M. Steinbusch; Co-supervisor: dr. J. Prickaerts.

- Nicole Leibold: A Breath offear; a translational approach into the mechanisms of panic. Supervisor: prof. dr. H.W.M. Steinbusch; Co-supervisors: dr. K.R.J. Schruers/dr. D.L.A. van den Hove.

- Renske Hamel: The course of mild cognitive impairment and the role of comorbidity. Supervisor: prof. dr. F.R.J. Verhey; Co-supervisors: dr. I.H.G.B. Ramakers/dr. P.J. Visser.

- Lucia Speth: Effects of botulinum toxin A injections and bimanual task-oriented therapy on hand functions and bimanual activities in unilateral Cerebral Palsy. Supervisors: prof. dr. J. Vles; prof. dr. R. Smeets; Co-supervisor: dr. Y. Janssen-Potten, Adelante Hoensbroek.

- Yuan Tian: The effects of Lutein on the inflammatory pathways in age-related macular degeneration $(A M D)$. Supervisors: prof. dr. C. Webers; prof. dr. A. Kijlstra, WUR; Co-supervisor: dr. M. Spreeuwenberg; dr. H. Tange.

- Peggy Spauwen: Cognition and Type 2 diabetes; the interplay of risk factors. Supervisors: prof. dr. F. Verhey; prof. dr. C. Stehouwer; Co-supervisor: dr. M. van Boxtel.

- Marc Hilhorst: Crescentic glomerulonephritis in ANCA associated vasculitis. Supervisors: prof. dr. J. Cohen-Tervaert; Co-supervisor: dr. P. van Paassen.

- Martin Gevonden: The odd one out: exploring the nature of the association between minority status and psychosis. Supervisors: prof. dr. J-P. Selten; prof. dr. J. Booij, Uva; prof. dr. I. Myin-Germeys.

- Bart Biallosterski: Structural and functional aspects of sensory-motor Interaction in the urinary bladder. Supervisors: prof. dr. Ph. Van Kerrebroeck; prof. dr. S. De Wachter, UvAntwerpen; Co-supervisors: dr. G. van Koeveringe; dr. M. Rahnama'i.

- Alexandra König: The use of information and communication technologies (ICT) for the assessment of patients with Alzheimer's Disease and related disorders. Supervisors: prof. dr. F. Verhey; prof. dr. Ph. Robert, Nice, Fr; Co-supervisors: dr. P. Aalten; dr. R. David, Nice. France.

- Michelene Chenault: Assessing Readiness for Hearing Rehabilitation. Supervisors: prof. dr. M.P.F. Berger; prof. dr. B. Kremer; Co-supervisor: dr. L.J.C. Anteunis.

- Anand Vinekar: Retinopathy of Prematurity. Recent advances in tele-medicine screening, risk factors and spectral domain optical coherence tomography imaging. Supervisor: prof. dr. C.A.B. Webers; Co-supervisor: dr. N.J. Bauer.

- Fleur van Dooren: Diabetes and Depression: exploring the Interface between Pathophysiological and Psychological factors. Supervisors: prof. dr. F.R.J. Verhey; prof. dr. J.K.L. Denollet, UvT; prof. dr. F. Pouwer, UvT; Co-supervisor: dr. M.T. Schram.

- Gabriëlla Pons van Dijk: Taekwondo and physical fitness components in middle-aged healthy volunteers; the Sekwondo study. Supervisors: prof. dr. J. Lodder; prof. dr. H. Kingma; Co-supervisor: dr. A.F. Lenssen.

- Yara Pujol López: Development and psychoneuroimmunological mechanisms in depression. Supervisor: prof. dr. H.W.M. Steinbusch; Co-supervisors: dr. G. Kenis; dr. D. van den Hove; dr. Aye Mu Myint, München.

- Romina Gentier: UBB+1; an important switch in the onset of Alzheimer's disease. Supervisors: prof. H. Steinbusch; prof. D. Hopkins; Co-supervisor: dr. F. van Leeuwen.

- Sanne Smeets: Insights into insight: studies on awareness of deficits after acquired brain injury. Supervisor: prof. C. van Heugten; prof. R. Ponds; Co-supervisor: dr. I. Winkens

- Kim Beerhorst: Bone disease in chronic epilepsy: fit for a fracture. Supervisor: prof. A. Aldenkamp; prof. R. van Oostenbrugge; Co-supervisor: dr. P. Verschuure.

- Alex Zwanenburg: Cerebral and cardiac signal monitoring in fetal sheep with hypoxic-ischemic encephalopathy. Supervisor: prof. T. Delhaas; prof. B. Kramer; Co-supervisors: dr. T. Wolfs; dr. P. Andriessen, MMC. 
- Ismail Sinan Guloksuz: Biological mechanisms of environmental stressors in psychiatry. Supervisor: prof. J. van Os; Co-supervisors: dr. B. Rutten; dr. M. Drukker.

- Seyed Ehsan Pishva MD: Environmental Epigenetics in mental health and illness. Supervisor: prof. dr. J. van Os; Co-supervisors: dr. B.P.F. Rutten; dr. G. Kenis.

- Ankie Hamaekers: Rescue ventilation using expiratory ventilation assistance; innovating while clutching at straws. Supervisors: prof. dr. W.F. Buhre; prof. dr. M. van Kleef.

- Rens Evers: 22q11.2 deletion syndrome: intelligence, psychopathology and neurochemistry at adult age. Supervisors: prof. dr. L.M.G. Curfs; prof. dr. T. v. Amelsvoort.

- Sarah-Anna Hescham: Novel insights towards memory restoration. Supervisor: prof. dr. Y. Temel; Co-supervisor: dr. A. Blokland; dr. A. Jahanshahi.

- João P. da Costa Alvares Viegas Nunes: Insulin receptor sensitization improves affective pathology in various mouse models. Supervisor: prof. dr. H.W.M. Steinbusch; Co-supervisors: dr. K-P. Lesch; dr. T. Strekalova; dr.B.H. Cline, Oxford.

- Yanny Ying-Yee Cheng: Clinical Outcomes After Innovative Lamellar Corneal Transplantation Surgery. Supervisor: prof. dr. R.M.M.A. Nuijts; Co-supervisor: dr. J.S.A.G. Schouten.

2016

- Oliver Gerlach: Parkinson's disease, deterioration during hospitalization. Supervisor: prof. dr. R. van Oostenbrugge; Co-supervisor: dr. W. Weber.

- Remo Arts: Intracochlear electrical stimulation to suppress tinnitus. Supervisor: prof. dr. R.J. Stokroos; Co-supervisor: dr. E.L.J. Georg.

- Mitchel van Eeden: The $€$ - Restore4stroke study: Economic evaluation of stroke care in the Netherlands. Supervisors: prof. dr.mr. S.M.A.A. Evers; prof. dr. C.M. v. Heugten; Co-supervisor: dr. G.A.P. van Mastrigt.

- Pim Klarenbeek: Blood pressure and cerebral small vessel disease. Supervisor: prof. dr. R.J. van Oostenbrugge; Co-supervisor: dr. J. Staals.

- Ramona Hohnen: Peripheral pharmacological targets to modify bladder contractility. Supervisor: prof. dr. Ph.E.V. van Kerrebroeck; Co-supervisors: dr. G.A. van Koeveringe; dr. M.A. Sahnama'i; Dr. C. Meriaux.

- Ersoy Kocabicak: Deep brain stimulation of the subthalamic nucleus: Clinical and scientific aspects. Supervisors: prof. dr. Y. Temel; prof. dr. K. van Overbeeke; Co-supervisor: dr. A. Jahanshahi.

- Sven Akkerman: Temporal aspects of cyclic messenger signaling in object recognition memory; a pharmalogical approach. Supervisor: prof. dr. H.W.M. Steinbusch; Co-supervisors: dr. J. Prickaerts; dr. A. Blokland.

- Anja Moonen: Emotion and Cognition in Parkinson's disease; etiology and neurobiological mechanisms. Supervisor: prof. dr. F.R.J. Verhey; Co-supervisor: dr. A.F.G. Leentjens.

- Anna Schüth: Three-dimensional bladder tissue morphology. Supervisors: prof. dr. G.A. van Koeveringe; prof. dr. M. v. Zandvoort, Aachen; prof. dr. Ph. V. Kerrebroeck.

- Elisabeth van der Ven: Ethnic minority position as risk indicator for autism-spectrum and psychotic disorders. Supervisors: prof. dr. J.P. Selten; prof. dr. J. van Os.

- Zuzana Kasanova: Environmental reactivity for better or worse; The impact of stress and reward on neurochemistry, affect and behavior across the psychosis continuum. Supervisor: prof. dr. I. Myin-Germeys, KU Leuven/UM; Co-supervisor: dr. D. Collip.

- Danielle Lambrechts: Ketogenic diet therapies; treatment for children and adults with refractory epilepsy. Supervisors: Prof.dr. H.J.M. Majoie; Prof.dr. J.S.H. Vles; prof. dr. A.P. Aldenkamp; Co-supervisor: dr. A.J.A. de Louw, Kempenhaghe, Heeze.

- Frank van Bussel: Advanced MRI in diabetes; cerebral biomarkers of cognitive decrements. Supervisors: Prof.dr.ir. W.H. Backes; Prof.dr. P.A.M. Hofman; Co-supervisor: dr. J.F.A. Jansen. 
- Lisa Schönfeldt: Neurostimulation to treat brain injury? Supervisors: prof. dr. Y. Temel; prof. dr. S. Hendrikx, Hasselt; Co-supervisor: dr. A. Jahanshahi.

- Rianne Geerlings: Transition in patients with childhood-onset epilepsy; a long way to adulthood. Supervisor: prof. dr. A.P. Aldenkamp; Co-supervisors: dr. A.J.A. de Louw, dr. L.M.C. Gottmer, Kempenhaeghe.

- Nele Claes: B cells as multifactorial players in multiple sclerosis pathogenesis: insights from therapeutics. Supervisors: prof. dr. V. Somers, Hasselt; prof. dr. R. Hupperts; Co-supervisors: prof. dr. P. Stinissen, dr. J. Fraussen, Hasselt.

- Olaf Schijns: Epilepsy surgery and biomarkers from history to molecular imaging. Supervisors: prof. dr. J.J. van Overbeeke; prof. dr. H. Clustermann, Aachen; Co-supervisors: dr. G. Hoogland; dr. M.J.P. v. Kroonenburgh.

- Lizzy Boots: Balanced and Prepared; development and evaluation of a supportive e-health intervention for caregivers of people with early-stage dementia. Supervisors: prof. dr. F.R.J. Verhey; prof. dr. G.I.J.M. Kempen; Co-supervisor: dr. M.E. de Vugt.

- Wouter Donders: Towards patient-specific (cerebro-) vascular model applications. Supervisors: prof. dr. T. Delhaas; prof. dr. ir. F.N. van de Vosse, TUE; Co-supervisor: dr. ir. W. Huberts.

- Sizzle Vanterpool: The implications of intrauterine invasion by microbes for placental Pathology and the occurrence of adverse pregnancy outcomes. Supervisor: prof. dr. B.W. Kramer. Co-supervisors: dr. J.V. Been, Erasmus MC Rotterdam, dr. U von Rango. 
